\title{
Análise de Componentes Esparsos Locais com Aplicações em Ressonância Magnética Funcional
}

\author{
Gilson Vieira
}

TESE APRESENTADA

$\mathrm{AO}$

Instituto DE MATEMÁtica e EstatísticA

DA

UniversidAde DE SÃo PAUlo

PARA

OBTENÇÃO DO TÍTULO

$\mathrm{DE}$

DOUTOR EM CIÊNCIAS

Programa: Bioinformática

Orientador: Prof. Dr. Luiz Antonio Baccalá

Coorientador: Prof. Dr. Edson Amaro Jr.

Durante o desenvolvimento deste trabalho o autor recebeu auxílio financeiro da CAPES

São Paulo, Agosto de 2015 


\section{Análise de Componentes Esparsos Locais com Aplicação em Ressonância Magnética Funcional}

Esta versão da tese contém as correções e alterações sugeridas pela Comissão Julgadora durante a defesa da versão original do trabalho, realizada em 13/10/2015. Uma cópia da versão original está disponível no Instituto de Matemática e Estatística da Universidade de São Paulo.

Comissão Julgadora:

- Prof. Dr. Luis Antonio Baccalá (orientador) - POLI-USP

- Prof. Dr. André Fujita - IME-USP

- Prof. Dr. José Roberto Castilho Piqueira - POLI-USP

- Prof. Dr. Claudinei Eduardo Biazoli Junior - UFABC

- Prof. Dr. Ellison Fernando Cardoso - FM-USP 


\section{Agradecimentos}

Ao meu querido filho Vincent por me tornar uma pessoa melhor a cada dia. 


\section{Resumo}

Vieira, G. Análise de Componentes Esparsos Locais com Aplicação em Ressonância Magnética Funcional. 2015. 104 f. Tese (Doutorado) - Instituto de Matemática e Estatística, Universidade de São Paulo, São Paulo, 2015.

Esta tese apresenta um novo método para analisar dados de ressonância magnética funcional (FMRI) durante o estado de repouso denominado Análise de Componentes Esparsos Locais (LSCA). A LSCA é uma especialização da Análise de Componentes Esparsos (SCA) que leva em consideração a informação espacial dos dados para reconstruir a informação temporal de fontes bem localizadas, ou seja, fontes que representam a atividade de regiões corticais conectadas. Este estudo contém dados de simulação e dados reais. Os dados simulados foram preparados para avaliar a LSCA em diferentes cenários. Em um primeiro cenário, a LSCA é comparada com a Análise de Componentes Principais (PCA) em relação a capacidade de detectar fontes locais sob ruído branco e gaussiano. Em seguida, a LSCA é comparada com o algoritmo de Maximização da Expectativa (EM) no quesito detecção de fontes dinâmicas locais. Os dados reais foram coletados para fins comparativos e ilustrativos. Imagens de FMRI de onze voluntários sadios foram adquiridas utilizando um equipamento de ressonância magnética de $3 \mathrm{~T}$ durante um protocolo de estado de repouso. As imagens foram pré-processadas e analisadas por dois métodos: a LSCA e a Análise de Componentes Independentes (ICA). Os componentes identificados pela LSCA foram comparados com componentes comumente reportados na literatura utilizando a ICA. Além da comparação direta com a ICA, a LSCA foi aplicada com o propósito único de caracterizar a dinâmica das redes de estado de repouso. Resultados simulados mostram que a LSCA é apropriada para identificar fontes esparsas locais. Em dados de FMRI no estado de repouso, a LSCA é capaz de identificar as mesmas fontes que são identificadas pela ICA, permitindo uma análise mais detalhada das relações entre regiões dentro de e entre componentes e sugerindo que muitos componentes identificados pela ICA em FMRI durante o estado de repouso representam um conjunto de componentes esparsos locais. Utilizando a LSCA, grande parte das fontes identificadas pela ICA podem ser decompostas em um conjunto de fontes esparsas locais que não são necessariamente independentes entre si. Além disso, as fontes identificadas pela LSCA aproximam muito melhor o sinal temporal observado nas regiões representadas por seus componentes do que as fontes identificadas pela ICA. Finalmente, uma análise mais elaborada utilizando a LSCA permite estimar também relações dinâmicas entre os componentes previamente identificados. Assim, a LSCA permite identificar relações clássicas bem como 
relações causais entre componentes do estado de repouso. As principais implicações desse resultado são que diferentes premissas permitem decomposições aproximadamente equivalentes, entretanto, critérios menos restritivos tais como esparsidade e localização permitem construir modelos mais compactos e biologicamente mais plausíveis.

Palavras-chave: Decomposição Esparsa, Ressonância Magnética Funcional, Estado de Repouso, Separação Cega de Fontes. 


\section{Abstract}

Vieira, G. Local Sparse Component Analysis: an Application to Funcional Magnetic Resonance Imaging. 2015. 104 f. Tese (Doutorado) - Instituto de Matemática e Estatística, Universidade de São Paulo, São Paulo, 2015.

This thesis presents Local Sparse Component Analysis (LSCA), a new method for analyzing resting state functional magnetic resonance imaging (fMRI) datasets. LSCA, a extension of Sparse Component Analysis (SCA), takes into account data spatial information to reconstruct temporal sources representing connected regions of significant activity. This study contains simulation data and real data. The simulated data were prepared to evaluate the LSCA in different scenarios. In the first scenario, the LSCA is compared with Principal Component Analysis (PCA) for detecting local sources under Gaussian white noise. Then, LSCA is compared with the expectation maximization algorithm (EM) for detecting the dynamics of local sources. Real data were collected for comparative and illustrative purposes. FMRI images from eleven healthy volunteers were acquired using a 3T MRI scanner during a resting state protocol. Images were preprocessed and analyzed using LSCA and Independent Components Analysis (ICA). LSCA components were compared with commonly reported ICA components. In addition, LSCA was applied for characterizing the dynamics of resting state networks. Simulated results have shown that LSCA is suitable for identifying local sparse sources. For real resting state FMRI data, LSCA is able to identify the same sources that are identified using ICA, allowing detailed functional connectivity analysis of the identified regions within and between components. This suggests that ICA resting state networks can be further decomposed into local sparse components that are not necessarily independent from each other. Moreover, LSCA sources better represent local FMRI signal oscillations than ISCA sources. Finally, brain connectivity analysis shows that LSCA can identify both instantaneous and causal relationships between resting state components. The main implication of this study is that independence and sparsity are equivalent assumptions in resting state FMRI. However, less restrictive criteria such as sparsity and source localization allow building much more compact and biologically plausible brain connectivity models.

Keywords: Sparse Decomposition, Functional Magnetic Resonance Imaging, Resting State, Blind Source Separation. 


\section{Sumário}

$\begin{array}{ll}\text { Lista de Abreviaturas } & \text { ix }\end{array}$

Lista de Símbolos $\quad$ xi

Lista de Figuras $\quad$ xiii

$\begin{array}{lll}\text { Lista de Tabelas } & \text { Xv }\end{array}$

1 Introdução $\quad 1$

1.1 Considerações Preliminares . . . . . . . . . . . . . . . . . . . . . 1

1.2 Revisão de Literatura . . . . . . . . . . . . . . . . . . . . . . 4

1.2.1 Conceitos Básicos de FMRI . . . . . . . . . . . . . . . . . . . 4

1.2 .2 O Estado de Repouso . . . . . . . . . . . . . . . . . . . . 5

1.2.3 Separação Cega de Fontes . . . . . . . . . . . . . . . . . . 9

1.2.4 Modelos Dinâmicos Espaço-Temporais . . . . . . . . . . . . . . 10

1.3 Formulação do Problema . . . . . . . . . . . . . . . . . . . . . . . . . 14

1.4 Contribuições . . . . . . . . . . . . . . . . . . . . . 15

1.4.1 Um Modelo para Separação Cega de Fontes Esparsas Locais . . . . . 15

1.4 .2 Uma Alternativa ao ICA . . . . . . . . . . . . . . . . . . . . . 16

1.4.3 Um Modelo Dinâmico para Fontes Esparsas Locais . . . . . . . . . . 17

1.5 Organização do Trabalho . . . . . . . . . . . . . . . . . . . . . . . . 19

2 Material e Métodos $\quad 21$

2.1 Análise de Componentes Esparsos Locais . . . . . . . . . . . . . . . . . . . . 21

2.1.1 Transformada Esparsa por WAVELETs . . . . . . . . . . . . . . . 21

2.1.2 Agrupamento com Restrição de Contiguidade . . . . . . . . . . . . . 26

2.1.3 Redução de Dimensionalidade . . . . . . . . . . . . . . . . . . . . . . 28

2.2 Dados Simulados . . . . . . . . . . . . . . . . . . . . . . . . . . 29

2.2.1 Modelo $1 \ldots \ldots \ldots \ldots \ldots$

2.2 .2 Modelo $2 \ldots \ldots \ldots \ldots$

2.3 Dados Reais . . . . . . . . . . . . . . . . . . . . . . . . . 31

2.3.1 Detalhes de Aquisição . . . . . . . . . . . . . . . . . . . 31

2.3.2 Processamento dos Dados . . . . . . . . . . . . . . . . . 32 
3 Resultados $\quad 35$

3.1 Simulações . . . . . . . . . . . . . . . . . . . . . . 35

3.1 .1 Modelo $1 \ldots \ldots \ldots \ldots$

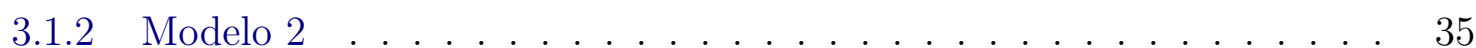

3.2 LSCA versus ICA . . . . . . . . . . . . . . . . . . . 36

3.2 .1 Resultados da ICA . . . . . . . . . . . . . . . . . . . . 37

3.2 .2 Resultados da LSCA . . . . . . . . . . . . . . . . . . 38

3.2 .3 Comparação entre LSCA e ICA . . . . . . . . . . . . . . . . . . 43

3.3 Análise de Componentes Dinâmicos Esparsos Locais . . . . . . . . . . . . . . 45

$\begin{array}{lll}4 & \text { Discussão } & 47\end{array}$

4.1 Simulações . . . . . . . . . . . . . . . . . . . . . . . . . . . . 47

$4.2 \quad \mathrm{LSCA}$ versus ICA . . . . . . . . . . . . . . . . . . 47

4.3 Análise de Componentes Dinâmicos Esparsos Locais . . . . . . . . . . . . . . 49

4.4 Considerações Finais . . . . . . . . . . . . . . . . . . . . . . . . 50

5 Conclusão $\quad 53$

5.1 Trabalhos Futuros . . . . . . . . . . . . . . . . . 54

$\begin{array}{ll}\text { A ICA e SCA } & 57\end{array}$

A.1 Separação Cega de Fontes . . . . . . . . . . . . . . . . . . . 57

A.1.1 Análise de Componentes Independentes . . . . . . . . . . . . . . 58

A.1.2 Análise de Componentes Esparsos . . . . . . . . . . . . . . . . . 60

$\begin{array}{ll}\text { B Wavelets } & 65\end{array}$

B.1 Tranformada Whyelets . . . . . . . . . . . . . . . . . 65

B.1.1 A Transformada WAVELET de Haar . . . . . . . . . . . . . . . . . . . 68

B.1.2 A Transformada WAVELET Multivariada . . . . . . . . . . . . . . 68

B.1.3 Pacotes Wavelets . . . . . . . . . . . . . . . . . 68

$\begin{array}{ll}\text { C O Algoritmo EM } & 71\end{array}$

$\begin{array}{ll}\text { Referências Bibliográficas } & 75\end{array}$ 


\section{Lista de Abreviaturas}

DMN Rede de Modo Padrão (Default Mode Network)

EM Maximização da Expectativa (Expectation Maximization)

FMRI Imageamento por Ressonância Magnética Funcional (Functional Magnetic Resonance Imaging)

FOV Campo de Visão (Field of View)

FWHM Largura Total à Meia Altura (Full Width at Half Maximum)

ICA Análise de Componentes Independentes (Independent Component Analysis)

LSCA Análise de Componentes Esparsos Locais (Local Sparse Component Analysis)

NMF Fatoração em Matrizes não Negativas (Non-Negative Matrix Factorization)

PCA Análise de Componentes Principais (Principal Component Analysis)

PDC Coerência Partial Direcionada (Partial Directed Coherence)

SCA Análise de Componentes Esparsos (Sparse Component Analysis)

TE Tempo de Eco (Time of Echo)

TR Tempo de Repetição (Time of Repetition) 


\title{
Lista de Símbolos
}

\author{
$\operatorname{cov}(x) \quad$ Covariância de $x$ \\ db Decibel \\ $\mathrm{E}(x) \quad$ Esperança de $x$ \\ $L^{2} \quad$ Espaço das funções $f(x)$ tal que $\int f d x<\infty$ \\ $\mathcal{N} \quad$ Distribuição Normal \\ $\langle$,$\rangle \quad Produto interno$ \\ $\mathbb{R} \quad$ Conjunto dos números reais \\ $\operatorname{vec}(x) \quad$ Empilhamento de $x$ \\ $\operatorname{var}(x)$ Variância de $x$ \\ vâr $(x) \quad$ Variância amostral de $x$ \\ $\mathbb{Z} \quad$ Conjunto dos números inteiros
}




\section{Lista de Figuras}

1.1 Regiões cerebrais identificadas utilizando a análise de componentes independentes em dados de FMRI durante o estado de repouso. As imagens representam porcentagem de variação de sinal e estão sobrepostas a um modelo de cérebro padrão. É interessante notar que embora o método ICA não visa separar componentes localmente, o padrão identificado representa regiões anatomicamente distintas e funcionalmente independentes. . . . . . . . . . . .

1.2 Regiões cerebrais identificadas utilizando a análise baseada em sementes. Nesse exemplo, a semente foi identificada pela área da mão localizada no córtex motor primário direito (desenho atípico). Observa-se que o mapa representa, preponderantemente, regiões cerebrais responsáveis pelo processamento motor, incluindo o sulco central, área motora suplementar e os giros pré e pós central bilateralmente. . . . . . . . . . . . . . .

2.1 As principais etapas da LSCA são: (i) aplicação de uma transformação esparsa baseada na transformada WAVELET, produzindo uma descrição em termos de séries temporais de coeficientes WAVELET, (ii) agrupamento com restrição de contiguidade das séries temporais de coeficientes WAVELET utilizando uma medida de dissimilaridade que agrupa somente coeficientes vizinhos, (iii) redução de dimensionalidade dos grupos por projeção ortogonal, produzindo estimadores da matriz de observação e das fontes do modelo (1.1) . . . . . . .

2.2 (a) Representação gráfica das colunas da matriz de observação A do Modelo 2 (b) Variância amostral do Modelo 2 para $N=500$ e SNR $=-19$ db. . . . .

3.1 Comparação da eficiência das técnicas LSCA e PCA em reconstruir a informação temporal das fontes. As linhas representam a correlação média entre as fontes simuladas $x_{k, t}$ e as fontes estimadas $\hat{x}_{k, t}$ em 30 simulações. . . . . .

3.2 Comparação da eficiência da LSCA e EM em reconstruir a informação temporal das fontes. As linhas representam a correlação média entre as fontes simuladas $x_{k, t}$ e as fontes estimadas $\hat{x}_{k, t}$ em 100 simulações. Barras verticais representam o erro padrão. . . . . . . . . . . . . . . . . . 
3.3 Linhas pontilhadas representam o valor teórico de PDC de acordo com o Modelo 2. Linhas sólidas representam o valor estimado de PDC utilizando o algoritmo EM com inicialização via LSCA. Linhas tracejadas representam o valor estimado de PDC utilizando o algoritmo EM com inicialização aleatória. 38

3.4 Componentes obtidos pela ICA. Os componentes estão ordenados de acordo com a porcentagem relativa de variância explicada. . . . . . . . . . . . .

3.5 Fração de energia acumulada no domínio dos voxels (em verde) e no domínio WAVELETS (em vermelho) para o conjunto de dados de FMRI durante o estado de repouso. A linha vertical em azul indica a fração de energia representada por $10 \%$ dos coeficientes com maior energia no domínio dos voxels (40\%) e no domínio WAVELETS $(80 \%) . \ldots \ldots \ldots \ldots$

3.6 Componentes identificados pela LSCA (da segunda até a quinta coluna) cuja dinâmica está significativamente correlacionada com a dinâmica de pelo menos um dos seis componentes identificados pela ICA com maior variabilidade (primeira coluna) . . . . . . . . . . . . . . . . . . .

3.7 Correlação de Pearson entre as fontes identificadas pela LSCA e ICA (corrigido para múltiplas comparações via FDR, $q<0.05)$. A menos das fontes que correspondem a componentes ruidosos devido a grande vasos (IC14 e IC28), todas as fontes identificadas pela ICA se correlacionam com pelo menos uma fonte identificada pela LSCA . . . . . . . . . . . . . . . . . . . . .

3.8 Correlação entre cada fonte estimada e sua respectiva série temporal para as análises pela ICA (A) e LSCA (B). A correlação média ao longo dos participantes é maior entre as fontes identificadas pela LSCA do que entre as fontes identificadas pela ICA (LSCA: $r=0.78$; ICA: $r=0.67 ; p<0.001$ ), sugerindo que a LSCA representa melhor a variabilidade dos dados que ICA. (C) Esta relação é ainda maior entre as fontes que representam as redes clássicas do estado de repouso. As correlações médias entre as fontes e suas respectivas séries temporais extraídas dos componentes da Figura 3.6 estão descritas em branco para a análise pela ICA e em cinza para a LSCA. (D) Valor de correlação máximo (eixo das $y$ ) entre cada fonte detectada pela ICA (eixo $x$ ) e todas fontes detectadas pela LSCA. . . . . . . . . . . . . . . . .

3.9 Análise da dinâmica dos componentes identificados através da LSCA. (a) Representação gráfica do fluxo de informação entre os componentes de acordo com a análise PDC das fontes. (b) Gráficos do valor de PDC entre as regiões ligadas por setas na Figura (a). Linhas tracejadas denotam o intervalo com 95\% de confiança para o valor médio de PDC (linhas sólidas). . . . . . . . .

A.1 Fontes simuladas de acordo com a equação (A.11) (a). Sinais observados de acordo com o modelo (A.14) (b). Gráfico de dispersão dos sinais observados de acordo com o modelo $($ A.14) $($ c) . . . . . . . . . . . . . . . . . . . 


\section{Lista de Tabelas}

3.1 Coordenadas MNI do máximo de atividade e nome anatômico dos componentes identificados pela LSCA . . . . . . . . . . . . . . . 40

A.1 Algoritmo fastICA para fontes independentes . . . . . . . . . . . . 60

A.2 Algoritmo SCA genérico para fontes esparsas . . . . . . . . . . . . 63 
xvi LISTA DE TABELAS 


\section{Capítulo 1}

\section{Introdução}

\subsection{Considerações Preliminares}

A ressonância magnética funcional (FMRI) é um método não invasivo de neuroimagem que permite observar simultaneamente a resposta hemodinâmica ao longo de todo o córtex cerebral [Ban12, HSM04]. Seu advento permitiu não somente analisar a atividade cerebral durante tarefas cognitivas complexas, como também desenvolver novas abordagens para estudar o chamado estado de repouso $\left[\mathrm{BMZ}^{+} 10\right]$. Enquanto a análise da atividade estuda a relação entre atividade cerebral e a presença ou ausência de estímulos, o estado de repouso descreve relações funcionais entre eventos que ocorrem em regiões anatomicamente remotas, permitindo tratamentos mecanicistas e interpretáveis de sistemas neuronais [VDHP10].

A principal característica do estado de repouso é a presença de correlações espontâneas, não induzidas pelo experimentador, entre regiões que compõem sistemas neurofisiológicos bem estabelecidos como, por exemplo, o córtex motor [DRB ${ }^{+} 06$, GSMD09]. Além de fornecer um modelo fundamental de organização funcional, o estado de repouso possui um protocolo de aquisição relativamente simples, por não depender de manipulações experimentais, o que viabiliza o uso clínico. Um exemplo importante do uso prático do estado de repouso é o mapeamento pré-cirúrgico de funções cognitivas de pacientes com tumores ou doenças neurológicas [FG10, GFM+07, Gre08, LSS13, RM11, WHHL12]. Técnicas de neuroimagem durante o estado de repouso tem permitido substituir métodos invasivos comumente utilizados para localizar regiões especializadas nestes pacientes. 


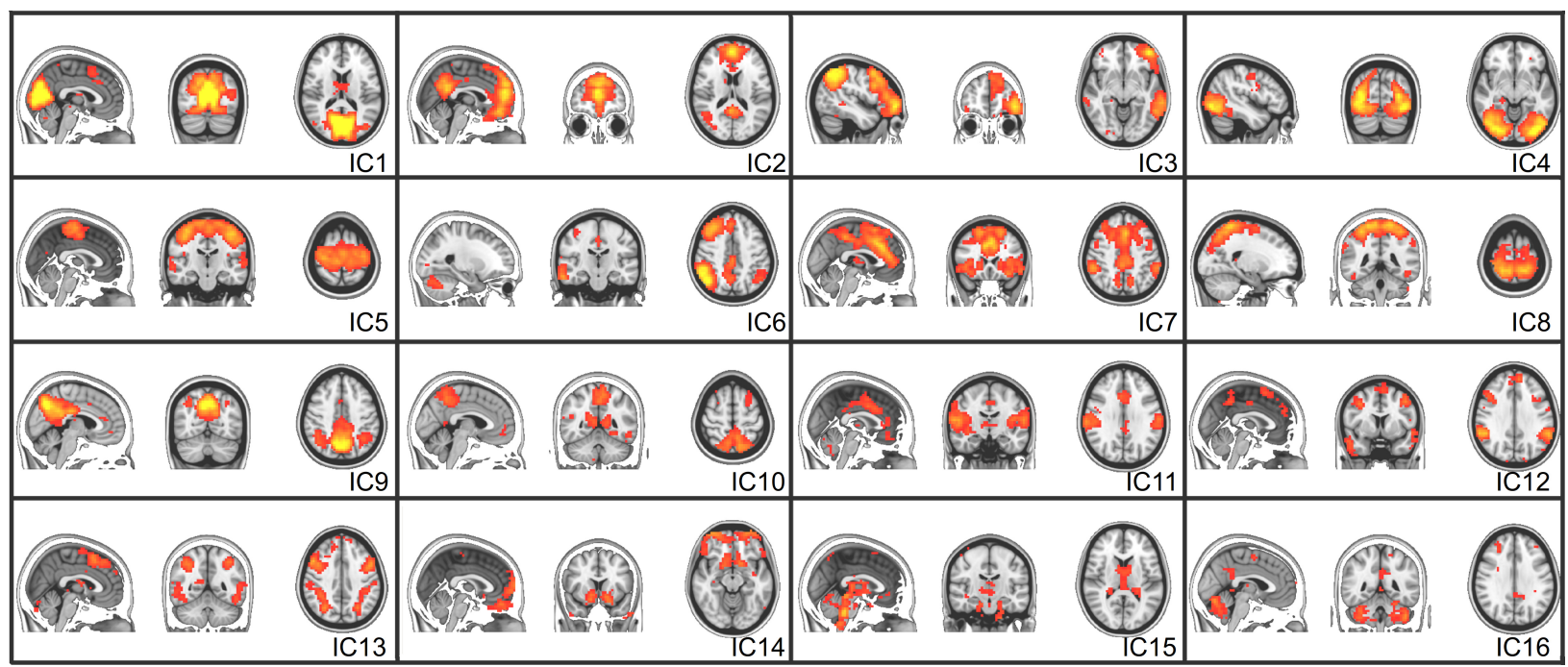

Figura 1.1: Regiões cerebrais identificadas utilizando a análise de componentes independentes em dados de FMRI durante o estado de repouso. As imagens representam porcentagem de variação de sinal e estão sobrepostas a um modelo de cérebro padrão. É interessante notar que embora o método ICA não visa separar componentes localmente, o padrão identificado representa regiões anatomicamente distintas e funcionalmente independentes.

Entretanto, inferir conectividade funcional ${ }^{1}$ no estado de repouso é uma tarefa complexa. Diferentemente da conectividade anatômica, que descreve conexões físicas em microescala, a conectividade funcional examina interações funcionais em macroescala, utilizando sinais neurofisiológicos de maneira não invasiva e, portanto, depende fortemente de técnicas de modelagem de dados [DG09, Hor03].

Em estudos de conectividade funcional, o principal desafio hoje é lidar com processos estocásticos compostos de subprocessos com localização espacial bem definida (Figura 1.1). Nesse sentido, os métodos convencionais de análise são ineficientes por não imporem restrições à extensão espacial de seus subprocessos. Há, portanto, necessidade de novos métodos para descrever conectividade funcional que apreciem melhor a informação espacial em imagens de FMRI.

Em geral, métodos de conectividade funcional no estado de repouso são divididos em dois tipos: métodos baseados em modelos e métodos exploratórios. Enquanto os métodos baseados em modelos se preocupam somente em estimar a conectividade utilizando séries temporais de regiões de interesse selecionadas a priori [ $\left.\mathrm{LGN}^{+} 09\right]$, os métodos exploratórios visam estimar, além da conectividade, a localização espacial das atividades cerebrais [RM11].

\footnotetext{
${ }^{1}$ termo empregado nesta tese para referir a relações funcionais causais ou instantâneas, estocásticas ou determinísticas.
} 
A abordagem baseada em modelos mais utilizada em estudos do estado de repouso é a análise de correlação baseada em semente. Nesta análise, um mapa de conectividade funcional voxel-à-voxel é obtido pelos valores do coeficiente de correlação entre a série temporal de uma região de interesse e as séries temporais do restante do cérebro [CSB10, DG09]. Embora seja fácil de implementar e interpretar, a análise baseada em sementes nem sempre caracteriza a variabilidade anatômica e funcional comumente observada em cérebros humanos. Potencialmente, o agrupamento de voxels sobre regiões de interesse implica em descartar informações essenciais para inferir corretamente a conectividade. Um outro problema é que a análise baseada em sementes omite regiões que permitiriam descrever corretamente a conectividade funcional de uma maneira geral. Assim, existe enorme interesse em abordagens que minimizam possíveis erros de seleção e/ou omissão de variáveis.

Abordagens exploratórias são preferidas quando é problemático escolher regiões de interesse de forma consistente $\left[\mathrm{MBL}^{+} 10\right]$. Atualmente, a abordagem exploratória mais utilizada em estudos do estado de repouso é a Análise de Componentes Independentes (ICA) cuja principal vantagem é decompor as imagens, não somente em componentes associados à atividade neuronal, mas também em componentes associados à artefatos [BU99, BDDS05]. Entretanto, a hipótese de que os componentes são independentes uns dos outros não é uma hipótese razoável do ponto de vista biológico. Além disso, por não possuir localização espacial bem definida, muitos componentes obtidos pela ICA são de difícil interpretação. Outros métodos exploratórios incluem a Análise de Componentes Principais (PCA) [WCLE05], Análise de Componentes Esparsos (SCA) [GTCB07], Fatoração em Matrizes não Negativas (NMF) [LHWY11], teoria dos grafos [FZB10, MBHZSZ12, WZH10], reconhecimento de padrão multivariado [CHHM09] e análise de agrupamento $\left[\mathrm{MYP}^{+} 09\right]$.

Entretanto, todos os métodos citados acima não tiram vantagem de uma propriedade intrínseca de sistemas neuronais e, consequentemente, imagens de FMRI: especialização funcional [BJBR09, GSS ${ }^{+}$06, $\mathrm{PWP}^{+}$99, GSS ${ }^{+}$06]. Imagens FMRI possuem uma enorme redundância espacial, de forma que voxels vizinhos são fortemente correlacionados. Várias abordagens exploram a redundância espacial em FMRI na tentativa de aumentar a relação sinal ruído das imagens. Nesse sentido, diversos autores utilizam filtros que atenuam variações de alta frequência ao logo do domínio espacial das imagens [FMP ${ }^{+}$22, HSM04, MR99, Smi04]. Po- 
rém, em estudos de conectividade funcional, filtros espaciais não resolvem os problemas de localização e seleção.

A fim de tratar adequadamente os problemas de localização, seleção e redundância espacial em estudos de conectividade funcional no estado de repouso, esta tese desenvolve um método denominado Análise de Componentes Esparsos Locais (LSCA) que leva em consideração a informação espacial para reconstruir a informação temporal de componentes espacialmente bem localizados. O princípio do LSCA é construir uma representação de subprocessos dinâmicos através do agrupamento local dos coeficientes da transformada WAVELET das imagens. O agrupamento é feito de maneira que os componentes identificados mantém a contiguidade espacial. Isto permite reduzir a dimensionalidade das imagens ao mesmo tempo que aumenta a relação sinal ruído. Resultados obtidos por simulação mostram que LSCA é superior aos métodos tradicionais na detecção de fontes espacialmente locais. Além disso, LSCA também é capaz de detectar regiões pertencentes às redes do estado de repouso, permitindo uma caracterização compacta e biologicamente plausível da dinâmica cerebral.

Apesar da motivação e dos principais resultados apresentados nesta tese se concentrarem no tema estado de repouso cerebral, o material desenvolvido aqui pode ser estendido sem muito esforço para outras aplicações que envolvam sistemas dinâmicos espaço-temporais.

\subsection{Revisão de Literatura}

\subsubsection{Conceitos Básicos de FMRI}

Atualmente, FMRI é a técnica de monitoramento neuronal mais utilizada em neurociência [HR02, MHB06]. Embora seu sucesso tenha sido determinado principalmente pela farta disponibilidade de equipamentos de ressonância magnética em diversas instituições de pesquisa, a FMRI se tornou indispensável no entendimento das propriedades hemodinâmicas cerebrais [Ban12, Log08]. Uma imagem de FMRI consiste de séries temporais de imagens tridimensionais coletas em intervalos fixos de tempo que variam entre 300 e 5000 milissegundos e com uma resolução espacial que varia entre $[0.5-7] \times[0.5-7] \times[0.5-7]$ milímetros cúbicos. Uma das principais causas da variabilidade do sinal de FMRI quando a técnica é utilizada 
próximo aos capilares é a variação da taxa de concentração de hemoglobina oxigenada em relação a concentração de hemoglobina desoxigenada [HSM04]. Alterações hemodinâmicas dessa natureza acontecem devido ao acoplamento neurovascular, ou seja, ao aumento do fluxo e volume sanguíneo em função da atividade neuronal [LW04].

Os primeiros estudos que utilizaram FMRI para processos neurais se concentraram, principalmente, em descobrir regiões onde os sinais de FMRI estariam associados a determinados tipos de estímulos. Utilizando um paradigma de estimulação visual, Kwong et al. [KBC $\left.{ }^{+} 92\right]$ observaram um aumento significativo da intensidade de sinal no córtex visual primário (V1) durante foto estimulação. Nesse mesmo estudo, alterações semelhantes no córtex motor primário (M1) foram observadas em uma tarefa de abertura e fechamento da mão. Embora o experimento de Kowng et al. $\left[\mathrm{KBC}^{+} 92\right]$ tenha sido realizado há mais de 20 anos, até hoje, a maioria dos experimentos de FMRI busca identificar quais regiões se ativam devido a presença ou ausência de um estímulo utilizando regressão linear simples [FAH ${ }^{+}$03, HSM04]. Esse princípio tem se demonstrado bastante importate e propiciado muitas descobertas sobre o funcionamento cerebral. Entretanto, ele é limitado, pois não se aplica a uma ampla variedade de protocolos experimentais, por exemplo, ao estado de repouso. Isso acontece porque a regressão linear simples ignora aspectos de conectividade que podem influenciar tanto respostas locais quanto padrões de atividade diretamente associados as condições experimentais e populações de estudo.

\subsubsection{O Estado de Repouso}

O estado de repouso em FMRI é um método que permite avaliar interações entre regiões cerebrais quando o indivíduo não está realizando uma tarefa específica [BYHH95, $\mathrm{CHA}^{+}$00, LMS98]. Durante o estado de repouso, os indivíduos são instruídos a relaxar e não pensar em nada específico enquanto as imagens de FMRI são coletadas [VDHP10]. Mesmo em repouso, o cérebro ainda está em atividade [FR07]. Estima-se que o cérebro humano em atividade cognitiva consuma somente cerca de $5 \%$ a mais de energia em relação ao repouso [RMS $\left.{ }^{+} 01\right]$. Portanto, muitas regiões apresentam ativações espontâneas que podem ser medidas por FMRI. O que é contra-intuitivo é o fato de que a atividade do estado de repouso contém muita informação a respeito da organização funcional do cérebro [FSV $\left.{ }^{+} 05\right]$. Por exemplo, 
regiões que coativam durante uma tarefa possuem sinais de FMRI correlacionados durante o repouso.

Estudos do estado de repouso supõem que as flutuações espontâneas de baixa frequência (entre 0,01 e 0,1 Hz) no sinal de FMRI reflitam a atividade cerebral [CHA $\left.{ }^{+} 01\right]$. No entanto, a verdadeira origem de tais oscilações não é completamente compreendida e muitos estudos indicam razões fisiológicas distintas para explicá-las, principalmente devido a limitada taxa de amostragem do sinal de FMRI que não possibilita identificar corretamente a frequência de processos respiratórios e cardíacos que poderiam, em princípio, introduzir um viés nas estimativas [BMB08, CG09]. Por outro lado, muitos autores defendem a hipótese de uma origem neuronal para as flutuações espontâneas de baixa frequência durante o estado de repouso [ $\left.\mathrm{VPF}^{+} 07\right]$. A principal evidência a favor dessa hipótese resulta da análise da conectividade de tais sinais, que permite identificar consistentemente sistemas neurofisiológicos bem conhecidos, sugerindo integração de regiões que supostamente possuem uma relação funcional estabelecida [BDDS05, DRB ${ }^{+} 06$, DG09, DLBDS ${ }^{+} 06$, GSMD09]. Outra evidência é o fato de que oscilações de baixa frequência em sinais de FMRI estão fortemente associadas às ocilações de sinais eletrofisiógicos [SL08]. Finalmente, é possível mostrar que ruídos fisiológicos como sinais cardíacos e respiratórios tem pouco impacto nas correlações de baixa frequência no estado de repouso $\left[\mathrm{CHA}^{+} 01\right]$.

A característica mais importante do estado de repouso é um padrão bem robusto de atividade correlacionada refletindo a organização funcional de vários sistemas cognitivos tais como motor, auditivo, visual, linguagem e memória [VDHP10]. A ideia de que o cérebro é uma rede integrada de regiões é vista por muitos pesquisadores como a chave para entender mecanismos cerebrais complexos de processamento de informação [GKRM03]. Além disso, por serem consistentemente estimadas em diversas condições, as redes do estado de repouso permitem examinar como associações entre regiões remotas se alteram em diferentes contextos neurofisiológicos, doenças neurológicas e distúrbios psiquiátricos, sem a necessidade de uso de tarefas cognitivas bem elaboradas [CHHM09, Gre08, LSS13]. Nesse sentido, vários estudos têm demonstrando que análises de conectividade no estado de repouso são mais sensíveis aos efeitos de doenças do que análises que procuram simplesmente observar diferenças regionais de ativação [CGS ${ }^{+} 11, \mathrm{HR} 11, \mathrm{HR} 13$, Row10, WN11]. 


\section{Conectividade no Estado de Repouso}

O cérebro é um conjunto de regiões que processam informações especializadas e se interconectam funcional e estruturalmente para integrá-las de maneira eficiente e organizada [BS09, RS10, SCKH04]. A conectividade funcional caracteriza a dependência temporal entre eventos neurofisiológicos em regiões remotas, sugerindo a existência de um canal de comunicação entre elas $\left[\mathrm{LGN}^{+}\right.$09]. A integração da informação de níveis mais básicos da rede neuronal para níveis mais complexos é a chave para entender processos cerebrais extremamente complicados que envolvem o processamento visual, motor, memória e motivação [TSE94]. Essa é a principal motivação para estudar a conectividade no nível de regiões, fornecendo modelos mecanicistas do padrão de organização do cérebro humano.

Os primeiros métodos de conectividade no estado de repouso fizeram uso da correlação devido a sua simplicidade de implementação e interpretação, buscando identificar oscilações espontâneas síncronas entre sinais de FMRI de diferentes regiões [BYHH95, LGN ${ }^{+}$09]. O método denominado análise baseada em semente consiste em selecionar uma região e calcular a correlação entre a série temporal desta região e as séries temporais das demais regiões da imagem de FMRI. Por exemplo, em Biswal et al. [BYHH95], a análise baseada em semente demonstrou uma forte sincronização entre regiões lateralmente homólogas do sistema motor utlizando uma semente localizada no córtex premotor, mais precisamente na área da mão, sugerindo uma forma de processamento local e integrado entre essas regiões. A ideia de conectividade entre áreas contralaterais foi explorada e replicada por diversos estudos não somente para regiões do sistema motor mas também para regiões de outros sistemas bem conhecidos em neurofisiologia [BV07]. A Figura 1.2 mostra os voxels cuja correlação entre a região semente (indicada pelo desenho atípico) é estatisticamente diferente de zero.

Obviamente, uma das limitações do método baseado em sementes é a necessidade de seleção de uma região semente a priori. Tradicionalmente, a semente é selecionada utilizando um experimento prévio capaz de delimitar a região de interesse. Entretanto, isso não exclui possíveis erros de seleção que resultam em modelos mal especificados, pois dificilmente um processo cognitivo é caracterizado por somente uma região. Uma outra limitação da análise baseada em sementes se deve ao fato de que a correlação caracteriza apenas relações bivariadas, excluindo modelos que expliquem as associações de maneira parcial. Por último, a 

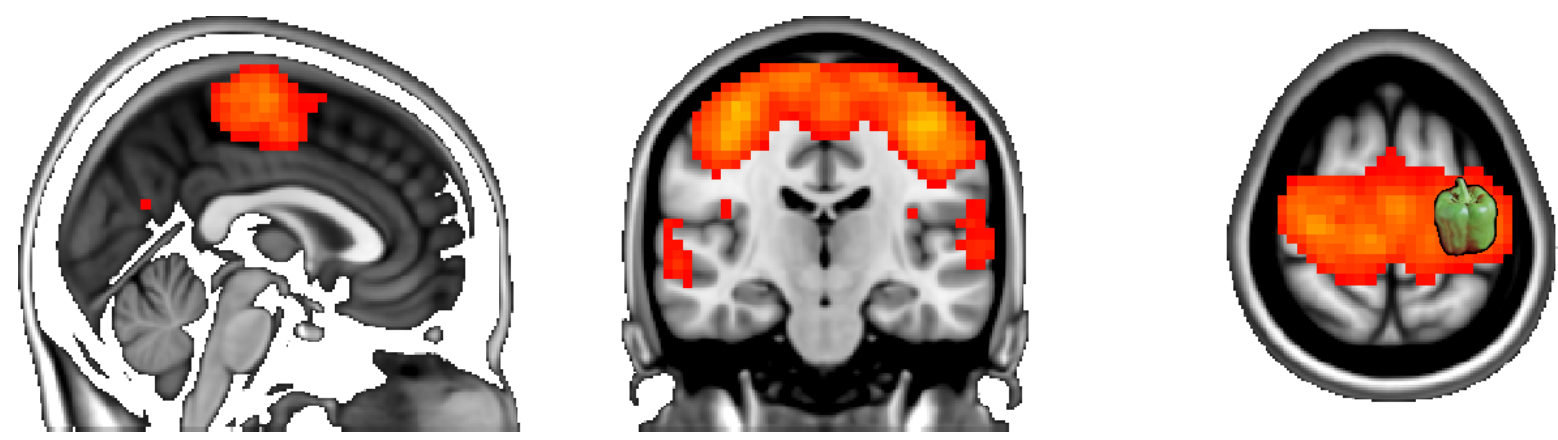

Figura 1.2: Regiões cerebrais identificadas utilizando a análise baseada em sementes. Nesse exemplo, a semente foi identificada pela área da mão localizada no córtex motor primário direito (desenho atípico). Observa-se que o mapa representa, preponderantemente, regiões cerebrais responsáveis pelo processamento motor, incluindo o sulco central, área motora suplementar e os giros pré e pós central bilateralmente.

análise de correlação é instantânea no sentido que ela não modela aspectos dinâmicos de conectividade e, portanto, não permite caracterizar o fluxo de informação entre os processos envolvidos durante o estado de repouso.

Abordagens exploratórias, por outro lado, permitem examinar a conectividade de uma maneira geral, sem a necessidade de delimitar previamente a localização das regiões envolvidas. Dentre os vários métodos que foram aplicados com esse propósito, a técnica de separação cega de fontes ICA é a mais popular, principalmente por ser bastante consistente [BDDS05, BU99, BMZ ${ }^{+}$10, $\mathrm{DRB}^{+}$06]. Entretanto, presumir independência entre os componentes é bastante restritivo, seja do ponto de vista estatístico ou biológico, pois dificulta comparar resultados entre estudos clínicos que frequentemente lidam com populações heterogêneas $\left[\mathrm{EFS}^{+}\right.$02]. Além disso, ICA não possui um método estável para estimar o número de componentes identificados [MS $\left.{ }^{+} 98\right]$. Na prática, a decisão sobre o número de componentes é bastante subjetiva e dependente da forma como o algoritmo foi implementado [CPA04].

Apesar das dificuldades, os resultados de ambos os métodos possuem bastante sobreposição. Dentre os resultados mais consistentes, encontram-se as redes do estado de repouso: regiões anatomicamente separadas, porém funcionalmente relacionadas. Até o momento, diversos autores identificaram uma série de redes e subredes utilizando diversos equipamentos de FMRI e formas de análise que convergem para uma rede motora, uma rede visual, uma rede atencional conectando os córtices frontal superior e parietal superior bilateralmente, uma rede de saliência contendo o córtex insular, parte do córtex temporal e o cíngulo anterior e a rede de modo padrão (DMN) que inclui os córtices: pré-cuneo, frontal medial, parietal inferior 
e temporal superior [BDDS05, BU99, $\mathrm{BMZ}^{+}$10, $\mathrm{DRB}^{+}$06, $\mathrm{DRB}^{+}$06, GKRM03, VDHP10].

É importante notar que as redes do estado de repouso representam sistemas cerebrais que foram descritos utilizando outras técnicas neurofisiológicas. Entretanto, muito mais atenção tem sido dada para as ativações espontâneas correlacionadas observadas entre as regiões da DMN [BAHS08, Fra05, GKRM03]. Um fato interessante envolvendo as regiões da DMN é que enquanto as regiões primárias cerebrais diminuem a atividade metabólica durante o estado de repouso, as regiões da DMN apresentam o oposto, sugerindo que elas são responsáveis pelo processamento basal do cérebro. Por isso, a atividade síncrona entre as regiões da DMN tem sido associada a processos complexos específicos da espécie humana como integração cognitiva e emocional da informação, e outros processos relacionados a consciência humana $\left[\mathrm{RMS}^{+}\right.$01]. Isso torna a DMN de especial interesse em estudos que buscam entender doenças neurológicas e psiquiátricas [GSRM04, Gre08].

Finalmente, outros estudos têm mostrado a existência de subredes dentro das redes do estado de repouso [vdHMLP08, vdHMK ${ }^{+} 09$ ]. Beckman et al. [BDDS05] mostraram associação entre sinais de regiões da rede visual com sinais de regiões do córtex estriado que são responsáveis pelo processamento da informação visual. Van den Heuvel e Hulshoff Pol [vdHPH10] mostraram que as regiões da rede motora podem ser organizadas de acordo com o padrão somatotópico das regiões do córtex motor. Xiong et al. [XMW $\left.{ }^{+} 09\right]$ mostram que o treinamento motor aumenta significantemente a conectividade entre regiões do córtex motor primário durante estado de repouso. Juntos, esses resultados sugerem que tais correlações representam, de fato, padrões de conectividade neuronal. Portanto, a conectividade entre tais regiões seria necessária para manter e pré-processar informações utilizadas pelas mesmas regiões durante uma tarefa específica.

\subsubsection{Separação Cega de Fontes}

Uma maneira conveniente de descrever toda variabilidade dos sinais de FMRI é adotar um modelo de separação cega de fontes no qual os sinais $\mathbf{z}(s, t)$ são amostrados discretamente $\mathbf{z}_{t}=\left[z_{1, t}, \ldots, z_{M, t}\right]^{\mathrm{T}}$ em $M$ localidades espaciais $s_{1}, \ldots, s_{M} \in D \subset \mathbb{R}^{d}$ em intervalos regulares de tempo. Supõe-se que $\mathbf{z}_{t}$ se deve, em última análise, a $K<M$ fontes fisiologicamente 
significativas, através de:

$$
\mathbf{z}_{t}=\mathbf{A} \mathbf{x}_{t}+\mathbf{v}_{t}
$$

em que $\mathbf{x}_{t}=\left[x_{1, t}, \ldots, x_{K, t}\right]^{\mathrm{T}}$ descrevem as fontes, $\mathbf{v}_{t}=\left[v_{1, t}, \ldots, v_{M, t}\right]^{\mathrm{T}}$ descrevem variações espaciais de pequena escala e $\mathbf{A}=\left[\mathbf{a}_{1} \cdots \mathbf{a}_{K}\right]$ é uma matriz de observação em que $\mathbf{a}_{i}=$ $\left[a_{i}\left(s_{1}\right), \ldots, a_{i}\left(s_{M}\right)\right]^{\mathrm{T}}$, para $1 \leq i \leq K$ e $a_{i}: \mathbb{R}^{d} \mapsto \mathbb{R}$, caracteriza como a informação de $\mathbf{x}_{t}$ se distribui ao longo do espaço.

O problema de separação cega de fontes consiste em reconstruir $\mathbf{X}=\left[\mathbf{x}_{1}, \ldots, \mathbf{x}_{N}\right]$ a partir de $\mathbf{Z}=\left[\mathbf{z}_{1}, \ldots, \mathbf{z}_{N}\right]$ considerando que $\mathbf{A}$ é, a princípio, desconhecido. Obviamente, a solução de problemas de separação cega de fontes é um problema mal posto a menos que haja conhecimento completo ou parcial das características geométricas ou estatísticas de $\mathbf{x}_{t}$ e/ou A. As duas premissas mais utilizadas são baseadas nos conceitos de independência e diversidade. Assumir que $\mathbf{x}_{t}$ consiste em um conjunto de fontes estatisticamente independentes e não gaussianas conduz à ICA. Sob essas premissas, as fontes estimadas pela ICA são iguais as fontes originais a menos de uma permutação de $\{1, \ldots, K\}$ e escala [Com94]. Entretanto, a tendência atual é explorar propriedades geométricas das distribuições das fontes. Por exemplo, é possível reconstruir as fontes assumindo que seu suporte seja limitado [PMJ95]. No geral, esse princípio permite reconstruir fontes esparsas baseado na ideia de que a diversidade dos dados permite identificar as fontes de maneira simples por meio de uma decomposição em componentes esparsos [ZP+ ${ }^{+}$1, GL06]. Nesse caso, o principal objetivo é obter uma transformação que preserve as relações lineares entre as fontes e, ao mesmo tempo, aumente a diversidade dos dados.

\subsubsection{Modelos Dinâmicos Espaço-Temporais}

Em estudos de conectividade em FMRI, há uma necessidade cada vez maior de se descrever com precisão como as regiões estão dinamicamente inter-relacionados. O principal desafio é lidar com modelos que empregam muitos parâmetros para descrever um grande número de variáveis que, no entanto, desfrutam de uma enorme redundância espacial. Além disso, em muitas aplicações, o foco se encontra na caracterização de interações sutis envolvendo um pequeno número de regiões em diferentes escalas espaciais. Uma maneira de tratar dados 
multivariados típicos em FMRI é fazer uma mudança de base buscando representar os dados com um número menor de componentes não nulos. Essa questão foi explorada no contexto de sistemas dinâmicos espaço-temporais por Wikle e Cressie [WC99] que propuseram um método de redução de dimensionalidade para modelar fenômenos espacialmente contínuos que evoluem no tempo. Em sua formulação original, o método usa uma base ortogonal, definida a priori, para expandir o kernel de redistribuição de uma equação de diferenças de tempo discreto e contínua no espaço em termos de uma combinação linear finita de componentes espaciais [CW02, WC99].

Considere a seguinte equação de diferenças de tempo discreto e contínua no espaço

$$
x_{t}(s)=\int_{D} h(s, u) x_{t-1}(u) d u+w_{t}(s)
$$

em que $x_{t}(s)$ e $w_{t}(s)$ são processos definidos em cada ponto $s$ do conjunto $D \subset \mathbb{R}^{d}$ e $t \in \mathbb{Z}_{>0}$. A função contínua $h: \mathbb{R}^{d} \times \mathbb{R}^{d} \rightarrow \mathbb{R}$ é denominada o kernel de redistribuição da equação (1.2) e define as interações entre os estados $x_{t}(s)$ e $x_{t-1}(u)$ para todo $s$ e $u \in D$.

Dado $\mathcal{B}=\left\{\phi_{k}\right\}_{k \in \mathbb{Z}}$, uma base completa e ortonormal de $L_{2}\left(\mathbb{R}^{d}\right)$, suponha que $x_{t}(s)$ seja aproximado por $K$ elementos (componentes espaciais) de $\mathcal{B}$

$$
x_{t}(s)=\sum_{k=1}^{K} x_{k, t} \phi_{k}(s) .
$$

Considere também a expansão dos demais elementos de (1.2) na base $\mathcal{B}$

$$
\begin{aligned}
h(s, u) & =\sum_{i=1}^{\infty} b_{i}(s) \phi_{i}(u), \\
w_{t}(u) & =\sum_{k=1}^{\infty} w_{k, t} \phi_{k}(u),
\end{aligned}
$$

em que $\left\{b_{i}(s): i=1,2, \ldots\right\}$ são funções paramétricas não estocásticas e $\left\{x_{j, t}: j=1,2, \ldots\right\}$ e $\left\{w_{k, t}: k=1,2, \ldots\right\}$ processos aleatórios de tempo discreto.

Substituindo (1.4) e (1.3) em (1.2) e integrando os dois lados da equação em $\phi_{i}(s) d s$ 
temos que

$$
\begin{aligned}
x_{i, t} & =\sum_{k=1}^{K}\left(\int_{D} \phi_{i}(s) b_{k}(s) d s\right) x_{k, t-1}+\sum_{k=1}^{\infty} w_{k, t} \int_{D} \phi_{i}(u) \phi_{k}(s) d s \\
& =\sum_{k=1}^{K} h_{i, k} x_{k, t-1}+w_{i, t},
\end{aligned}
$$

para $1 \leq i \leq K$, ou, na forma matricial,

$$
\mathbf{x}_{t}=\mathbf{H} \mathbf{x}_{t-1}+\mathbf{w}_{t}
$$

em que $\mathbf{x}_{t}=\left[x_{1, t}, \cdots, x_{K, t}\right]^{\mathrm{T}}$ representa uma série temporal multivariada, $\mathbf{w}_{t}=\left[w_{1, t}, \cdots, w_{K, t}\right]^{\mathrm{T}}$ representa um processo de inovações multivariado.

Um modelo mais geral supõe que as observações de $x_{t}(s)$ em $M$ localizações espaciais $s_{1}, \ldots, s_{M}$ são corrompidas por um ruído branco aditivo $\mathbf{v}_{t}$

$$
\begin{aligned}
z_{i, t} & =x_{t}\left(s_{i}\right)+v_{i} \\
& =\sum_{k=1}^{K} x_{k, t} \phi_{k}\left(s_{i}\right)+v_{i},
\end{aligned}
$$

para $1 \leq i \leq M$, ou, na forma matricial

$$
\mathbf{z}_{t}=\boldsymbol{\Phi}_{K} \mathbf{x}_{t}+\mathbf{v}_{t}
$$

em que $\boldsymbol{\Phi}_{K}=\left[\boldsymbol{\phi}^{1} \cdots \boldsymbol{\phi}^{K}\right]$ e $\boldsymbol{\phi}^{i}=\left[\phi_{i}\left(s_{1}\right), \cdots, \phi_{i}\left(s_{M}\right)\right]^{\mathrm{T}}$.

Generalizando as equações (1.6) e (1.8) para uma matriz de observação arbitrária, obtemse uma formulação em espaço de estados para observações espaciais $\mathbf{z}_{t}$ que dependem da evolução de um vetor latente $\mathbf{x}_{t}$ através de modelo linear gaussiano

$$
\begin{aligned}
& \mathbf{x}_{t}=\mathbf{H} \mathbf{x}_{t-1}+\mathbf{w}_{t} \\
& \mathbf{z}_{t}=\mathbf{A} \mathbf{x}_{t}+\mathbf{v}_{t}
\end{aligned}
$$

em que $\mathbf{z}_{t}$ é um vetor de dimensão $M$ de sinais observados, $\mathbf{x}_{t}$ é um vetor de dimensão $K$ de 
fontes não observadas, A é uma matriz de observação de dimensão $M \times K$ não conhecida, $\mathbf{H}$ é uma matriz de transição desconhecida de dimensão $K \times K$ que descreve a dinâmica das fontes, $\mathbf{w}_{t}$ é um processo de inovações e $\mathbf{v}_{t}$ é ruído aditivo. Tanto $\mathbf{w}_{t}$ quanto $\mathbf{v}_{t}$ são vetores aleatórios com distribuição gaussiana de média nula e matriz de covariância $\mathbf{Q}$ e $\mathbf{R}$, respectivamente. As matrizes $\mathbf{H}, \mathbf{A}, \mathbf{Q}$ e $\mathbf{R}$ e os vetores $\mathbf{x}_{t}$ precisam ser estimados utilizando $\mathbf{z}_{t}$ somente.

A redução de dimensionalidade por transformações ortogonais tem recebido atenção no processamento de sinais observados em diferentes pontos do espaço e tempo, por exemplo, em mensurações geofísicas e meteorológicas, em processamento de sinais e imagens e em epidemiologia [Cor09, FAD ${ }^{+}$11, MGRA98, TAGM03, WJBS04]. Embora as funções base $\left\{\phi_{k}\right\}_{k \in \mathbb{Z}}$ sejam conhecidas, a questão de como aproximar $x_{t}(s)$ em (1.3) permanece um grande desafio. Quando o número de observações é bem menor que $M$, os estados do sistema ou o kernel de redistribuição devem ser descritos em termos de um número reduzido de parâmetros. Por exemplo, [WC99] propõe o uso de funções de base em associação com WAVELETS em escalas espaciais menores para reduzir a complexidade de processos atmosféricos e oceânicos. Esta ideia foi apoiada em [DK07] e ampliada em [SDK09] que consideraram kernels de redistribuição mais genéricos, porém, homogêneos no espaço e no tempo.

Em estudos cuja formulação em espaço de estados é adotada, o algoritmo de maximização da expectativa (EM) tem sido a ferramenta mais utilizada para resolver $(1.9,1.10)$ para $\mathbf{x}_{t}$, pois sua solução converge pelo menos para um máximo local da sua função de verossimilhança [DLR77, SS82]. Tradicionalmente, o algoritmo é inicializado com um solução gerada aleatoriamente e, então prossegue reiterando seus dois principais passos até atingir o máximo da função de verossimilhança. O primeiro passo, o passo E, obtém uma solução para $\mathbf{x}_{t}$ calculando-se a esperança condicional de sua função de log-verossimilhança dado os dados e alguma estimativa para os parâmetros do modelo. Sobre a hipótese de gaussianidade a esperança condicional de $\mathbf{x}_{t}$ é obtida pelo suavisador de Rauch-Tung-Striebel [RST65]. No segundo passo, o passo M, os parâmetros do modelo são estimados pela maximização da função de log-verossimilhança condicional estimada no passo E. Entretanto, a performance do algoritmo EM decai rapidamente em função do número de variáveis do sistema modelado. Em muitos casos, a solução pode até se tornar indeterminada. Uma outra desvantagem do 
algoritmo EM é que a qualidade de seus estimadores dependem da inicialização adequada dos parâmetros do sistema, o que nem sempre é uma tarefa fácil de resolver.

No contexto de FMRI, Valdés-Sosa [VS04] estudou sistemas dinâmicos espaço-temporais no âmbito da análise funcional de dados, forçando o kernel de redistribuição variar suavemente ao longo do espaço. Esta exigência é obtida penalizando a rugosidade espacial do kernel de redistribuição utilizando uma versão discreta do operador Laplaciano no espaço. Porém, mesmo utilizando uma decomposição em valores singulares para reduzir a dimensionalidade dos dados, tal procedimento concentrou-se no exame da influência exercida na rede por um voxel somente, tornando o método bastante exploratório. Outra desvantagem é que, se por um lado a hipótese de suavidade permite estimar os parâmetros do modelo pela redução drástica da dimensionalidade espacial do modelo, por outro ela também reduz a resolução do kernel de redistribuição. Portanto, suavisar o kernel de redistribuição representa diluir a informação espacial das imagens e consequentemente reduzir a sensibilidade e especificidade do método na identificação de relações causais entre regiões que se localizam em escalas menores que a largura do operador Laplaciano.

\subsection{Formulação do Problema}

Muitas teorias em neurociência estão calcadas na segmentação do cérebro em centros funcionais especializados [Fri05, PF13]. Para alcançar soluções biologicamente mais plausíveis, esta tese considera duas características importantes em neurociência sobre o que se constitui importantes componentes cerebrais: (a) fontes que fornecem uma representação compacta (i.e. de dimensão relativamente reduzida) da dinâmica do cérebro durante o estado de repouso como um todo e (b) componentes que são espacialmente bem localizados, ou seja, cuja extensão aproxime a variação local do sinal de FMRI. De acordo com o modelo (1.1), essa ideia se traduz em $a_{i}$ 's bem localizadas, i.e. funções de espalhamento de baixa dispersão e suporte $\operatorname{supp}\left\{a_{i}\right\}=\left\{s \in \mathbb{R}^{d}: a_{i}(s) \neq 0\right\}$ conectado. Um exemplo clássico de função de espalhamento é dado pela função densidade de probabilidade Gaussiana. Nesse caso, quanto menor o desvio padrão da função, melhor é sua localização no espaço. Funções de espalhamento caracterizam a dependência espacial de voxels vizinhos devido ao alto grau de 
suavização da resposta hemodinâmica [DH12]. A modelagem por funções de espalhamento permitem caracterizar melhor a esparsidade intrínseca esperada em imagens de FMRI. Na prática LSCA combina conceitos de transformada WAVELETS [MH92], esparsidade de grupo [CP07] e um algoritmo de clusterização com restrição de contiguidade [Mur85] para alcançar uma representação esparsa que reduz eficientemente a dimensionalidade das imagens enquanto preserva suas características fisiológicas.

A aplicabilidade do algoritmo LSCA pode ser estendida para modelos dinâmicos espaçotemporais utilizando a formulação $(1.9,1.9)$ cuja solução clássica pode ser obtida por um algoritmo EM [DLR77]. Entretanto, nota-se que o algoritmo EM garante convergência somente para um máximo local e, portanto, a validade de seus estimadores depende fortemente de uma inicialização adequada dos estados e parâmetros do modelo [Wu83]. A ideia aqui é utilizar o algoritmo LSCA para inicializar os parâmetros do algoritmo EM, garantindo uma solução compacta em componentes dinâmicos espacialmente locais.

\subsection{Contribuições}

Esta tese consiste em três partes principais que resultam dos três artigos associados que foram publicados como parte do doutorado do autor [VAB14, VAB15, VASB15]. As próximas seções deste capítulo detalham cada um desses trabalhos.

\subsubsection{Um Modelo para Separação Cega de Fontes Esparsas Locais}

A solução de problemas de separação cega de fontes está intimamente ligada ao conhecimento parcial das características de $\mathbf{x}_{t}$ e/ou A. Técnicas de separação cega de fontes têm sido muito utilizadas em estudos de FMRI para estimar consistentemente conectividade instantânea entre regiões sem a necessidade de delinear regiões de interesse a priori [BU99, $\mathrm{BMZ}^{+}$10, $\left.\mathrm{BDDS} 05, \mathrm{CAC07}, \mathrm{DRB}^{+} 06, \mathrm{GTCB} 07\right]$. Métodos como PCA, ICA, SCA e NMF são capazes de decompor a imagem de FMRI em um conjunto de séries temporais que representam a dinâmica de seus respectivos componentes espaciais [WCLE05, BU99, GTCB07, LHWY11]. Embora a hipótese de independência não tenha relação direta com a de esparsidade, um fato interessante é que resultados obtidos por ICA 
e SCA são muito semelhantes [GTCB07]. Ambas as técnicas exibem um padrão de regiões anatomicamente segregadas, porém funcionalmente relacionadas.

Técnicas convencionais são inadequadas para caracterizar variações espacialmente locais, pois elas não impõem restrições na extensão espacial de seus componentes. A LSCA é uma alternativa que leva em conta tanto a informação anatômica quanto funcional por decompor imagens de FMRI em componentes esparsos com base em sua informação espacial localmente redundante [VAB14]. A LSCA está intimamente relacionado com a SCA [GL06, GTCB07, ZP+ $\left.{ }^{+} 01\right]$. Porém, enquanto a SCA assume esparsidade de uma maneira geral, a LSCA assume esparsidade num sentido mais restrito, impondo que cada componente espacial represente uma função de espalhamento que age somente sobre uma única fonte bem localizada. Isso significa que as fontes e os componentes espaciais podem ser facilmente identificados utilizando um algoritmo de agrupamento cuja medida de dissimilaridade limita espacialmente os elementos que serão agrupados [Mur85]. Além disso, a LSCA utiliza a transformada WAVELETS para aproveitar ao máximo o efeito suavisador da função de espalhamento e, consequentemente, aumentar a relação sinal ruído.

\subsubsection{Uma Alternativa ao ICA}

Os avanços recentes em técnicas para separação cega de fontes, particularmente na ICA e decomposições esparsas, tem aprimorado nossa capacidade de entendimento dos padrões de atividade cerebral durante o estado de repouso [GTCB07, VAB14, DRB ${ }^{+}$06]. Nesse contexto, a distinção entre fontes independentes e fontes esparsas é crucial. A primeira é a base para a ICA em que os dados são explicados como uma combinação linear de fontes estatisticamente independentes entre si. A segunda é a principal premissa por trás da SCA que é capaz de separar perfeitamente fontes que podem ser facilmente identificadas, mesmo que isso exija aplicar uma transformação esparsa nos sinais observados. Embora a ICA tenha se demonstrado eficiente em vários estudos nos últimos anos, o conceito de esparsidade é considerado mais versátil, pois ele não depende da estrutura estocástica das fontes [GL06, LCA03]. Em vez disso, ele depende somente de uma transformação com a capacidade de tornar os dados mais esparsos. Além disso, os componentes identificados pela ICA não possuem uma localização espacial bem definida e portanto não podem ser utilizados na construção de redes de 
conectividade funcional [Smi12].

Embora a distinção entre independência e esparsidade esteja bem definida teoricamente, não está claro se essa distinção possui algum valor prático na detecção de fontes em experimentos de FMRI durante o estado de repouso, visto que resultados similares têm sido reportados por diferentes autores utilizando uma ou outra técnica. Por exemplo, componentes contendo regiões da DMN e da rede atencional foram consistentemente detectadas utilizando tanto métodos baseados em independência quanto métodos baseados em esparsidade $\left[\mathrm{BMZ}^{+}\right.$10, VAB15, WZC13]. Além disso, recentemente, a efetividade da ICA em FMRI tem sido associada a sua habilidade de tratar fontes esparsas ao invés de fontes independentes $\left[\mathrm{DRT}^{+}\right.$09]. Essa hipótese resulta de simulações numéricas e resultados experimentais bem elaborados mostrando que a performance dos algoritmos mais utilizados em análises de imagens de FMRI - FastICA [Hyv99] e InfoMax [LCG10] - depende muito mais do grau de esparsidade das fontes que do grau de independência. Por outro lado, outro estudo mais recente contesta essa tese $\left[\mathrm{CPP}^{+} 13\right]$. Nesse artigo, os autores fazem uma revisão de [DRT $\left.{ }^{+} 09\right]$ e reportam uma série de argumentos mostrando que FastICA e InfoMax fazem o que está proposto: maximizar a independência entre as fontes.

Nesta tese, a LSCA é utilizado para mostrar que a hipótese de esparsidade em estudos de FMRI no estado de repouso é válida. A evidência é dada pela comparação direta entre as fontes detectadas utilizando a ICA e as fontes detectadas utilizando a LSCA no mesmo conjunto de imagens [VAB14]. Mostra-se que a LSCA pode identificar as mesmas fontes que são identificadas pela ICA. Além disso, grande parte das fontes identificadas pela ICA podem ser decompostas em um conjunto de fontes localmente esparsas que não são necessariamente independentes entre si. Mais importante, fontes identificadas pela LSCA aproximam muito melhor o sinal temporal observado nas regiões representadas por seus componentes em relação as fontes identificadas pela ICA.

\subsubsection{Um Modelo Dinâmico para Fontes Esparsas Locais}

Uma limitação ainda mais importante das técnicas atuais de separação cega de fontes é estimar somente interações instantâneas entre os diferentes componentes. Embora esse fato seja bem conhecido, suas implicações são frequentemente ignoradas [Fri11, Smi12]. Devido 
a natureza do sinal de FMRI, interações dinâmicas não podem ser descritas separadamente no espaço ou no tempo, especialmente se o foco é caracterizar alterações espaciais associados a um pequeno número de regiões de interesse. Nesse contexto, estimar a origem espacial de fontes utilizando um número reduzido de amostras temporais é problemático especialmente em condições desfavoráveis de relação sinal-ruído.

Embora a mudança de base promovida por Wikle and Cressie [WC99] permita entender melhor sistemas de dimensão elevada, ela de forma alguma garante representações esparsas. Representações esparsas são a chave para descritores estatisticamente robustos, como tem sido demostrado na resolução de problemas de modelagem [MGB+ 09], deconvolução [TBM79] e reconstrução de imagens [Tin14], separação cega de fontes [ZP $\left.{ }^{+} 01\right]$, e algoritmos iterativos [PS82, Saa03]. Esses métodos obtêm representações esparsas pela minimização de uma função custo penalizada, assumindo um compromisso entre o ajuste do modelo e o número de elementos descritores do sinal observado [FGT12].

Recentemente, o conceito de esparsidade tem sido explorado de uma maneira mais restrita utilizando a ideia de penalização de grupo $\left[\mathrm{BJM}^{+} 12\right]$. Diferentemente da abordagem tradicional que penaliza as variáveis do modelo individualmente, na penalização de grupo, grupos de variáveis são penalizados simultaneamente pela minimização de uma função custo que inclui um termo quadrático representando o erro de aproximação e um termo de regularização considerando informações a priori suficientes para induzir esparsidade de grupo $\left[\mathrm{HZ}^{+} 10\right]$. Tais informações comumente refletem relações funcionais intrínsecas entre as variáveis do modelo. Por exemplo, em FMRI, uma série temporal representa um conjunto de observações sucessivas sobre o mesmo voxel, portanto, podem ser penalizadas conjuntamente.

A LSCA estende modelos dinâmicas espaço-temporais de dimensão reduzida utilizando uma formulação por espaço de estados que é apropriada para dados de FMRI [VAB15]. Utilizando a transformada espacial WAVELETS e o princípio de esparsidade de grupo, a LSCA é capaz de caracterizar a variabilidade temporal de fontes espacialmente bem localizadas utilizando um conjunto reduzido de séries temporais de coeficientes. A redução de dimensionalidade obtida pelo operador de limiarização vetorial permite que as fontes e os demais parâmetros do modelo sejam estimados consistentemente utilizando um algoritmo EM. 


\subsection{Organização do Trabalho}

O capítulo 2 apresenta os conceitos necessários para a compreensão do restante do texto. Nas primeiras sessões, as três etapas básicas de um algoritmo de SCA são descritas: a transformada esparsa, o agrupamento e a redução de dimensionalidade. O capítulo 2 também descreve os modelos que são utilizados para construir os dados simulados assim como os métodos de pré-processamento dos dados reais.

O capítulo 3 apresenta os resultados de vários estudos envolvendo a LSCA. Inicialmente, dois estudos mostram que a LSCA é melhor que técnicas clássicas de processamento de sinais. No primeiro estudo a LSCA é comparado com a PCA na caracterização de fontes gaussianas esparsas. No segundo estudo, simulações numéricas de modelos dinâmicos com fontes esparsas locais mostram que a inicialização do algoritmo EM utilizando a LSCA supera a abordagem clássica, baseada em inicialização aleatória, permitindo estimar completamente todas as variáveis do modelo $(1.9,1.10)$ mesmo sob condições desfavoráveis de relação sinal ruído. Ainda no capítulo 3, a LSCA é sistematicamente comparado com a ICA utilizando dados reais de FMRI durante o estado de repouso. Nesse estudo, compara-se a capacidade de localização e reconstrução das fontes da LSCA em relação à ICA. Finalmente, a LSCA é aplicada sobre o mesmo conjunto de imagens de FMRI durante o estado de repouso visando caracterizar sua dinâmica temporal.

O capítulo 4 discute os resultados apresentados no capítulo 3 e o capítulo 5 conclui as principais implicações do método apresentado nesta tese bem como suas principais limitações. No final, apresenta-se possibilidades de estudos futuros que envolvem representações esparsas de sistemas dinâmicos espaço-temporais com potenciais aplicações em FMRI.

O texto também contém três apêndices. No apêndice A são discutidas noções básicas de separação sega de fontes, com ênfase em ICA e SCA. No apêndice B, a transformada WAVELET, base das transformações esparsas utilizadas nesta tese, é apresentada. O apêndice C introduz o algoritmo EM da forma que é implementado nesta tese. 


\section{Capítulo 2}

\section{Material e Métodos}

\subsection{Análise de Componentes Esparsos Locais}

Nesta seção, o objetivo é desenvolver um algoritmo para estimar as fontes $\mathbf{x}_{t}$ e a matriz de observação A do modelo (1.1), sob a hipótese de que as colunas de A representam funções de espalhamento bem localizadas. Uma descrição sucinta do presente algoritmo pode ser observada na Figura 2.1.

\subsubsection{Transformada Esparsa por WAVELETS}

Dado $\left\{\phi_{m}\right\}_{1 \leq m \leq M}$ uma base do domínio WAVELETS em $\mathbf{R}^{M}$, o primeiro passo é calcular a transformada WAVELETS da matriz de observações $\mathbf{Z} \equiv\left(z_{m, t}\right)_{m, t}$ para $1 \leq m \leq M$ e $1 \leq t \leq T$

$$
\hat{\mathbf{Z}} \equiv\left(\hat{z}_{m, t}\right)_{m, t}=\left(\left\langle\mathbf{z}_{t}, \phi_{m}\right\rangle\right)_{m, t}=\mathbf{\Phi} \mathbf{Z}
$$

em que $\boldsymbol{\Phi}$ é uma matriz ortonormal $M \times M$, cujas linhas são definidas por $\phi_{m}$ 's. Utilizando a mesma notação tem-se que $\hat{\mathbf{Z}}=\hat{\mathbf{S}}+\hat{\mathbf{V}}$, em que $\mathbf{S}=\mathbf{A X}$, e $\mathbf{Z}=\mathbf{S}+\mathbf{V}$. A transformação $\boldsymbol{\Phi}$ deve ser escolhida tal que um agrupamento apropriado das linhas de $\hat{\mathbf{S}}$ forneça uma boa aproximação para as linhas de $\mathbf{X}$. Antes disto, porém, $\hat{\mathbf{S}}$ precisa ser estimado utilizando a hipótese de espasidade; Isto significa encontrar uma representação esparsa que capture os graus de liberdade intrínsecos de $\hat{\mathbf{Z}}$.

Considerando que $\mathbf{s}_{t}=\mathbf{A} \mathbf{x}_{t}$ admite uma representação esparsa pertencente a $\mathbf{B}_{1,1}^{s}$, um 


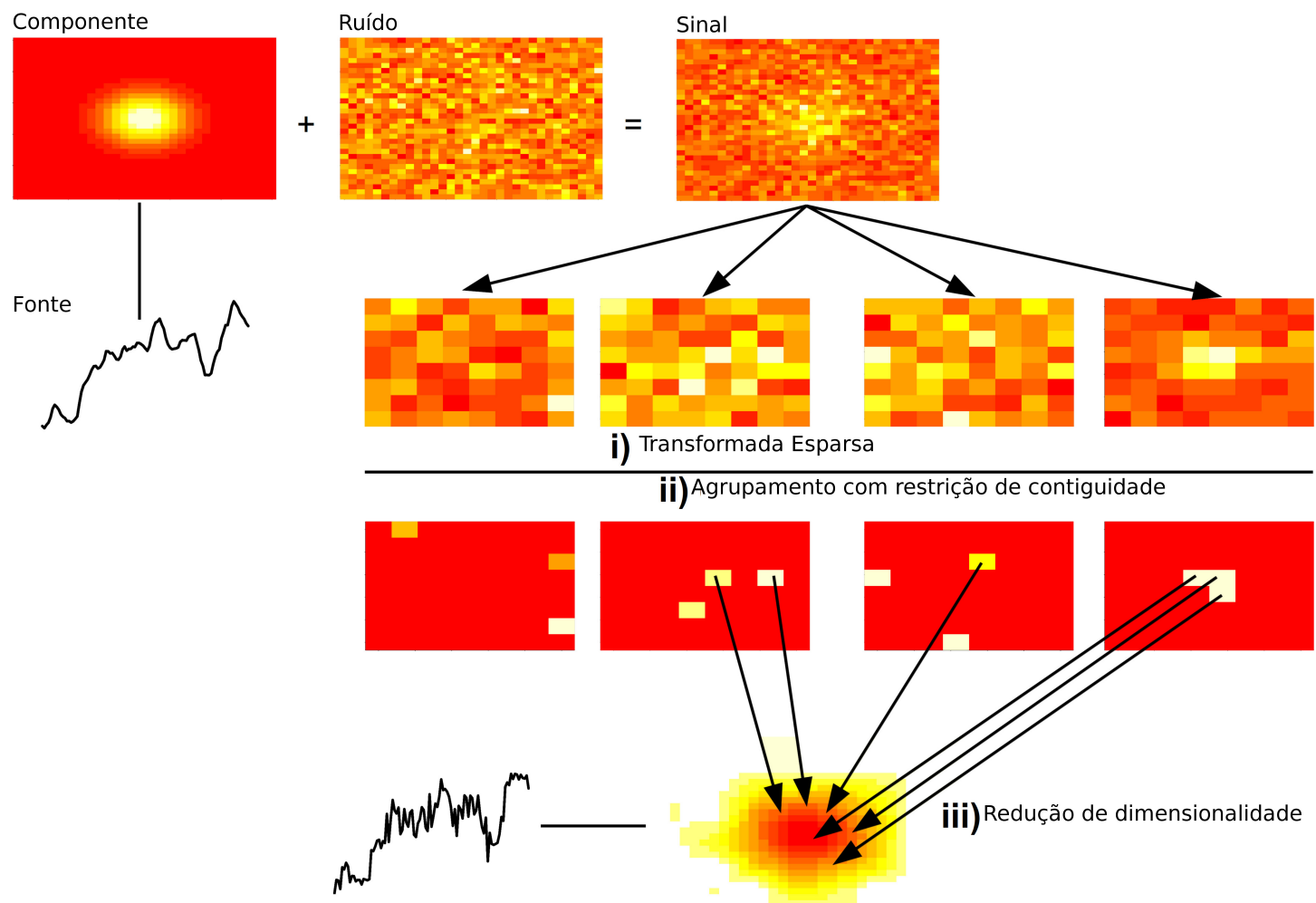

Figura 2.1: As principais etapas da LSCA são: (i) aplicação de uma transformação esparsa baseada na transformada WAVELET, produzindo uma descrição em termos de séries temporais de coeficientes WAVELET, (ii) agrupamento com restrição de contiguidade das séries temporais de coeficientes WAVELET utilizando uma medida de dissimilaridade que agrupa somente coeficientes vizinhos, (iii) redução de dimensionalidade dos grupos por projeção ortogonal, produzindo estimadores da matriz de observação e das fontes do modelo (1.1). 
caso particular de espaço de Besov [Mal08], a aproximação de $\mathbf{z}_{t}$ por $\mathbf{s}_{t} \in \mathbf{B}_{1,1}^{s}$ pode ser obtida minimizando o seguinte funcional

$$
f\left(\mathbf{s}_{t}\right)=\left\|\mathbf{z}_{t}-\mathbf{s}_{t}\right\|_{2}^{2}+\left\|\mathbf{s}_{t}\right\|_{s, 1}=\left\|\mathbf{z}_{t}-\mathbf{s}_{t}\right\|_{2}^{2}+\sum_{m} \lambda_{m}\left|\hat{s}_{m, t}\right|
$$

em que $\left\|\mathbf{s}_{t}\right\|_{s, 1}$ é a norma $\mathbf{B}_{1,1}^{s}$ do vetor $\mathbf{s}_{t}, \hat{s}_{m, t}=\left\langle\mathbf{s}_{t}, \phi_{m}\right\rangle$ e $\lambda_{m}>0$ para $1 \leq m \leq M$ são parâmetros regularizadores [DDDM03].

Para cada $t$, a equação acima representa um funcional coercivo e estritamente convexo. Isso significa que a equação (2.2) contém uma única solução e, portanto, um único mínimo global. Quando $\lambda_{m}=\lambda$, o vetor solução de (2.2) é obtido pela aplicação do operador de limiarização suave no conjunto de observações transformadas [DJKP95]

$$
\hat{s}_{m, t}=\operatorname{sign}\left(\hat{z}_{m, t}\right) \max \left(\left|\hat{z}_{m, t}\right|-\lambda, 0\right)
$$

Como $\hat{s}_{m, t_{0}}=0$ não implica $\hat{s}_{m, t_{1}}=0$ para $t_{0} \neq t_{1}$, o estimador dado pela equação $(2.3)$ não garante a esparsidade de $s_{t}$ ao longo do tempo mesmo para valores grandes de $\lambda$. Para superar esse problema, LSCA agrupa os coeficientes $\hat{s}_{m, t}$ ao longo do tempo e utiliza um regularizador de grupo para solucionar (2.2) no domínio WAVELETS

$$
\min _{\hat{\mathbf{s}}^{m}} \frac{1}{2}\left\|\hat{\mathbf{z}}^{m}-\hat{\mathbf{s}}^{m}\right\|_{2}^{2}+\lambda_{m}\left\|\hat{\mathbf{s}}^{m}\right\|_{2}
$$

em que $\hat{\mathbf{z}}^{m}$ e $\hat{\mathbf{s}}^{m}$ representam as $m$-ésimas linhas de $\hat{\mathbf{Z}}$ e $\hat{\mathbf{S}}$ respectivamente. Assim, para cada $\lambda_{m}$, a solução de (2.4) é dada pelo operador de limiarização suave vetorial [CW05, WNF09]

$$
\hat{\mathbf{s}}^{m}=\frac{\max \left(\left\|\hat{\mathbf{z}}^{m}\right\|_{2}-\lambda_{m}, 0\right)}{\left\|\hat{\mathbf{z}}^{m}\right\|_{2}} \hat{\mathbf{z}}^{m} .
$$

\section{Nível de Ruído Aditivo}

Um passo importante do algoritmo LSCA é a definição de um valor adequado para o parâmetro de regularização $\lambda_{m}$ que determina o limiar do operador vetorial de limiarização suave (2.5). Como regra básica, os limiares devem ser escolhidos um pouco acima do nível máximo de ruído. Assim, os coeficientes que representam ruído tem grande probabilidade 
de estar abaixo desse limiar. Esta sessão propõe um estimador para a variância do ruído supondo normalidade, independência e homocedasticidade.

Considere a decomposição de $\mathbf{z}_{t}=\mathbf{s}_{t}+\mathbf{v}_{t}$ no domínio WAVELETS

$$
\hat{\mathbf{z}}_{t}=\hat{\mathbf{s}}_{t}+\hat{\mathbf{v}}_{t}
$$

em que $\hat{\mathbf{v}}_{t}=\boldsymbol{\Phi} \mathbf{v}_{t}$. Para $\mathbf{v}_{t} \sim \mathcal{N}\left(\mathbf{0}, \sigma^{2} \mathbf{I}_{N \times N}\right)$, devido a ortogonalidade de $\boldsymbol{\Phi}$, tem-se que $\hat{\mathbf{v}}_{t} \sim \mathcal{N}\left(\mathbf{0}, \sigma^{2} \mathbf{I}_{N \times N}\right)$. Supondo que $\mathbf{z}_{t}$ admite uma representação esparsa em relação à transformação $\boldsymbol{\Phi}$, é esperado que a maior parte de $\left\{\hat{\mathbf{s}}^{m}\right\}_{\forall m}$ seja nula, ou seja, a maior parte de $\left\{\hat{\mathbf{z}}^{m}\right\}_{\forall m}$ seja ruído. Esta observação motiva o seguinte estimador de $\sigma^{2}$

$$
\hat{\sigma}^{2}=\operatorname{median}_{\forall m} \operatorname{vâr}\left\{\hat{z}_{m, t}\right\}
$$

em que vâr denota a variância amostral ao longo do tempo.

Não é difícil notar que $\hat{\sigma}^{2}$ é um estimador não viesado de $\sigma^{2}$ se pelo menos $50 \%$ das linhas de $\hat{\mathbf{S}}$ forem nulas, isto é, $\#\left\{m: \hat{\mathbf{s}}^{m}=\mathbf{0}\right\} / M>0.5$.

\section{Parâmetro de Regularização}

O estimador $\hat{\sigma}^{2}$ acima permite definir o parâmetro de regularização $\lambda$ através do seguinte resultado. No caso em que $\hat{s}_{m, t}=0$, tem-se

$$
\operatorname{var}\left\{\hat{z}_{m, t}\right\}=\sigma^{2}
$$

No caso em que $\hat{s}_{m, t} \neq 0$, tem-se

$$
\operatorname{var}\left\{\hat{z}_{m, t}\right\}=\operatorname{var}\left\{\hat{s}_{m, t}\right\}+\sigma^{2}
$$

Portanto, decidir se $\hat{z}_{m, t}$ em função de $t$ é ruído ou não equivale ao seguinte teste de hipóteses

$$
\begin{cases}\text { H0 }: & \operatorname{var}\left\{\hat{z}_{m, t}\right\}=\sigma^{2} \\ \text { H1 }: & \operatorname{var}\left\{\hat{z}_{m, t}\right\}>\sigma^{2}\end{cases}
$$


Para $\left\{\hat{z}_{m, t}\right\}_{\forall t}$ amostras independentes e normalmente distribuídos, tem-se, sob H0, que

$$
\frac{(N-1) \operatorname{vâr}\left\{\hat{z}_{m, t}\right\}}{\sigma^{2}} \sim \chi_{N-1}^{2}
$$

Isto permite construir o seguinte intervalo com $(1-\alpha) \%$ de confiança para $\sigma^{2}$

$$
\left[\frac{(N-1) \hat{\sigma}^{2}}{\chi_{1-\alpha / 2, N-1}^{2}}, \frac{(N-1) \hat{\sigma}^{2}}{\chi_{\alpha / 2, N-1}^{2}}\right],
$$

em que $\chi_{\gamma, N-1}^{2}$ é o $\gamma$-ésimo quantil da distribuição qui-quadrado com $N-1$ graus de liberdade.

Supondo que $E\left\{\hat{\mathbf{z}}_{t}\right\}=\mathbf{0}$, caso contrário, considere o processo estocástico definido por $\hat{\mathbf{z}}_{t}-E\left\{\hat{\mathbf{z}}_{t}\right\}$, tem-se que

$$
\left\|\hat{\mathbf{z}}^{m}\right\|_{2}=(N-1) \operatorname{vâr}\left\{\hat{z}_{m, t}\right\}
$$

As equações (2.11) e (2.12) motivam o seguinte estimador de $\lambda$

$$
\lambda=\frac{(N-1)^{2} \hat{\sigma}^{2}}{\chi_{\alpha / 2, N-1}^{2}}
$$

$\operatorname{com} \alpha=0.05 / M$.

\section{Extensão para Pacotes WAVelets}

Para facilitar a notação, suponha que $M=2^{J}$, para $J>0$, e $d=1$. Seja $\mathcal{L}$ uma biblioteca de pacotes WAVELETS. Fixado $t$, considere a seguinte análise de multirresolução espacial de $\mathbf{z}_{t}$

$$
\mathbf{z}_{t}=\sum_{(j, n) \in \mathbb{P}} \sum_{k=0}^{2^{j}-1} w_{j, n, k, t} \mathbf{w}_{j, n, k}
$$

em que $\mathbb{P}=\{(j, n)\} \subset \mathbb{N} \times \mathbb{N}$ representa uma coleção de índices e $\mathbf{w}_{j, n, k}=\left[W_{n}\left(2^{j} s_{1}-\right.\right.$ $\left.k), \ldots, W_{n}\left(2^{j} s_{M}-k\right)\right]^{\mathrm{T}}$, para $W_{n} \in \mathcal{L},(j, n) \in \mathbb{P}$ e $1 \leq k \leq 2^{J}$. Quando $\mathbb{P}$ é tal que os intervalos $\left[2^{j} n, 2^{j}(n+1)\right)$ formam uma cobertura disjunta de $\left[0,2^{J}\right)$, sabe-se que os vetores $\mathbf{w}_{j, n, k}$ formam uma base completa e ortonormal de $\mathbb{R}^{M}$ [CW92].

Dentre todas as bases geradas por $\mathcal{L}$, é possível obter uma base completa e ortonormal ótima no sentido de que a base priorize os componentes de $\mathbf{z}_{t}$ de maior variância. Para isso, é necessário definir um critério que julgue a importância de cada pacote WAVELET, 
$\mathbf{W}_{j, n}=\left\{\mathbf{w}_{j, n, 1}, \ldots, \mathbf{w}_{j, n, 2^{j}-1}\right\}$, em (2.14). Devido a ortonormalidade dos $\mathbf{w}_{j, n, k}$ 's, tem-se que a potência $\mathrm{E}\left(\mathbf{z}_{t}^{\mathrm{T}} \mathbf{z}_{t}\right)$ de $\mathbf{z}_{t}$ é a soma das potências dos coeficientes associados à sua transformada WAVELETS

$$
\begin{aligned}
\mathrm{E}\left(\mathbf{z}_{t}^{\mathrm{T}} \mathbf{z}_{t}\right) & =\mathrm{E}\left(\sum_{(j, n) \in \mathbb{P}} \sum_{k=0}^{2^{j}-1} w_{j, n, k, t} \mathbf{w}_{j, n, k}^{\mathrm{T}} \sum_{(j, n) \in \mathbb{P}} \sum_{k=0}^{2^{j}-1} w_{j, n, k, t} \mathbf{w}_{j, n, k}\right) \\
& =\mathrm{E}\left(\sum_{(j, n) \in \mathbb{P}} \sum_{k=0}^{2^{j}-1}\left(w_{j, n, k, t}\right)^{2} \mathbf{w}_{j, n, k}^{\mathrm{T}} \mathbf{w}_{j, n, k}\right) \\
& =\sum_{(j, n) \in \mathbb{P}} \sum_{k=0}^{2^{j}-1} \mathrm{E}\left(w_{j, n, k, t}^{2}\right)=\sum_{(j, n) \in \mathbb{P}} \sum_{k=0}^{2^{j}-1} \operatorname{var}\left(w_{j, n, k, t}\right),
\end{aligned}
$$

em que $\operatorname{var}(x)$ representa a variância de $x$. A última igualdade na equação (2.15) se deve ao fato de que $\mathrm{E}\left(\mathbf{z}_{t}\right)=\mathbf{0}$ implica $\mathrm{E}\left(w_{j, n, k, t}\right)=0$ para todo $(j, n) \in \mathbb{P}$ e $1 \leq j \leq 2^{J}$.

Como $\operatorname{var}\left(w_{t, j, n, k}\right)=\sigma^{2}$ se e somente se $w_{t, j, n, k}$ for ruído, definimos a seguinte função custo

$$
C_{\sigma^{2}}\left(\left\{\mathbf{W}_{j, n}\right\}\right)=\sum_{k=1}^{2^{j}-1} \mathbf{1}\left(\operatorname{vâr}\left(w_{j, n, k, t}\right) \geq \sigma^{2}\right),
$$

denominada função custo de limiarização dinâmica. Observa-se que $C_{\sigma^{2}}\left(\left\{\mathbf{W}_{j, n}\right\}\right)$ é aditiva e, portanto, pode ser utilizada por um algoritmo de seleção de base com complexidade linear em $M$ [Vid99a].

\subsubsection{Agrupamento com Restrição de Contiguidade}

O próximo passo consiste em identificar cada componente espacial $\mathbf{a}_{k} \mathbf{x}^{k}$, em que $\mathbf{a}_{k}$ é a $k$-ésima coluna de $\mathbf{A}$ e $\mathbf{x}^{k}$ é a $k$-ésima linha de $\mathbf{X}$, utilizando as séries temporais $\hat{\mathbf{s}}^{m}$ que sobreviveram o operador de limiarização vetorial em 2.5. Para isso, evoca-se a premissa de que os componentes são bem localizados no espaço e, portanto, os padrões geométricos das colunas da matriz de observação A podem ser perfeitamente descritos por coeficientes WAVELETS espacialmente conectados. Isso significa que os componentes espaciais do modelo (1.1) podem ser determinados por um algoritmo de agrupamento que força contiguidade espacial [Mur85]. Na LSCA, contiguidade espacial é obtida utilizando um algoritmo de agrupamento hierárquico com ligação completa [Joh67] com matriz de dissimilaridade que combina a correlação temporal e a distância física entre as séries temporais $\hat{\mathbf{s}}^{m}$. Isto permite obter grupos mais 
homogêneos e garante maior redução de dimensionalidade nas próximas etapas da LSCA.

O algoritmo de agrupamento começa com cada série temporal $\hat{\mathbf{s}}^{m}$ definindo um único grupo e, a cada passo, une os grupos $(A, B)$ que minimizam a seguinte função

$$
\operatorname{dist}(A, B)=\max \left\{\psi\left(\hat{\mathbf{s}}^{i}, \hat{\mathbf{s}}^{j}\right): i \in A, j \in B\right\}
$$

em que

$$
\psi\left(\hat{\mathbf{s}}^{i}, \hat{\mathbf{s}}^{j}\right)=\left\{\begin{array}{cl}
1, & \left|\bar{\phi}_{i}-\bar{\phi}_{j}\right|>\max \left(2^{l_{i}}, 2^{l_{j}}\right) \\
1-\left|\operatorname{cor}\left(\hat{\mathbf{s}}^{i}, \hat{\mathbf{s}}^{j}\right)\right|, & \text { caso contrário, }
\end{array}\right.
$$

em que $\operatorname{cor}\left(\hat{\mathbf{s}}^{i}, \hat{\mathbf{s}}^{j}\right)$ denota a correlação entre $\hat{\mathbf{s}}^{i}$ e $\hat{\mathbf{s}}^{j}, \bar{\phi}_{i}=\int_{\mathbb{R}^{d}} s\left|\phi_{i}\right|^{2} d s / \int_{\mathbb{R}^{d}}\left|\phi_{i}\right|^{2} d s$ define o centro de massa de $\phi_{i}$ e $l_{i}$ é o índice da escala de $\phi_{i}$ na decomposição WAVELETS. Assim, a medida de dissimilaridade acima combina a correlação e a distância física entre os coeficientes WAVELETS. O agrupamento acaba quando a distância mínima entre dois grupos é maior do que $r$ (i.e. $\min \{\operatorname{dist}(A, B): \forall A, \forall B\}>r$ ), para algum valor de $r$ escolhido apropriadamente, conforme discutido a seguir.

\section{Critério de Parada}

Embora a matriz de dissimilaridade (2.18) já limite bastante a estrutura dos componentes espaciais em (2.21), o critério de parada do algoritmo de agrupamento ainda precisa ser definido. Note que a medida de distancia $\operatorname{dist}(A, B)$ depende somente da correlação entre os coeficientes WAVElets em $A$ e $B$. Portanto, sob a hipótese nula, sua distribuição assintótica depende da transformada $\mathrm{z}$ de Fisher do coeficiente de correlação $0.5 \log _{e}\left(\frac{1+r}{1-r}\right)$ entre os elementos de ambos os grupos. A transformada z de Fisher do coeficiente de correlação possui distribuição conhecida, cujo limite superior para um intervalo com $(1-\alpha / 2) \%$ de confiança sobre a hipótese nula é aproximadamente dado por:

$$
u=z_{(1-\alpha / 2)} \sqrt{1 /(N-3)}
$$


em que $z_{(1-\alpha / 2)}$ é o $(1-\alpha / 2) \%$ percentil da distribuição normal padrão [Ste80]. Portanto nosso critério de parada é dado pelo seguinte valor

$$
r=1-|(\exp (2 u)+1) /(\exp (2 u)-1)|
$$

Note que o critério de parada não depende nem do nível de ruído ou do número de variáveis, apenas do tamanho amostral e do nível de significância $\alpha$ adotado. Neste trabalho, adota-se $\alpha=0.05$.

\subsubsection{Redução de Dimensionalidade}

O próximo passo consiste em estimar a matriz de observação $\mathbf{A}$ e o conjunto de fontes $\mathbf{x}_{t}$ utilizando a informação de cada grupo identificado no passo anterior. Após o agrupamento com restrição de contiguidade das linhas de $\hat{\mathbf{S}}$, o $k$-ésimo componente espacial $\mathbf{a}_{k} \mathbf{x}^{k}$ pode ser aproximado por

$$
\mathbf{Y}_{k}=\sum_{i \in I_{k}} \phi_{i}^{-1} \hat{\mathbf{s}}^{i}
$$

em que $\mathbf{Y}_{k}$ é uma matriz de dados de dimensão $M \times T, \phi_{i}^{-1}$ é a $i$-ésima coluna da matriz inversa de $\boldsymbol{\Phi}$ ( $\boldsymbol{\Phi}^{\mathrm{T}}$, para transformadas ortogonais) e $I_{k}$ contém os índices do $k$-ésimo grupo. Suponha que as linhas de $\mathbf{Y}_{k}$ tenham média zero, do contrário seu valor médio pode ser removido após (2.21).

De acordo com o modelo de aproximação

$$
\mathbf{Y}_{k}=\mathbf{a}_{k} \mathbf{x}^{k}+\mathbf{E}_{k}
$$

em que $\mathbf{E}_{k}$ é uma matriz de dimensão $M \times T$ que representa o erro de aproximação, o objetivo é encontrar $\mathbf{a}_{k}$ e $\mathbf{x}^{k}$ que minimiza o erro de aproximação

$$
\min _{\mathbf{a}_{k}, \mathbf{x}^{k}}\left\|\mathbf{Y}_{k}-\mathbf{a}_{k} \mathbf{x}^{k}\right\|_{F}
$$

em que $\|\cdot\|_{F}$ denota a norma de Frobenius [Mey00].

De fato, cada componente espacial $\mathbf{a}_{k} \mathbf{x}^{k}$ é uma matriz de dimensão $M \times T$ e posto unitário 
que pode ser obtida pelo primeiro valor singular de $\mathbf{Y}_{k}$, i.e:

$$
\mathbf{Y}_{k} \approx \sigma_{1} \mathbf{u}_{1} \mathbf{v}_{1}^{\mathrm{T}}
$$

em que $\sigma_{1}$ é o maior valor singular de $\mathbf{Y}_{k}$, e em que $\mathbf{u}_{1}$ e $\mathbf{v}_{1}$ são respectivamente o vetor singular esquerdo e o vetor singular direito associados a $\sigma_{1}$ [GR70]. Assim, sem perda de generalidade, pois $\left(\left\|\mathbf{a}_{k}\right\|^{2}=1\right)$, obtém-se o seguinte resultado:

$$
\begin{array}{r}
\mathbf{a}_{k}=\mathbf{u}_{1} \\
\mathbf{x}^{k}=\sigma_{1} \mathbf{v}_{1}^{\mathrm{T}} .
\end{array}
$$

\subsection{Dados Simulados}

\subsubsection{Modelo 1}

Para avaliar a performance da LSCA na detecção de fontes espacialmente bem localizadas, imagens bidimensionais representadas por $M=64 \times 64$ pontos e resolução $\Delta s=0.30$ foram geradas segundo o modelo (1.1) em que $\mathbf{x}_{t}$ representa um par de fontes correlacionadas, coeficiente de correlação igual à 0.5 , cuja distância relativa $\delta$ e o nível de ruído aditivo é variável. Uma função gaussiana bivariada com centro em $\boldsymbol{\mu}_{1}=[27+\delta, 27+\delta]$ e variância $\boldsymbol{\Sigma}_{1}=3 \mathbf{I}_{2}$, em que $\mathbf{I}_{2}$ é a matriz identidade de dimensão $2 \times 2$, foi utilizada como função de espalhamento para a primeira fonte. Na segunda fonte, utilizou-se uma função gaussiana bivariada anisotrópica centrada em $\boldsymbol{\mu}_{2}=[37-\delta, 37-\delta]$ e com variância $\boldsymbol{\Sigma}_{2}=\left[\begin{array}{cc}9 & 0 \\ 0 & 1\end{array}\right]$.

A transformada esparsa foi obtida por uma decomposição por pacotes WAVELETS com três níveis (veja 2.1.1) utilizando WAVELETs de Haar bidimensionais. O parâmetro de regularização $\lambda$ foi escolhido com nível de significância $\alpha=5 \%$. As fontes foram geradas como variáveis aleatórias independentes com média zero e variância unitária. Para avaliar a capacidade de reconstrução da LSCA, utilizou-se o valor de correlação médio entre as fontes simuladas $x_{k, t}$ e as fontes reconstruídas $\hat{x}_{k, t}$ ao longo de 30 repetições para cada combinação de $\delta=1,3$ e 5 e SNR $=-20,-18,-16,-14,-12,-10,-8,-6,-4$ e -2 . Os resultados fo- 
ram comparados com a análise PCA utilizando o valor máximo de correlação entre as fontes simuladas e os dois primeiros componentes principais para cada combinação de $\delta$ e SNR.

\subsubsection{Modelo 2}

Para avaliar a performance da LSCA na reconstrução de fontes dinâmicas espacialmente bem localizadas, séries temporais de tempo discreto foram amostradas em um espaço unidimensional representado por $M=256$ pontos espaciais cuja atividade evolui durante $T=500$ instantes de tempo. A Figura 2.2 (a) mostra a matriz de observação do modelo simulado dado por:

$$
\mathbf{A}=\left[\mathbf{f}_{80} \mathbf{f}_{180} \mathbf{f}_{100}\right]
$$

em que $\mathbf{f}_{\mu}=\left[f_{1, \mu}, \ldots, f_{M, \mu}\right]^{\mathrm{T}} \operatorname{com} f_{i, \mu}=f(i-\mu)$ e $f$ segundo uma função de espalhamento Gaussiana discreta. As observações foram corrompidas por ruído branco com distribuição Gaussiana com a seguinte matriz de covariância:

$$
\mathbf{R}=\sigma^{2} \mathbf{I}_{128,128}
$$

com $\sigma^{2}$ representando o nível de ruído $\mathrm{SNR}=10 \log _{10}\left(\operatorname{var}(\mathbf{s}) / \sigma^{2}\right)$, no qual $\mathbf{s}=\operatorname{vec}\left(\left[\mathbf{A x}_{1} \cdots \mathbf{A x}_{N}\right]\right)$. A dinâmica dos componentes espaciais evolui de acordo com um modelo autoregressivo de primeira ordem em que

$$
\mathbf{H}=\left[\begin{array}{ccc}
.5 & -.5 & 0 \\
0 & .5 & 0 \\
0 & 0 & 0
\end{array}\right]
$$

e

$$
\mathbf{Q}=\left[\begin{array}{lll}
1 & .5 & 0 \\
.5 & 2 & 0 \\
0 & 0 & 2
\end{array}\right]
$$

A Figura 2.2 (b) mostra a variância amostral de uma realização do modelo acima sob uma $\mathrm{SNR}=-19 \mathrm{db}$.

Os dados foram transformados utilizando uma decomposição WAVELET com função escala de Daubechies (D2) e a performance do algoritmo foi estimada por 100 simulações de Monte 


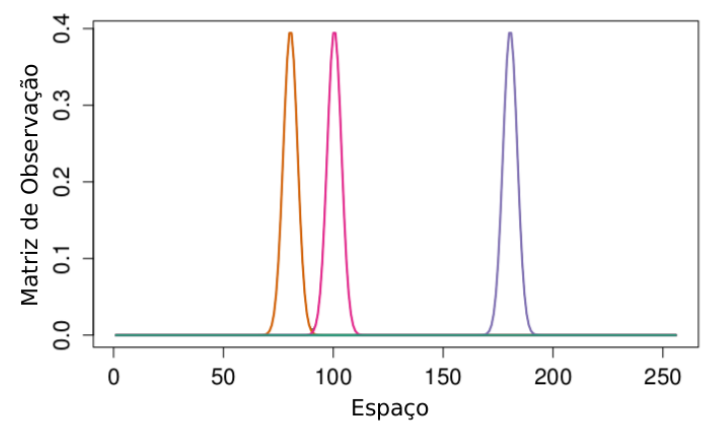

a

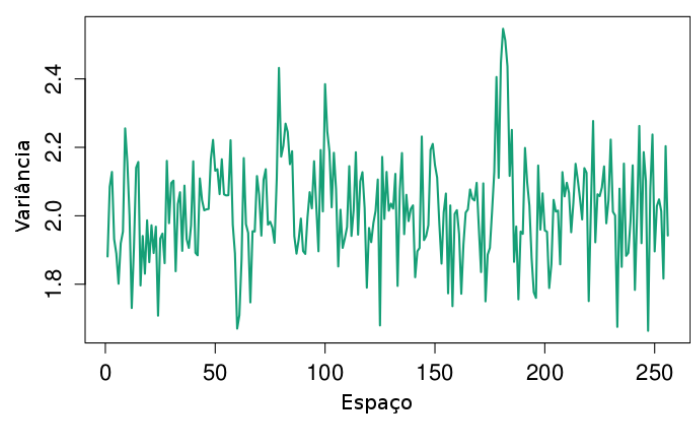

$\mathrm{b}$

Figura 2.2: (a) Representação gráfica das colunas da matriz de observação A do Modelo 2 (b) Variância amostral do Modelo 2 para $N=500$ e SNR $=-19 d b$.

Carlo. A efetividade do algoritmo foi avaliada de duas formas: (i) em termos da qualidade da reconstrução das fontes, dada pelo valor absoluto do coeficiente de correlação da fonte estimada com a fonte simulada; (ii) em termos da qualidade dos estimadores de $\mathbf{H}$ e $\mathbf{Q}$, dada pela diferença entre a Coerência Parcial Direcionada (PDC) [BDBTS13] estimada utilizando as fontes reconstruídas e a PDC utilizando as fontes simuladas. A função PDC foi utilizada como parâmetro de comparação pois, além de ser uma medida que leva em conta tanto a informação de $\mathbf{H}$ quanto a informação de $\mathbf{Q}$ no seu cálculo, o PDC é bastante utilizado em estudos de conectividade cerebral.

\subsection{Dados Reais}

Imagens de ressonância magnética de 11 voluntários sadios foram adquiridas em um equipamento de 3T Siemens Magnetom Trio utilizando uma sequência de aquisição de imagens EPI multiplexada (fator de aceleração de 4) $\left[\mathrm{FMS}^{+}\right.$10]. O estudo foi aprovado pelo comitê de ética local do Hospital Israelita Albert Einstein, São Paulo, SP e todos os participantes assinaram um termo de consentimento livre e esclarecido.

\subsubsection{Detalhes de Aquisição}

Os seguintes parâmetros foram utilizados na aquisição: TR $=600 \mathrm{~ms}$, TE $=33 \mathrm{~ms}, 32$ fatias, $\mathrm{FOV}=247 \times 247 \mathrm{~mm}$, tamanho matricial $128 \times 128$, resolução no plano $1,975 \times 1,975$ mm, espessura da fatia 3,5 mm com 1,8 mm de espaçamento. Para localização espacial das 
imagens de FMRI, uma imagem de alta resolução ponderada em T1 também foi coletada utilizando uma sequência MPRAGE com parâmetros: $\mathrm{TR}=2500 \mathrm{~ms}$, TE $=3,45 \mathrm{~ms}$, tempo de inversão $=1000 \mathrm{~ms}, 256 \times 256 \mathrm{~mm}$ FOV, $256 \times 256$ matriz no plano, $1 \times 1 \times 1 \mathrm{~mm}$ tamanho do voxel, $7^{\circ}$ ângulo de rotação).

\subsubsection{Processamento dos Dados}

\section{Parâmetros de Processamento da ICA}

A ICA foi realizado utilizando o método de concatenação entre múltiplas sessões implementado pela rotina MELODIC do pacote computacional $\mathrm{FSL}^{1}$, versão 5.0.6. O préprocessamento incluiu: (i) correção entre fatias, correção de movimento, remoção de crânio, filtro espacial (FWHM igual à 5mm) e filtro temporal passa alto (permitindo flutuações acima de $0.005 \mathrm{~Hz}$ ). As imagens funcionais foram alinhadas no espaço padrão MNI152 utilizando uma transformação linear com 12 graus de liberdade e suas séries temporais foram normalizadas para ter variância igual a um. O número de componentes foi fixado em 30 para obter componentes que coincidam com os padrões espaciais usualmente reportados por outros autores em estudos do estado de repouso $\left[\mathrm{DRB}^{+} 06, \mathrm{BMZ}^{+} 10\right]$.

\section{Parâmetros de Processamento da LSCA}

O pré-processamento da LSCA faz uso dos pacotes $\mathrm{AFNI}^{2}$, data de compilação: 5 de Dezembro de 2013, e FSL, versão 5.0.6. Após descartar os cinco primeiros volumes, as seguintes rotinas foram aplicadas: correção entre fatias pela função SLICETIME do FSL e correção de movimento pela 3DVOLREG do AFNI. As imagens funcionais foram suavizadas temporalmente para limitar valores extremos pela função 3DDESPIKE do AFNI, normalizadas para ter sua média global igual a 10.000 e alinhadas no espaço padrão MNI152 com 3mm de resolução utilizando uma transformação linear com 12 graus de liberdade pela função FLIRT do FSL.

Variáveis confundidoras foram removidas das imagens funcionais pela técnica de regressão múltipla. Além dos seis parâmetros de movimento calculados durante a correção de movi-

\footnotetext{
${ }^{1}$ www.fmrib.ox.ac.uk

2 afni.nimh.nih.gov/afni
} 
mento (translação e rotação nos três eixos), as séries temporais de cada indivíduo foram projetadas no espaço ortogonal dos sinais determinados pela média do sinal de FMRI nas seguintes regiões: substância branca, líquido cefalorraquidiano e cérebro. Esse último corresponde aos voxels não nulos obtidos após a remoção do crânio. As máscaras da substância branca e do líquido cefalorraquidiano foram obtidas a partir das imagens de alta resolução de cada participante utilizando o programa de segmentação automática FAST do FSL. 


\section{Capítulo 3}

\section{Resultados}

Este capítulo apresenta os resultados da aplicação da LSCA em diferentes cenários, avaliando sua performance na reconstrução de fontes em diferentes modelos comumente encontrados na literatura de FMRI.

\subsection{Simulações}

\subsubsection{Modelo 1}

Este modelo avalia a capacidade de reconstrução de fontes localmente esparsas com função de espalhamento gaussiana e sob efeito de ruído aditivo gaussiano. Comumente, PCA é a técnica utilizada nesse caso, pois garante reconstrução ótima das fontes em termos do erro quadrático médio.

A figura 3fig:simulation mostra a superioridade da LSCA em relação a PCA para $N=250$ e para diferentes níveis de ruído $\left(\sigma^{2}=1,3,5,7,9\right)$ que corresponde à $\mathrm{SNR}=-2.5,-7.5,-12.5,-17.5$ $\mathrm{e}-22.5 \mathrm{db}\left(\mathrm{SNR}=10 \log _{10}\left(\operatorname{VAR}(\mathbf{s}) / \sigma^{2}\right) \operatorname{com} \mathbf{s}=\operatorname{vec}\left(\left[\mathbf{A x}_{1} \cdots \mathbf{A x}_{N}\right]\right)\right.$, sugerindo que a LSCA supera a PCA na recuperação de fontes espacialmente locais mesmo sob uma relação sinal ruído muito baixa.

\subsubsection{Modelo 2}

Este modelo avalia a performance da LSCA na reconstrução de fontes dinâmicas espacialmente locais. Em todos os casos, utilizou-se o algoritmo EM para estimar os parâmetros do 

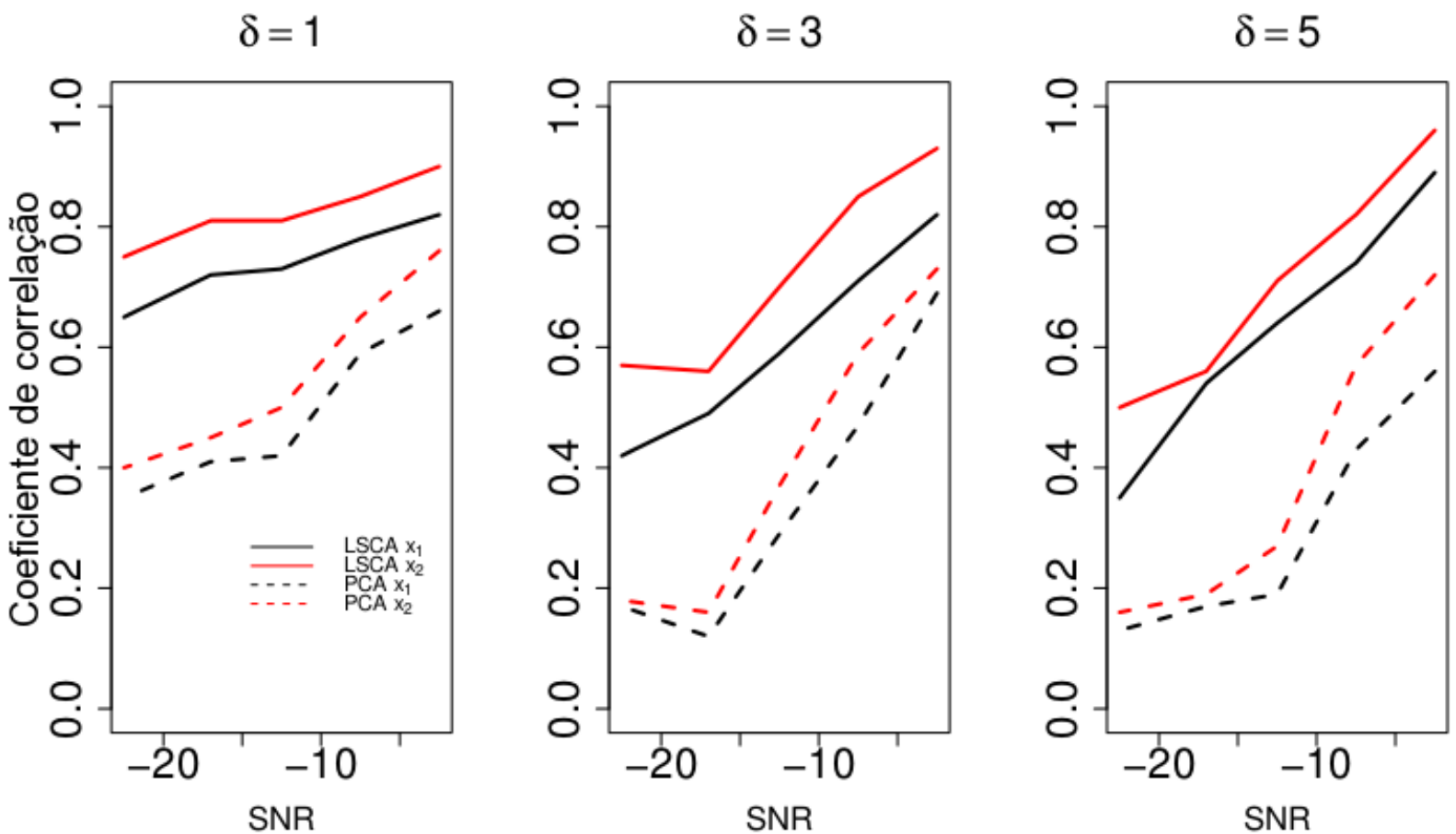

Figura 3.1: Comparação da eficiência das técnicas LSCA e PCA em reconstruir a informação temporal das fontes. As linhas representam a correlação média entre as fontes simuladas $x_{k, t}$ e as fontes estimadas $\hat{x}_{k, t}$ em 30 simulações.

modelos $(1.9,1.10)$. Entretanto, as estimativas obtidas utilizando a inicialização pela LSCA foram comparadas com as estimativas utilizando inicialização aleatória.

A Figura 3.2 mostra o valor absoluto médio do coeficiente de correlação entre as fontes simuladas e as fontes reconstruídas em função da SNR. É importante notar que a inicialização pela LSCA é melhor que a inicialização aleatória na reconstrução de todas as fontes e mesmo sob uma SNR muito desfavorável. A Figura 3.3 mostra o estimador de PDC entre as fontes $\mathbf{x}_{2}$ e $\mathbf{x}_{1}$ para diferentes níveis de SNR utilizando os diferentes tipos de inicialização. Observase que o padrão da curva de PDC é recuperado enquanto que a magnitude do estimador decai na medida em que a relação sinal ruído diminui.

\subsection{LSCA versus ICA}

Esta análise apresenta resultados da análise de dados de FMRI durante o estado de repouso utilizando a LSCA em comparação a ICA. O interesse desse estudo é demonstrar que a LSCA permite detectar as mesmas regiões comumente descritas em estudos do estado de repouso utilizando a ICA, sugerindo que a hipótese de independência não é uma hipótese 

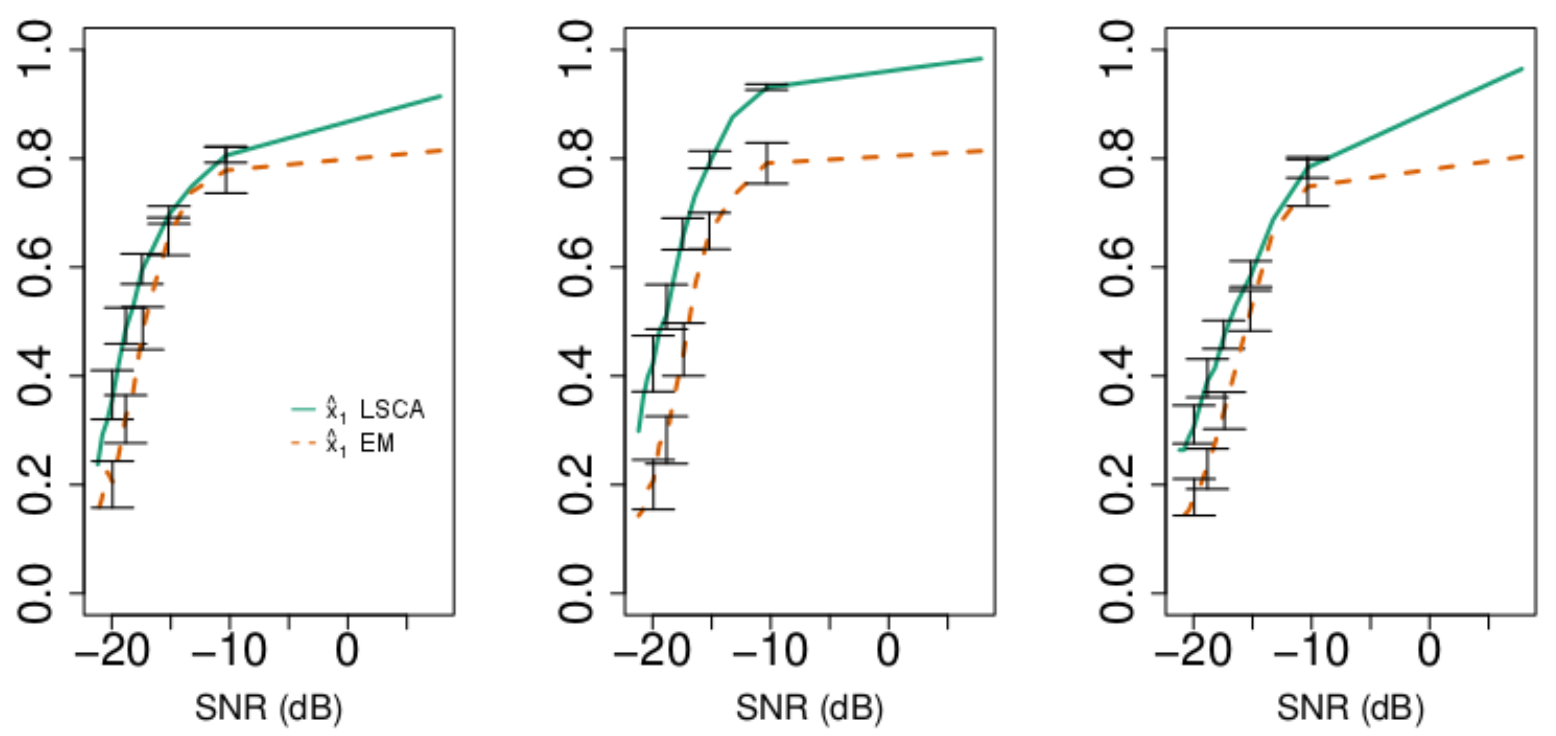

Figura 3.2: Comparação da eficiência da LSCA e EM em reconstruir a informação temporal das fontes. As linhas representam a correlação média entre as fontes simuladas $x_{k, t}$ e as fontes estimadas $\hat{x}_{k, t}$ em 100 simulações. Barras verticais representam o erro padrão.

necessária para solucionar o problema de separação cega em FMRI durante o estado de repouso.

\subsubsection{Resultados da ICA}

A figura 3.4 mostra 30 componentes obtidos pela ICA. Os componentes estão ordenados de acordo com a porcentagem relativa de variância explicada. Juntos, esses componentes representam $50 \%$ de toda variabilidade observada. Catorze componentes foram considerados artefatos fisiológicos por se concentrar preponderantemente no líquido cefalorraquidiano e substância branca (IC15, IC18, IC20, IC23, IC24, IC30), movimento de cabeça (IC17, IC22, IC26, IC29), e veias (IC09, IC13, IC14, IC28). Catorze foram identificados como componentes típicos do estado de repouso, compreendendo regiões da rede de modo padrão (IC02, IC10, IC11) e regiões envolvidas no processamento visual (IC01, IC04), auditivo/motor (IC05), sensorial/motor (IC08), atenção (IC03, IC06, IC16, IC19) e funções executivas (IC07, IC12, IC21). Além disso, encontramos dois componentes não típicos em estudos do estado de repouso. Um desses componentes está localizado no cerebelo (IC27) e o outro no tronco encefálico (IC25). 
SNR $=-10.32 \mathrm{~dB}$

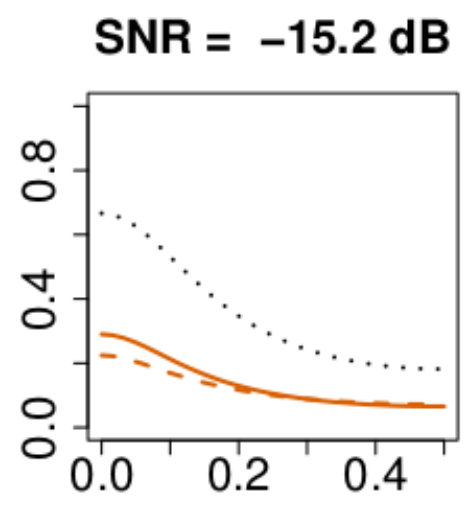

SNR $=-17.37 \mathrm{~dB}$
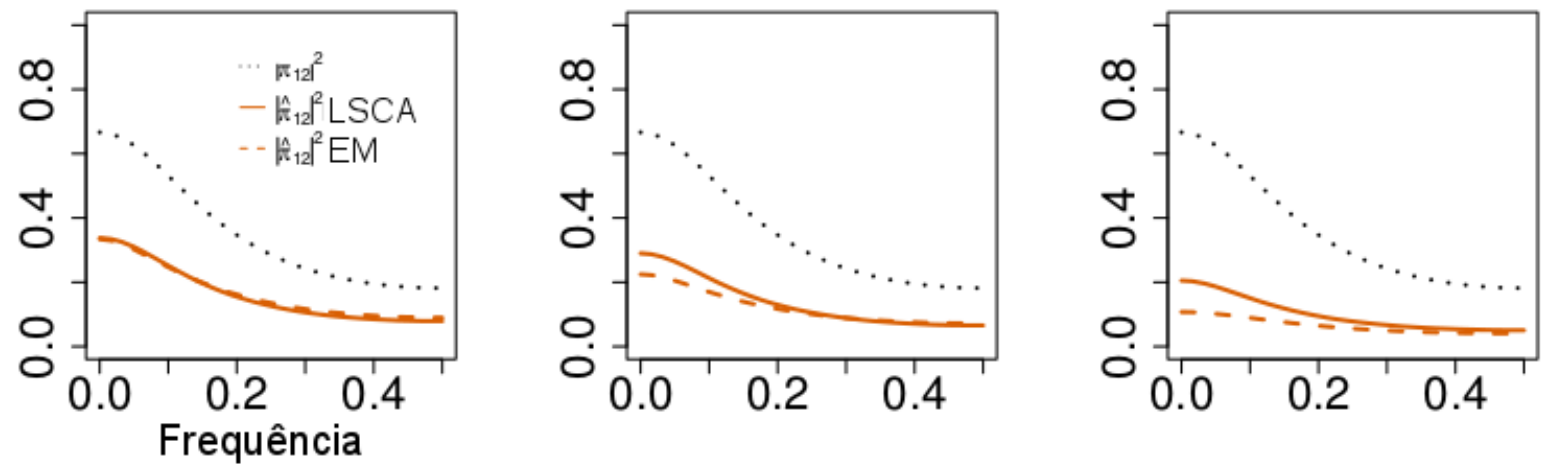

SNR $=-18.82 \mathrm{~dB}$
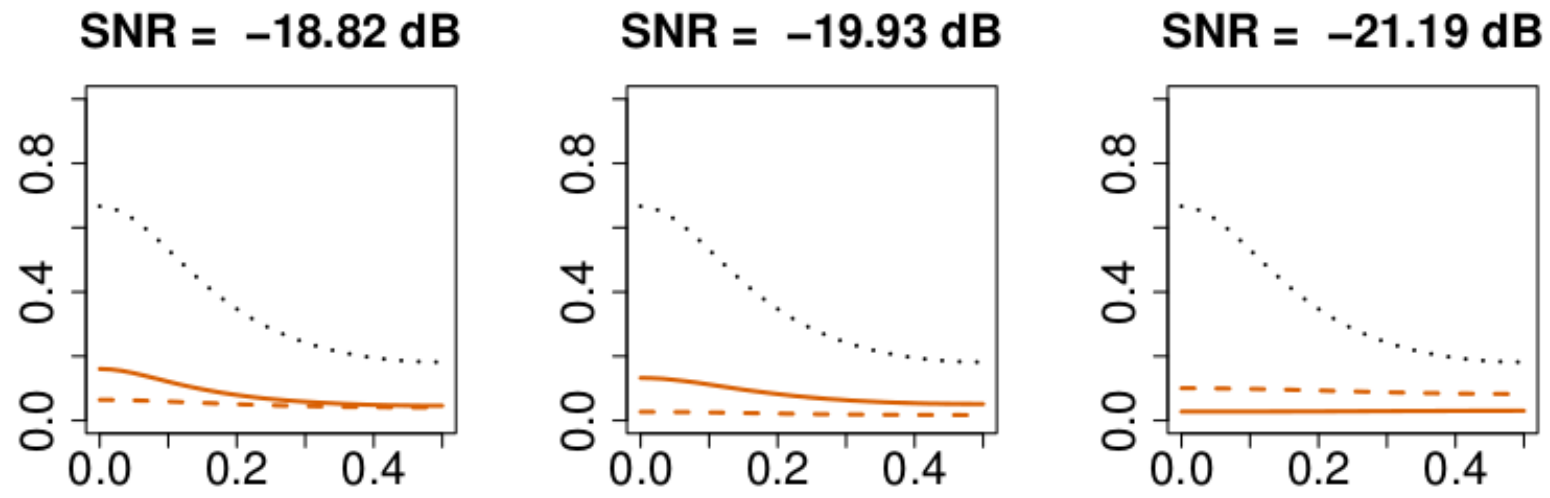

Figura 3.3: Linhas pontilhadas representam o valor teórico de PDC de acordo com o Modelo 2. Linhas sólidas representam o valor estimado de PDC utilizando o algoritmo EM com inicialização via LSCA. Linhas tracejadas representam o valor estimado de PDC utilizando o algoritmo EM com inicialização aleatória.

\subsubsection{Resultados da LSCA}

A Figura 3.5 ilustra como a transformada WAVELETS oferece uma representação muito mais compacta para imagens de FMRI: a entropia variância dos coeficientes no domínio WAVELETS é muito menor que a entropia da variância no domínio da imagem, ou seja, no domínio dos voxels. Isto significa que é possível representar grande parte da variabilidade temporal das imagens de FMRI utilizando um número reduzido de coeficientes WAVELETS. Por exemplo, somente 10\% dos coeficientes WAVELETS explicam $80 \%$ da energia total das imagens. Isso representa o dobro de energia que é explicada se utilizarmos $10 \%$ dos voxels com maior energia no domínio da imagem.

No total, a LSCA identificou 100 componentes. A tabela 3.1 apresenta os focos de cada componente ordenados de acordo com a porcentagem relativa de variância explicada. Os componentes são bem localizados de forma que a maioria deles se localiza somente em uma 


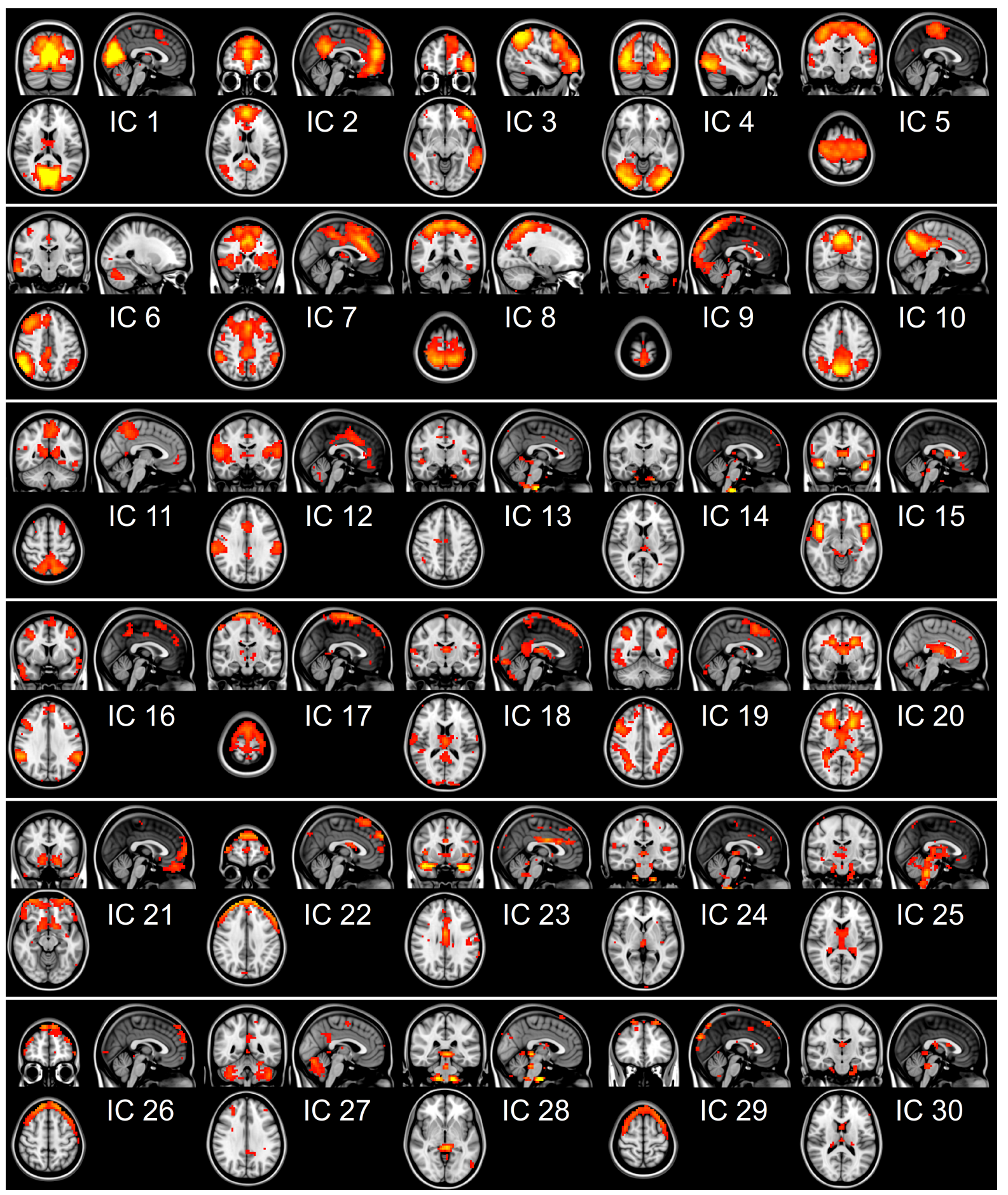

Figura 3.4: Componentes obtidos pela ICA. Os componentes estão ordenados de acordo com a porcentagem relativa de variância explicada. 
Tabela 3.1: Coordenadas MNI do máximo de atividade e nome anatômico dos componentes identificados pela $L S C A$.

\begin{tabular}{|c|c|c|c|c|c|c|c|c|c|}
\hline Cluster & $\mathbf{X}$ & $\mathbf{Y}$ & $\mathbf{Z}$ & Localização Anatômica & Cluster & $\mathbf{X}$ & $\mathbf{Y}$ & $\mathbf{Z}$ & Localização Anatômica \\
\hline 1 & -6 & -102 & 3 & Polo Occipital & 51 & -33 & -9 & 63 & Giro Pré-central \\
\hline 2 & 12 & -69 & 51 & Córtex Pré-cuneo & 52 & -33 & -78 & -45 & Cerebelo \\
\hline 3 & 9 & -81 & 27 & Córtex Cuneal & 53 & 39 & 15 & -21 & Polo Temporal \\
\hline 4 & -9 & -33 & 75 & Giro Pós-central & 54 & -57 & -9 & 27 & Giro Pós-central \\
\hline 5 & -33 & -93 & 3 & Polo Occipital & 55 & 9 & 63 & -18 & Polo Frontal \\
\hline 6 & 12 & 63 & 3 & Polo Frontal & 56 & 60 & -33 & 3 & Giro Temporal Superior \\
\hline 7 & 3 & 39 & 6 & Giro do Cíngulo & 57 & 39 & 63 & -9 & Polo Frontal \\
\hline 8 & 3 & -54 & 30 & Córtex Pré-cuneo & 58 & -54 & -33 & 51 & Giro Supramarginal \\
\hline 9 & 9 & -93 & -21 & Polo Occipital & 59 & -9 & -57 & -57 & Cerebelo \\
\hline 10 & -9 & -75 & 51 & Córtex Occipital Lateral & 60 & 39 & -69 & 51 & Córtex Occipital Lateral \\
\hline 11 & 6 & -57 & 66 & Córtex Pré-cuneo & 61 & 51 & 39 & -9 & Polo Frontal \\
\hline 12 & 3 & -33 & 75 & Giro Pré-central & 62 & 57 & -57 & 27 & Giro Angular \\
\hline 13 & -9 & 39 & 51 & Polo Frontal & 63 & -57 & -45 & 51 & Giro Angular \\
\hline 14 & 9 & -93 & 27 & Polo Occipital & 64 & 60 & -33 & 30 & Giro Supramarginal \\
\hline 15 & 0 & -81 & 3 & Córtex Intracalcarino & 65 & 39 & 51 & 3 & Polo Frontal \\
\hline 16 & 39 & -57 & 51 & Córtex Occipital Lateral & 66 & -48 & 39 & 3 & Polo Frontal \\
\hline 17 & -9 & 3 & 3 & Cerebelo & 67 & -33 & -81 & 42 & Córtex Occipital Lateral \\
\hline 18 & 51 & 39 & 3 & Polo Frontal & 68 & 3 & 39 & 51 & Giro Frontal Superior \\
\hline 19 & -6 & -33 & -21 & Tronco encefálico & 69 & 3 & 15 & 51 & Giro do Cíngulo \\
\hline 20 & -57 & -9 & 3 & Plano Temporal & 70 & -33 & 63 & -9 & Polo Frontal \\
\hline 21 & 3 & 15 & 27 & Giro do Cíngulo & 71 & 39 & 39 & 30 & Polo Frontal \\
\hline 22 & -33 & -57 & 57 & Córtex Occipital Lateral & 72 & 39 & -9 & 54 & Giro Pré-central \\
\hline 23 & 27 & -81 & 27 & Córtex Occipital Lateral & 73 & 3 & -66 & 51 & Córtex Pré-cuneo \\
\hline 24 & -9 & -33 & -66 & Cerebelo & 74 & -9 & -105 & 12 & Polo Occipital \\
\hline 25 & 15 & 63 & 27 & Polo Frontal & 75 & 3 & -9 & 69 & Lóbulo Temporal \\
\hline 26 & -45 & 39 & -21 & Polo Frontal & 76 & -57 & -33 & 27 & Córtex Opercular Parietal \\
\hline 27 & -9 & 63 & 27 & Polo Frontal & 77 & -9 & -69 & 57 & Córtex Pré-cuneo \\
\hline 28 & 0 & -57 & -9 & Cerebelo & 78 & -33 & 63 & 3 & Polo Frontal \\
\hline 29 & -9 & 15 & 51 & Giro Frontal Superior & 79 & -57 & -45 & 27 & Giro Angular \\
\hline 30 & 3 & -81 & 39 & Córtex Cuneal & 80 & 15 & 75 & 6 & Polo Frontal \\
\hline 31 & 3 & -93 & 27 & Polo Occipital & 81 & 9 & -81 & -9 & Giro Lingual \\
\hline 32 & 36 & -78 & -45 & Cerebelo & 82 & -45 & 39 & 27 & Polo Frontal \\
\hline 33 & 39 & 63 & 3 & Polo Frontal & 83 & 3 & -102 & 3 & Polo Occipital \\
\hline 34 & 48 & -57 & 27 & Giro Angular & 84 & -9 & -33 & -57 & Tronco encefálico \\
\hline 35 & 3 & -33 & 27 & Giro do Cíngulo & 85 & 39 & -21 & 51 & Giro Pré-central \\
\hline 36 & 51 & -45 & 51 & Giro Supramarginal & 86 & 0 & -21 & -51 & Tronco encefálico \\
\hline 37 & 60 & -9 & 3 & Plano Temporal & 87 & -3 & -57 & -63 & Cerebelo \\
\hline 38 & -9 & 15 & 63 & Giro Frontal Superior & 88 & -9 & -45 & 75 & Giro Pós-central \\
\hline 39 & -9 & -9 & 75 & Giro Frontal Superior & 89 & -39 & 15 & -21 & Polo Temporal \\
\hline 40 & -57 & -57 & 27 & Giro Angular & 90 & -57 & -57 & 3 & Giro Temporal Médio \\
\hline 41 & -33 & 15 & 51 & Giro Frontal Médio & 91 & 51 & 15 & 27 & Giro Frontal Inferior \\
\hline 42 & -33 & -81 & 27 & Córtex Occipital Lateral & 92 & -21 & 63 & -9 & Polo Frontal \\
\hline 43 & -33 & 51 & 3 & Polo Frontal & 93 & -21 & -93 & -21 & Polo Occipital \\
\hline 44 & -33 & -78 & -21 & Giro Fusiforme & 94 & -45 & 15 & -9 & Polo Temporal \\
\hline 45 & 39 & -81 & 27 & Córtex Occipital Lateral & 95 & 3 & -9 & 75 & Lóbulo Temporal \\
\hline 46 & -9 & -57 & -66 & Cerebelo & 96 & 60 & 15 & -18 & Polo Temporal \\
\hline 47 & -6 & 39 & -21 & Córtex Frontal Medial & 97 & 12 & -33 & -57 & Tronco encefálico \\
\hline 48 & 39 & -78 & -21 & Córtex Occipital Lateral & 98 & 60 & 15 & 3 & Giro Frontal Inferior \\
\hline 49 & 3 & 63 & 6 & Polo Frontal & 99 & -33 & 75 & 3 & Cerebelo \\
\hline 50 & 60 & -9 & 27 & Giro Pós-central & 100 & -36 & -45 & 51 & Giro Parietal Superior \\
\hline
\end{tabular}




\section{Curvas de Energia}

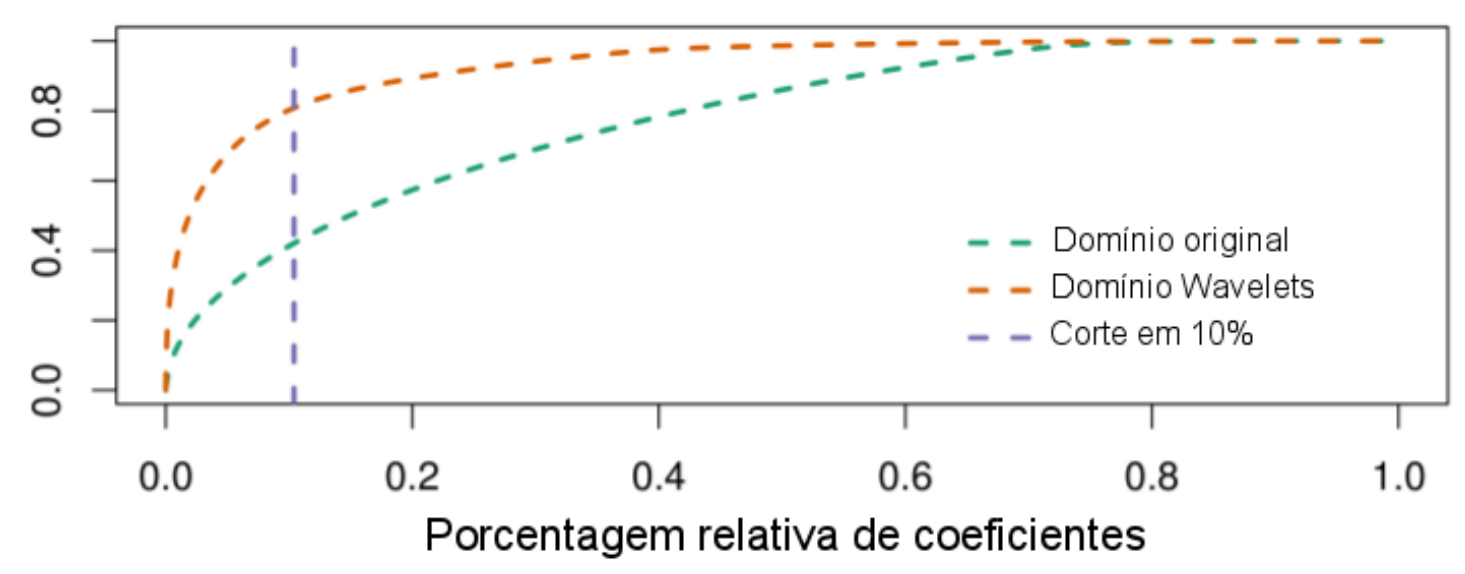

Figura 3.5: Fração de energia acumulada no domínio dos voxels (em verde) e no domínio WAVELETS (em vermelho) para o conjunto de dados de FMRI durante o estado de repouso. A linha vertical em azul indica a fração de energia representada por $10 \%$ dos coeficientes com maior energia no domínio dos voxels (40\%) e no domínio WAVELETS (80\%).

região anatômica específica, porém juntos eles cobrem grande parte das estruturas cerebrais e se sobrepõem com regiões típicas do estado de repouso.

A Figura 3.6 mostra 24 componentes da LSCA cujas fontes se correlacionam significantemente com pelo menos um dos seis componentes da ICA com maior variabilidade. É possível observar que cada componente da ICA pode ser representado por um conjunto de componentes da LSCA. Os componentes visuais da ICA, IC1 e IC4, estão fortemente associados com componentes da LSCA que estão localizados no córtex visual, incluindo as porções laterais e mediais. O segundo componente da ICA, IC2, contendo regiões que fazem parte da rede de modo padrão, são significantemente correlacionados em componentes esparsos localizados no cíngulo posterior e córtex pré-frontal medial. Finalmente, os primeiros quatro componentes da LSCA que mais se correlacionam com os componentes IC3 e IC6 da ICA componentes da rede frontoparietal esquerda e direita, respectivamente - estão localizados em regiões frontais e parietais dos hemisférios cerebrais esquerdo e direito, respectivamente. 


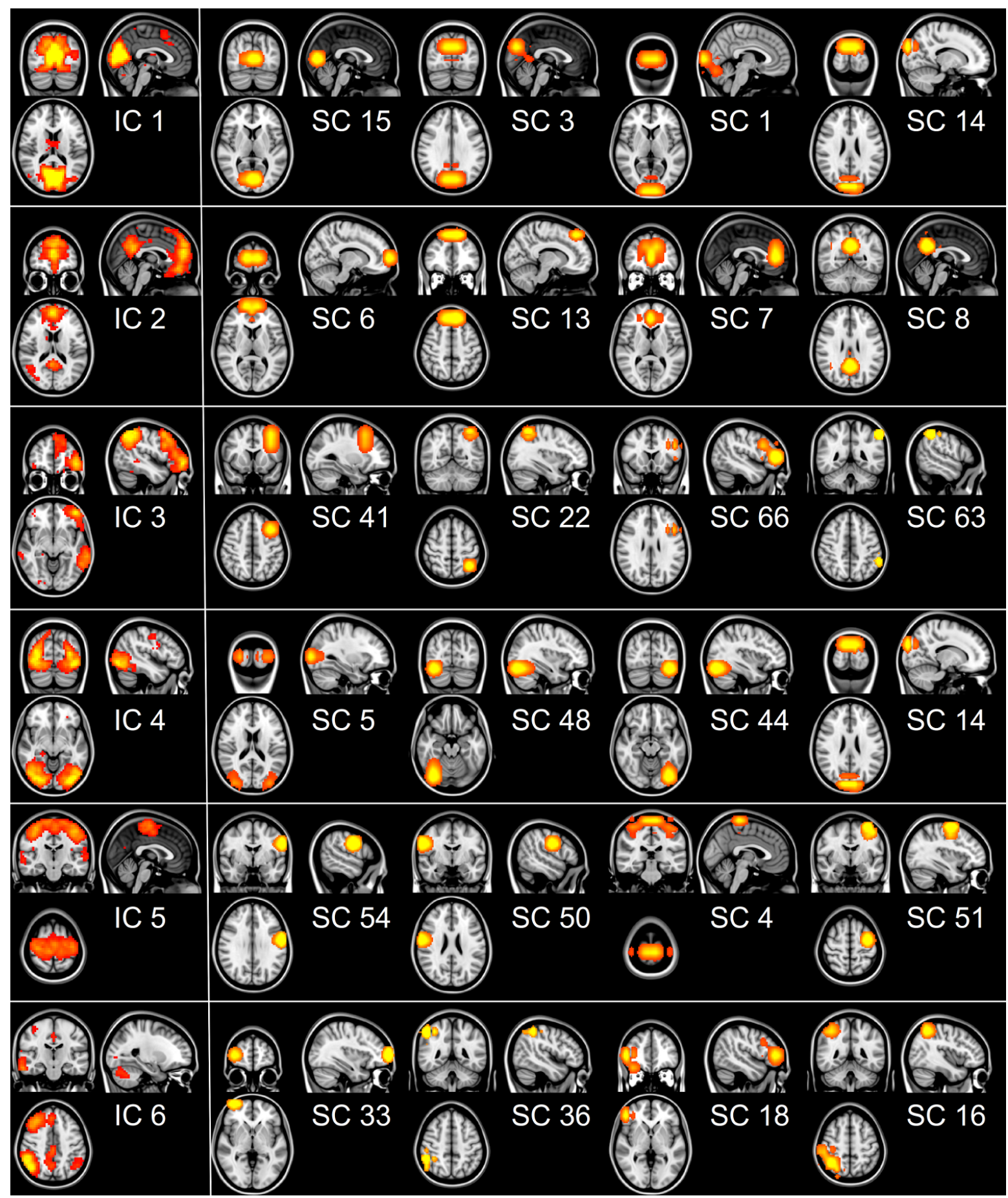

Figura 3.6: Componentes identificados pela LSCA (da segunda até a quinta coluna) cuja dinâmica está significativamente correlacionada com a dinâmica de pelo menos um dos seis componentes identificados pela ICA com maior variabilidade (primeira coluna). 


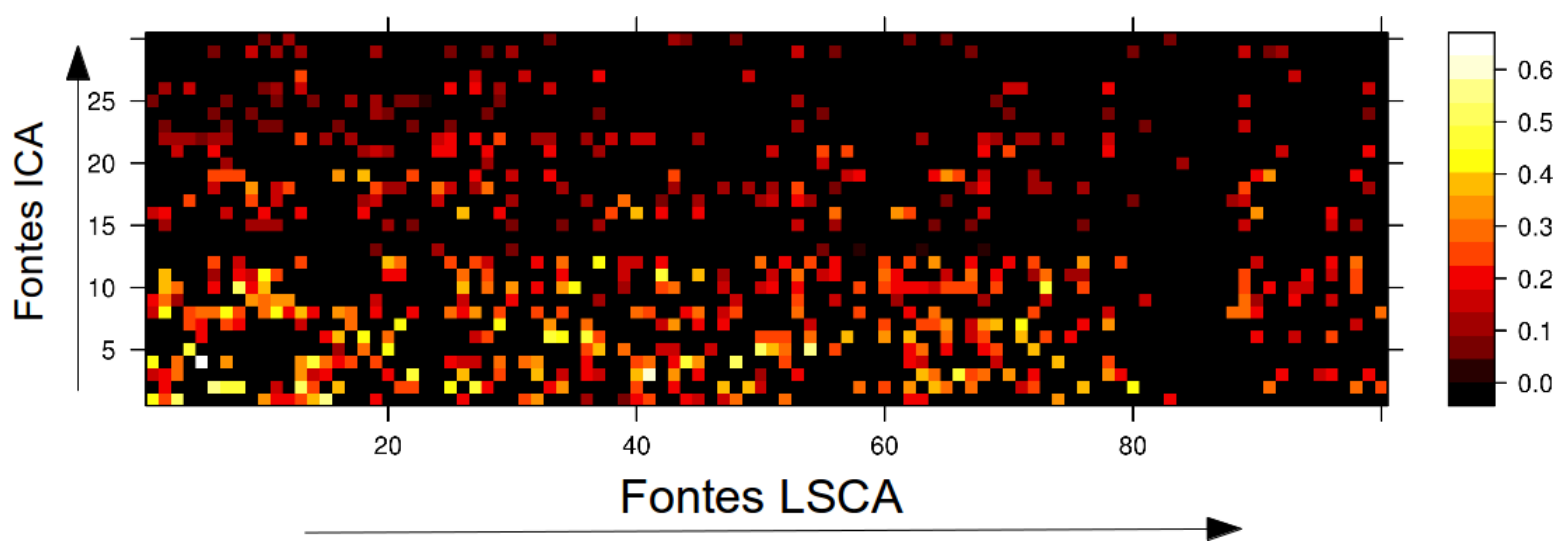

Figura 3.7: Correlação de Pearson entre as fontes identificadas pela LSCA e ICA (corrigido para múltiplas comparações via $F D R, q<0.05)$. A menos das fontes que correspondem a componentes ruidosos devido a grande vasos (IC14 e IC28), todas as fontes identificadas pela ICA se correlacionam com pelo menos uma fonte identificada pela LSCA.

\subsubsection{Comparação entre LSCA e ICA}

Aqui, a relação entre as fontes identificadas pela ICA e LSCA foi avaliada em termos do coeficiente de correlação de Pearson. Para cada par de fontes o coeficiente de correlação de Pearson médio entre os participantes foi estimado e sua significância foi corrigida para múltiplas comparações via FDR $(q<0.05)$. Os resultados estão apresentados na Figura 3.7 (A). Observa-se que grande parte das fontes obtidas pela ICA possuem correlação significativa com alguma fonte obtida pela LSCA. As únicas fontes obtidas pela ICA que não se correlacionam com nenhuma fonte obtida pela LSCA são fontes que correspondem a ruído devido a grandes vasos IC14 e IC28. Por outro lado, as fontes obtidas pela LSCA que não se correlacionam com nenhuma fonte obtida pela ICA estão espalhadas por todo o cérebro (LSC23, LSC28, LSC38, LSC46, LSC55, LSC59, LSC77, LSC84, LSC85, LSC86, LSC87, LSC100). A Figura 3.8 (D) mostra a correlação máxima entre cada fonte obtida pela ICA e as demais fontes obtidas pela LSCA. A semelhança entre as fontes é maior entre os componentes com maior variabilidade, e portanto, com maior relevância biológica. Além da componente localizada no tronco encefálico (IC25), as fontes obtidas pela ICA que não se correlacionam com nenhuma fonte obtida pela LSCA são fontes consideradas artefatos em estudos sobre o estado de repouso (IC13, IC14, IC20, IC23, IC24,IC28, IC30).

Por último, a relação entre as fontes estimadas e os sinais observados foi investigada por meio da correlação entre cada fonte estimada e a série temporal média dos voxels de seu respectivo componente espacial. Os resultados estão apresentados na Figura 3.8) (A) e (B). 


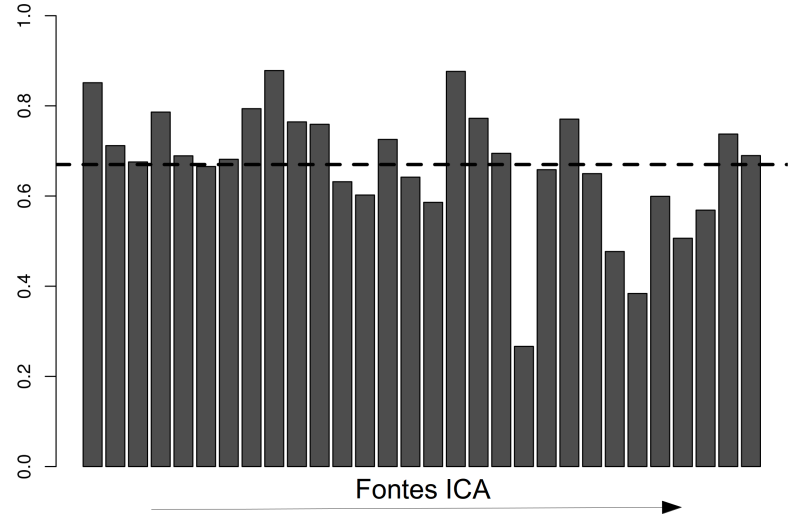

A

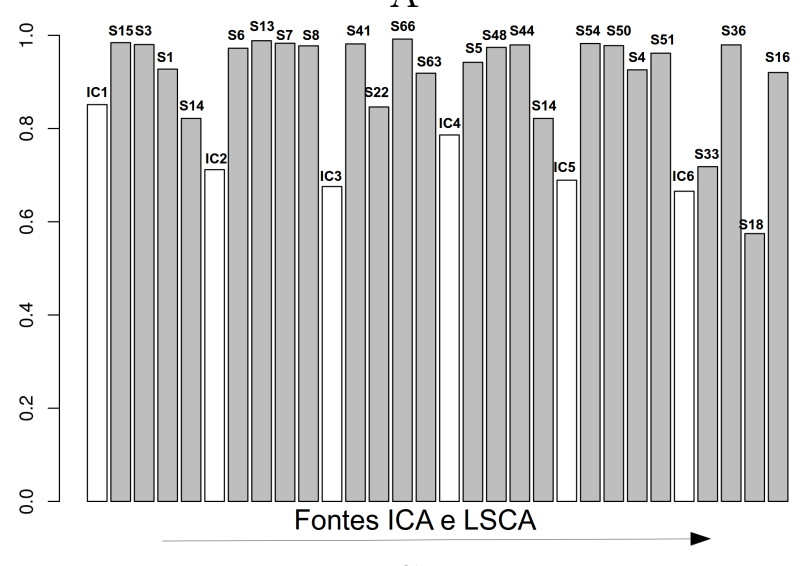

$\mathrm{C}$

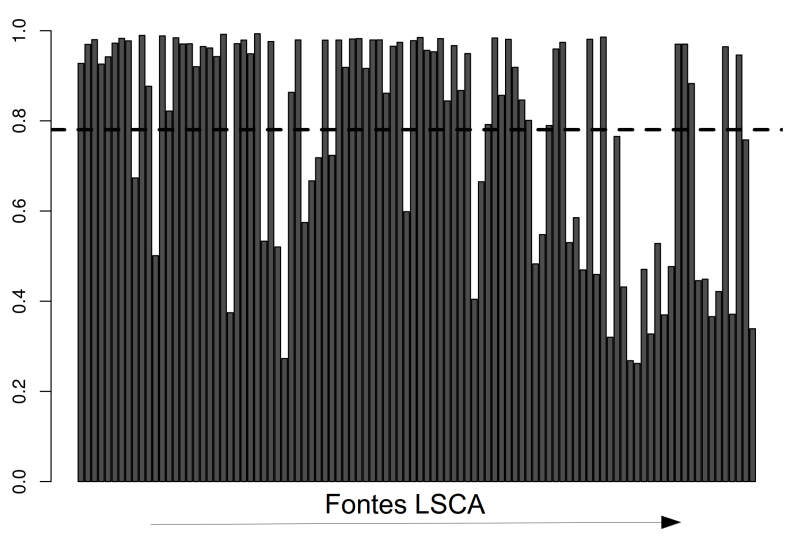

$\mathrm{B}$

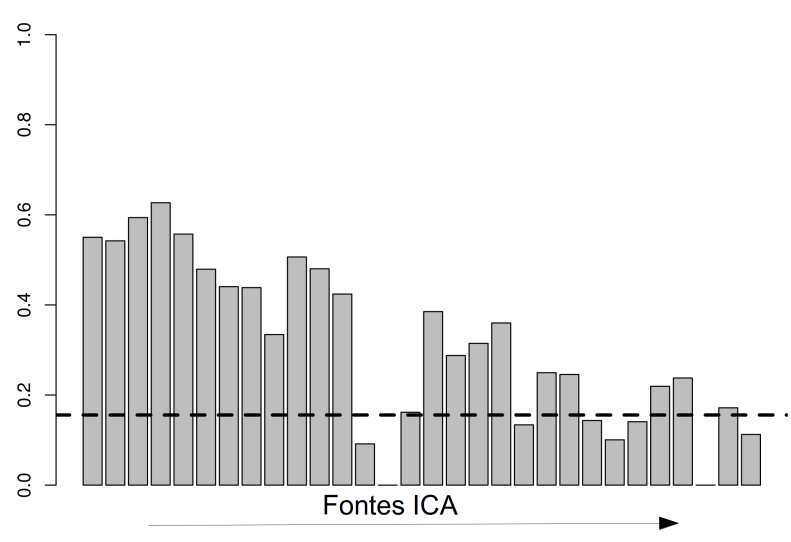

$\mathrm{D}$

Figura 3.8: Correlação entre cada fonte estimada e sua respectiva série temporal para as análises pela ICA (A) e LSCA (B). A correlação média ao longo dos participantes é maior entre as fontes identificadas pela LSCA do que entre as fontes identificadas pela ICA (LSCA: $r=0.78$; ICA: $r=0.67 ; p<0.001)$, sugerindo que a LSCA representa melhor a variabilidade dos dados que ICA. (C) Esta relação é ainda maior entre as fontes que representam as redes clássicas do estado de repouso. As correlações médias entre as fontes e suas respectivas séries temporais extraídas dos componentes da Figura 3.6 estão descritas em branco para a análise pela ICA e em cinza para a LSCA. (D) Valor de correlação máximo (eixo das y) entre cada fonte detectada pela ICA (eixo x) e todas fontes detectadas pela LSCA. 
Em média, fontes identificadas pela LSCA se correlacionam mais com sua respectiva série temporal que fontes identificadas pela LSCA (LSCA: $r=0.78$; ICA: $r=0.67 ; p<0.001$ ), sugerindo que a decomposição pela LSCA reflete mais os sinais observados. Novamente, a correlação é maior entre os componentes que possuem maior variância. A Figura 3.8 (C) mostra a correlação média entras fontes obtidas pela ICA e as fontes obtidas pela LSCA para cada componente da Figura 3.6.

\subsection{Análise de Componentes Dinâmicos Esparsos Locais}

Simulações numéricas de modelos dinâmicos com fontes localmente esparsas mostram que a inicialização do algoritmo EM utilizando a LSCA supera a abordagem clássica, baseada em inicialização aleatória, permitindo estimar suas fontes e parâmetros de forma aceitável mesmo sob condições desfavoráveis de relação sinal ruído. Portanto, esse estudo busca explorar as características dinâmicas das redes do estado de repouso através do algoritmo EM cuja inicialização é feita utilizando a LSCA. No apêndice C, o algoritmo EM é discutido em detalhes. É importante notar que, devido a alta dimensionalidade do problema que consiste em estimar todos os parâmetros do modelo $(1.9,1.10)$ em dados de FMRI, o algoritmo EM só funciona quando inicializado de maneira correta. Uma vez inicializado, o algoritmo consiste em aplicar as interações tradicionais do algoritmo EM [SS82].

A Figura 3.9 (a) resume a rede de conectividade obtida aplicando a técnica PDC as fontes reconstruídas. Além disso, a Figura 3.9 (b) destaca que a conectividade do estado de repouso estimada via PDC está presente principalmente em baixas frequências, corroborando com vários estudos de conectividade cerebral no estado de repouso $\left[\mathrm{BMZ}^{+} 10\right]$. 


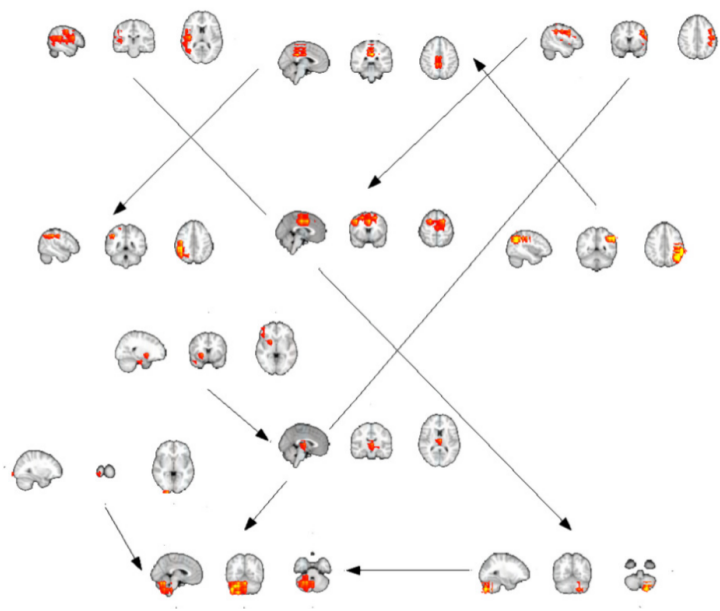

a
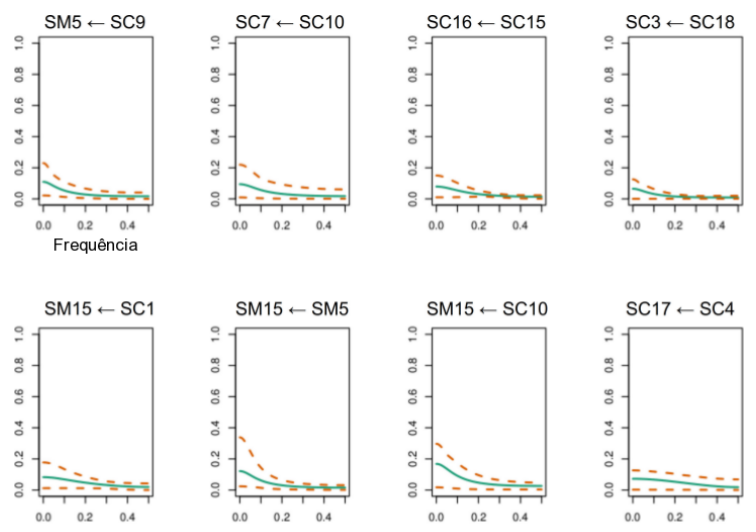

b

Figura 3.9: Análise da dinâmica dos componentes identificados através da LSCA. (a) Representação gráfica do fluxo de informação entre os componentes de acordo com a análise PDC das fontes. (b) Gráficos do valor de PDC entre as regiões ligadas por setas na Figura (a). Linhas tracejadas denotam o intervalo com $95 \%$ de confiança para o valor médio de PDC (linhas sólidas). 


\section{Capítulo 4}

\section{Discussão}

Representações esparsas e separação cega de fontes espacialmente locais com aplicação em FMRI são discutidas nesta tese. A LSCA é uma técnica que usa operadores de limiarização vetorial para obter esparsidade de grupo. Dada uma transformação esparsa baseada na transformada WAVELETS, identifica-se as fontes por um algoritmo de agrupamento que preserva contiguidade espacial e homogeneidade temporal entre grupos. Isso garante uma redução de dimensionalidade de maneira eficiente por meio de projeções ortogonais em subespaços unidimensionais. A LSCA é útil para separação cega de fontes esparsas locais, especialmente em análises de sistemas dinâmicos espaço-temporais com alta redundância espacial.

\subsection{Simulações}

Os modelos simulados nesta tese ilustram a performance da LSCA na identificação da localização e estimação da dinâmica de fontes esparsas locais. Os resultados destas simulações foram discutidos simultaneamente à apresentação dos mesmos.

\subsection{LSCA versus ICA}

Grande parte dos estudos de neuroimagem se concentra na ICA para estimar redes do estado de repouso. Esta tese demonstra que redes do estado de repouso também podem ser estimadas utilizando uma decomposição baseada na redundância espacial dos dados que 
garante componentes espacialmente locais. Por não impor restrições na extensão espacial de seus componentes, a ICA não garante localização exata das fontes. Além disso, fontes identificadas pela LSCA representam aproximações locais dos sinais e, portanto, refletem melhor o processamento local das regiões representadas pelos componentes. Porém, em estudos do estado de repouso, fontes identificadas pela LSCA se correlacionam fortemente com fontes identificadas pela ICA, um resultado que suporta a ideia de esparsidade em FMRI $\left[\mathrm{DRT}^{+}\right.$09]. Uma outra possível explicação para a similaridade entre os resultados obtidos pela ICA e a LSCA nesta tese se deve ao fato de que o pré-processamento da ICA realiza uma projeção ortogonal dos dados para reduzir a dimensionalidade das imagens baseada na PCA. Essa projeção poderia, a princípio, estar influenciando os resultados do ICA mais do que a própria operação de rotação utilizada para maximizar a independência entre as fontes.

Assim como foi observado na ICA, os componentes corticais da LSCA refletem, em grande parte, a variabilidade espacial de regiões contidas em redes do estado de repouso: DMN e rede visual, rede motor e rede fronto parietal, indicando que tais redes podem ser decompostas em componentes esparsos com localização e significado biológico válido (Figura. 3.4). Entretanto, os resultados apresentados nesta tese chamam atenção para o fato de que esses componentes foram identificados utilizando somente as premissas de esparsidade e localização das fontes. Isso corrobora a hipótese de que a efetividade da ICA em FMRI se deve à sua habilidade em tratar componentes esparsos em vez de componentes independentes [DRT ${ }^{+}$09].

Dos componentes identificados na LSCA, 8\% deles se encontram no cerebelo. Esse resultado é surpreendente, considerando que componentes cerebelares são dificilmente reportados em estudos do estado de repouso [BMZ+10]. Como LSCA utiliza um critério de seleção baseado na energia de seus componentes, é possível que os componentes cerebelares identificados nesta tese não tenham uma origem neuronal, mas representem variações do sinal de FMRI causado por artérias e veias próximas. É importante notar, entretanto, que disparidades entre a ICA e a LSCA podem ocorrer simplesmente pelas características do domínio de representação de seus componentes. Assim como acontece no córtex cerebral, muitos estudos de FMRI apontam para uma especialização funcional do cerebelo no processamento de funções de diferentes domínios cognitivos ([SS09] para uma revisão) onde as regiões cerebelares especializadas seriam menores que suas respectivas regiões corticais especializadas. Isso 
sugere que LSCA é mais específico na caracterização de componentes com baixa extensão espacial - um fato que já foi notado em [VAB14].

O pré-processamento aplicado na LSCA difere do pré-processamento aplicado na ICA em dois aspectos que visam melhorar a comparação entre as técnicas: uso de filtro espacial e remoção de variáveis confundidoras. Primeiro, embora o uso de filtros espaciais seja um procedimento padrão em FMRI por aumentar a relação sinal ruído das imagens, LSCA dispensa essa etapa, pois a transformada WAVELETS em múltiplas escalas representa um banco de filtros com filtros ortogonais de diferentes tamanhos. Isso significa que a LSCA permite aumentar a especificidade de seus componentes espaciais sem prejudicar a relação sinal ruído. Segundo, a remoção de variáveis confundidoras na LSCA por meio de regressão múltipla visa remover o efeito do movimento da cabeça e variações na substância branca, líquido encéfalo raquidiano e no cérebro de maneira global. Esse passo é desnecessário na ICA devido a sua capacidade singular de identificar tais componentes ruidosos [BU99].

Finalmente, é importante notar as principais questões envolvidas na escolha do número de componentes tanto na ICA quanto na LSCA. Embora existam técnicas para determinar automaticamente o número de componentes na ICA [BDDS05], na prática, esse número é escolhido baseado no conhecimento a priori do pesquisador. Um dos grandes problemas dessa abordagem é determinar a extensão espacial dos componentes baseado no número de componentes. Na prática, quanto mais componentes são identificados, menores eles são. A situação se agrava quando o aumento do número de componentes transforma regiões dependentes em independentes. Por outro lado, o número de componentes na LSCA depende somente de um parâmetro regularizador que representa o nível de esparsidade global dos dados. Embora o parâmetro de regularização seja, em última análise, definido pelo usuário, seu valor não afeta a extensão espacial dos componentes.

\subsection{Análise de Componentes Dinâmicos Esparsos Locais}

Como apresentado nesta tese, a LSCA é uma abordagem para localizar e estimar fontes em imagens de FMRI durante o estado de repouso que dispensa premissas artificiais comumente empregadas por métodos tradicionais [FFLF93, BS04, GTCB07, LVU07]). Além 
disso, a hipótese de esparsidade permite estimar a conectividade funcional no cérebro inteiro através de um modelo linear com formulação por espaço de estados.

A análise da conectividade revelou uma rede no qual a informação flui do córtex parietal superior para o cerebelo e cíngulo anterior. Como esperado, o córtex parietal superior direito envia informação para o cerebelo esquerdo, enquanto o córtex parietal superior esquerdo envia informação para o cerebelo direito. Embora a relação entre essas estruturas não seja bem compreendida ainda, a conectividade entre o córtex parietal superior e o cerebelo está de acordo com estudos recentes que mostram evidências de uma rede envolvendo essas duas regiões no armazenamento fonológico [MBVP14]. Além disso, o circuito envolvendo os córtices visual, parietal e cerebelar já foi estimado em outro estudo de conectividade em FMRI $\left[\mathrm{KRDV}^{+} 12\right]$. Também é possível observar uma rede que se inicia no córtex parietal esquerdo e se estende até o córtex parietal direito passando pelo cíngulo posterior. Juntos, os resultados da análise de conectividade indicam mecanismos de interação entre regiões da rede frontoparietal e, também, apontam aspectos ainda não explorados do papel do cerebelo nessa rede durante o estado de repouso.

\subsection{Considerações Finais}

A LSCA é um método de modelagem e redução de dimensionalidade que codifica a redundância espacial de imagens de FMRI de maneira eficiente e, portanto, permite abordar eficientemente vários problemas que surgem em estudos de conectividade funcional. Primeiramente, ao contrário da ICA, a LSCA permite análises mais profundas da conectividade entre fontes ao não assumir independência entre os componentes espaciais. Um outro problema recorrente que aparece na análise de conectividade está associado a localização espacial das regiões de interesse. Neste sentido, a LSCA permite localizar fontes de alta variabilidade que possuem características próprias de regiões de interesse. Isso significa que a LSCA é ideal para estudos de conectividade exploratórios que buscam caracterizar o cérebro por meio de técnicas de análise de redes e grafos [WZH10]. Finalmente, um outro problema bastante comum em estudos de conectividade é a necessidade de se constrir modelos para sistemas dinâmicos que envolvem muitas variáveis. Neste contexto, a LSCA permite decompor sistemas 
espaço-temporais em múltiplas escalas para criar um modelo compacto do sinal observado. 


\section{Capítulo 5}

\section{Conclusão}

No processamento de sinais de alta dimensão, o conceito de esparsidade surgiu para formalizar a noção de representação eficiente de dados. A descoberta de bases ortogonais bem localizadas no espaço e tempo lançou bases para um universo de novas transformações com a capacidade de decompor sinais complexos em poucos componentes elementares. Transformações esparsas se tornaram os blocos de construção em diversos problemas de processamento de sinais, como aproximação esparsa, compressão de dados, redução de dimensionalidade, remoção de ruído e solução de problemas inversos.

Este trabalho desenvolveu um novo método para separação cega de fontes localmente esparsas. O método proposto adota uma ideia similar a SCA, visando mapear os dados em um domínio onde as fontes possam ser facilmente obtidas utilizando um algoritmo de agrupamento. O novo método foi denominado LSCA e difere de outras técnicas de SCA por supor intuitivamente que as fontes são locais e, portanto, podem ser separadas por transformadas WAVELETS espaciais. Para uma análise quantitativa de performance, a LSCA foi comparada com técnicas tradicionais em diferentes contextos. A LSCA foi comparado com a PCA na deteç̧ão de fontes gaussianas esparsas locais e com o algoritmo EM na detecção de fontes dinâmicas esparsas locais. Em ambos os casos, o desempenho da LSCA foi superior.

Na prática, a LSCA é comparável com a ICA na detecção de fontes em imagens de FMRI no estado de repouso. Os estudos desta tese demonstram que a LSCA pode detectar os mesmos componentes biologicamente plausíveis comumente detectados no estado de re- 
pouso utilizando a ICA, porém utilizando somente a hipótese de esparsidade. Em especial, componentes identificados pela LSCA representam melhor as variações locais dos sinais de FMRI do que componentes identificados pela ICA. Além disso, ao contrário de formulações mais restritivas, a LSCA permite detectar componentes dinamicamente relacionados.

A LSCA pode ser desenvolvidada de várias maneiras, especialmente em relação a escolha do parâmetro de regularização que se relaciona com a quantidade de ruído presente nos dados. Na presente implementação, o ruído espacial é supostamente homogêneo e normalmente distribuído, o que implica uma distribuição chi-quadrado para a variância dos coeficientes WAVELETS (equação (2.10)). Entretanto, em dados reais de FMRI, a distruibuição da variância dos coeficientes WAVELETS sugere características típicas de distribuições mistas, principalmente para valores extremos (figura 3.5). Uma possível direção seria avaliar a presença de efeitos mistos na distribuição dos coeficientes WAVELETS.

Entretanto, LSCA possui algumas limitações inerentes da decomposição esparsa adotada, principalmente porque as transformações esparsas aqui utilizadas foram desenvolvidas utilizando filtros estacionários, ideais para domínios retangulares, ou seja, imagens constituídas de objetos com um padrão simples de continuidade. Tais transformações não são eficientes para caracterizar domínios arbitrários e convoluídos como o córtex cerebral. Felizmente, transformações esparsas mais flexíveis podem ser construídas para domínios arbitrários não retangulares utilizando o algoritmo LifTing SCHEme [Swe95, Swe96]. Ao contrário das transformações tradicionais, as funções do dicionário obtido pelo algoritmo LIFTING SCHEME são definidas por filtros não estacionários e, portanto, não representam translações e dilatações de uma função protótipo [DS98]. Mesmo assim, elas mantém muitas características atrativas das transformadas tradicionais, como a representação esparsa e localizada, e processamento eficiente [Swe98].

\subsection{Trabalhos Futuros}

Considere $z$ um sinal observado ao longo do espaço e tempo. Em geral, a localização dos coeficientes $p_{j}[n, p]$ e $u_{j}[n, p]$ do algoritmo LIFTING SCHEME é bastante complicada quando espaço e tempo são modelados conjuntamente [KPPBBP12, HPPP05, PPPHP06]. Entre- 
tanto, para dados onde espaço e tempo são aproximadamente independentes, o algoritmo LIFTING SCHEME possui uma descrição surpreendentemente compacta, pois a transformação pode ser computada para cada $t$. Isso acarreta em diversos desdobramentos interessantes que têm sido exploradas por outros autores [CJBN98, KPPBBP12, KBBPPP11, HPPP05, PPPHP06, Thi01]. Um consequência é que os coeficientes $p_{j}[n, p]$ e $u_{j}[n, p]$ podem ser estimados utilizando os próprios dados.

A proposta de extensão aqui se concentra nesta ideia. Uma maneira de estimar os coeficientes $p_{j}[n, p]$ ao longo do espaço é minimizar o erro de predição do algoritmo LIFTING SCHEME ao longo do tempo:

$$
p_{j}^{*}[n, p] \in \underset{p_{j}[n, p]}{\operatorname{argmin}} \sum_{t}\left(a_{t, j-1}[n]-\sum_{p \in V_{j}} p_{j}[n, p] a_{t, j-1}[p]\right)^{2},
$$

em que $a_{t, 0}[p]=z_{t}[p]$. Da mesma maneira, $u_{j}[n, p]$ pode ser estimado buscando minimizar o erro de atualização do algoritmo LIFTING SCHEME ao longo do tempo:

$$
u_{j}^{*}[n, p] \in \underset{u_{j}[n, p]}{\operatorname{argmin}} \sum_{t}\left(a_{t, j-1}[p]+\sum_{n \in C_{j}} u_{j}[n, p] d_{t, j}[n]\right)^{2} .
$$

Tais estimadores lineares podem ser obtidos pelo método de mínimos quadrados. Estimadores não lineares também podem ser construídos utilizando a mesma ideia. Isso permitiria obter representações esparsas altamente genéricas. A princípio, as equações (5.1) e (5.2) podem ainda ser generalizadas para estimar os coeficientes $p_{j}[n, p]$ e $u_{j}[n, p]$ com base em um modelo de regressão envolvendo uma covariável $x_{t}$ :

$$
a_{t, j-1}[n] \sim \sum_{p \in V_{j}} p_{j}[n, p] x_{t} a_{t, j-1}[p]
$$

$\mathrm{e}$

$$
a_{t, j-1}[p] \sim-\sum_{n \in C_{j}} u_{j}[n, p] x_{t} d_{t, j}[n]
$$

Um possível objeto de estudo seria a estabilidade e inversão desses tipos de transformações.

Um outro benefício da descrição acima seria obter transformações que não dependem de 
penalização. Isso significa que todos os resultados já conhecidos da teoria geral de representação esparsa podem ser aplicados conjuntamente com o algoritmo proposto. Por exemplo, a otimização dos coeficientes $p_{j}[n, p]$ e $u_{j}[n, p]$ poderia ser feita de forma a garantir que os sinais reconstruídos pertencessem a uma classe de sinais definidos a priori, como funções de espalhamento.

Finalmente, nota-se que extensões do algoritmo LIFTING SCHEME permitirão avançar o estudo sinais de alta dimensão em geral, em especial, o estudo do cérebro humano. Muitos métodos tradicionais de processamento de sinais em neurociência utilizam filtros estacionários que limitam as análises em grande parte. Essa abordagem vai certamente render frutos nessa área de pesquisa. O domínio total de tais transformações permitiria estudar a relação entre representações esparsas e o algoritmo ICA em FMRI, com implicações importantes para o estudo do estado de repouso. Nesta tese, foi demonstrado que ICA no estado de repouso de fato seleciona componentes esparsos. Além disso, mostrou-se dependência dinâmica entre componentes que são considerados independentes pela literatura. Outra aplicação importante em FMRI seria o desenvolvimento de filtros não estacionários para remoção de ruído em imagens de alta resolução, evitando o uso de filtros estacionários que deterioram a resolução espacial das imagens. 


\section{Apêndice A}

\section{ICA e SCA}

Neste capítulo, conceitos básicos que auxiliam a compressão dos demais capítulos desta tese são apresentados. Na primeira seção, duas técnicas de separação cega de canais - análise de componentes independentes e análise de componentes esparsos - são abordadas.

\section{A.1 Separação Cega de Fontes}

Separação cega de fontes refere-se a técnicas de processamento de sinais que buscam reconstruir um sinal a partir de observações ruidosas nas quais o sinal de interesse aparece misturado a outros sinais [CJ10]. Um exemplo clássico desse problema é conhecido como the cocktail party problem onde conversações de $M$ pessoas são gravadas simultaneamente utilizando $N$ microfones espalhados em diferentes lugares. O objetivo é reconstruir a conversa de cada participante utilizando somente as misturas das conversas originais gravadas pelos diferentes microfones.

Separação cega de fontes possui uma enorme gama de aplicações em diferentes áreas, incluindo telecomunicações [TJ95], biomedicina $\left[\mathrm{JMH}^{+} 00\right]$ e sensoriamento remoto $\left[\mathrm{MHS}^{+} 08\right.$ ]. Em sistemas multivariados de comunicação, o objetivo é reconstruir a informação transmitida por múltiplos canais que chegam às antenas de recepção misturados. Em eletroencefalografia, o objetivo é reconstruir os sinais de diferentes dipolos espalhados pelo cérebro utilizando múltiplos sensores localizados no escalpo. A reconstrução desses sinais permite analisar a circuitaria cerebral em função do processamento cognitivo. Em sensoriamento re- 
moto, múltiplos sensores localizados em satélites captam informações espectrais de múltiplas substâncias presentes no solo. Novamente, os espectros observados são misturas de vários espectros. O objetivo é detectar a presença de determinados padrões espectrais que fazem parte da mistura e, consequentemente, detectar a existência de determinadas substâncias.

A maneira mais simples de expressar os sinais em problemas de separação cega de fontes é através de um modelo linear da seguinte forma:

$$
\mathbf{Z}=\mathbf{A X}+\mathbf{V}
$$

em que $\mathbf{Z} \in \mathbb{R}^{N \times T}$ é uma matriz cujas colunas representam os sinais observados, $\mathbf{X} \in \mathbb{R}^{M \times T}$ é uma matriz cujas linhas representam as fontes, $\mathbf{A} \in \mathbb{R}^{N \times M}$ é uma matriz que modela a mistura das fontes e $\mathbf{V} \in \mathbb{R}^{N \times T}$ é uma matriz de ruído gaussiano. As constantes $T, N$ e $M$ denotam respectivamente número de observações, número de sinais e número de fontes.

\section{A.1.1 Análise de Componentes Independentes}

ICA é um método clássico para reconstruir $\mathbf{X}$ utilizando $\mathbf{Z}$ sobre a hipótese de que as linhas de $\mathbf{X}$ são independentes e não gaussianas. Sabe-se que ICA obtém bons resultados quando as hipóteses acima são verdadeiras [HKO04].

Inicialmente, considere que $\mathbf{V}=\mathbf{0}$, portanto, tem-se que $\mathbf{Z}=\mathbf{A X}$. ICA utiliza momentos de ordem superior para maximizar a independência entre as fontes identificadas. Isso distingue ICA de métodos que utilizam somente a informação contida na matriz de covariância de Z, por exemplo, a análise fatorial [HS07]. Assim, para simplificar os cálculos envolvendo momentos de ordem superior, $\mathbf{Z}$ é centralizado e descorrelacionado temporalmente por um

operador linear, i.e., $\tilde{\mathbf{Z}}=\mathbf{B Z}=\mathbf{B A X}$ tal que $E\{\tilde{\mathbf{Z}}\}=\mathbf{0}$ e $E\left\{\tilde{\mathbf{Z}} \tilde{\mathbf{Z}}^{\mathrm{T}}\right\}=\mathbf{I}$. Isso significa que o operador linear BA representa uma rotação das colunas de $\mathbf{X}$. O objetivo é encontrar uma matriz ortogonal $\mathbf{C}$ que desfaça essa rotação.

Existem várias abordagens na literatura que utilizam ICA para separar fontes independentes [CJ10, LCG10]. O algoritmo de ICA mais popular busca, para cada fonte, uma combinação linear das linhas de $\tilde{\mathbf{Z}}$ que maximize uma medida de não gaussianidade baseado no princípio de que a combinação linear de variáveis independentes possui uma dis- 
tribuição mais gaussiana que qualquer uma das variáveis isoladamente. Portanto, o sinal $u=\sum_{n=1}^{N} c_{n} z_{n, t}=\mathbf{c}^{\mathrm{T}} \mathbf{z}$, para todo $t$, se torna menos gaussiano a medida que ele se aproxima de um dos componentes independentes originais. Esse é o princípio do algoritmo de ICA conhecido como fastICA [Hyv99] cuja medida de não gaussianidade é dada por uma aproximação da entropia não negativa de $u$ :

$$
J(u) \approx[E\{G(u)\}-E\{\nu\}]^{2}=\left[E\left\{G\left(\mathbf{c}^{\mathrm{T}} \mathbf{z}\right)\right\}-E\{\nu\}\right]^{2},
$$

em que $G$ representa qualquer função não quadrática de $u$ e $\nu$ é uma variável gaussiana padronizada. Para $G(u)=u^{4}$, tem-se uma aproximação baseada na curtose de $u$.

Para minimizar (A.2) em função de c, utiliza-se um método de otimização de ponto fixo que fornece a seguinte equação de atualização:

$$
\begin{array}{r}
\mathbf{c} \leftarrow E\left\{\mathbf{z} g\left(\mathbf{c}^{\mathrm{T}} \mathbf{z}\right)\right\}-E\left\{g^{\prime}\left(\mathbf{c}^{\mathrm{T}} \mathbf{z}\right)\right\} \mathbf{c} \\
\mathbf{c} \leftarrow \mathbf{c} /\|\mathbf{c}\|^{2},
\end{array}
$$

em que $g$ é a derivada de $G$ e $g^{\prime}$ é a derivada de $g$.

Interações baseadas somente nas equações (A.3) e (A.4) garantem identificação de uma fonte somente. Para estimar duas fontes ou mais utilizando o princípio de maximização da não gaussianidade, o algoritmo fastICA usa o fato que se duas fontes $\mathbf{c}_{i}$ e $\mathbf{c}_{j}$ são independentes, elas são necessariamente ortogonais. Então, para estimar vários componentes independentes, fastICA garante que $\mathbf{C}=\left[\mathbf{c}_{1}, \ldots, \mathbf{c}_{M}\right]$ seja uma matriz ortogonal.

A ortogonalização pode ser obtida pelo método clássico de Gram-Schmidt. Entretanto, o método de Gram-Schmidt privilegia certas direções em detrimento de outras, podendo gerar um acúmulo de erros durante as interações [HKO04]. Uma alternativa é utilizar um método de ortogonalização simétrica, por exemplo, utilizando a seguinte equação:

$$
\mathbf{C} \leftarrow\left(\mathbf{C C}^{\mathrm{T}}\right)^{-1 / 2} \mathbf{C}
$$

O algoritmo fastICA para estimar $M$ fontes independentes é implementado de acordo com a tabela A.1. 
Tabela A.1: Algoritmo fastICA para fontes independentes

1. Dado $\tilde{\mathbf{Z}}$ centralizado e descorrelacionado.

2. Para $1 \leq i \leq M$, selecione valores para $\mathbf{c}_{i}$ arbitrariamente.

3. Ortogonalize $\mathbf{C}$ segundo a equação (A.5).

4. Para cada $i$, obtenha $\mathbf{c}_{i}$ segundo as equações (A.3) e (A.4).

5. Ortogonalize $\mathbf{C}$ segundo a equação (A.5).

6. Volte ao passo 4 até $\mathbf{c}_{i}$ convergir para todo $i$.

Finalmente, quando $\mathbf{V} \neq \mathbf{0}$, o algoritmo fastICA pode ser modificado tal que o viés devido ao ruído seja reduzido. A situação fica bastante simples quando a medida de não gaussianidade é a curtose, pois a curtose não é afetada por ruído gaussiano. Mesmo assim, a matriz de covariância de $\mathbf{V}$ precisa ser levada em consideração durante o pré-processamento de $\mathbf{Z}$. Seja $\Sigma$ a matriz de covariância de $\mathbf{V}$ e $\Sigma_{\mathbf{Z}}$ a matriz de covariância de $\mathbf{Z}$, o processo de centralização e descorrelação de $\mathbf{Z}$ deve ser realizado da seguinte maneira:

$$
\tilde{\mathbf{Z}}=\left(\Sigma_{\mathbf{Z}}-\Sigma\right)^{-1 / 2}\left(\mathbf{Z}-\mu_{\mathbf{Z}}\right)
$$

em que $\mu_{\mathbf{Z}}$ denota a média temporal de $\mathbf{Z}$.

No fastICA com ruído, as equações (A.3) e (A.4) também são modificadas, obtendo um método mais consistente na presença de ruído:

$$
\begin{array}{r}
\mathbf{c}^{*} \leftarrow E\left\{\tilde{\mathbf{z}} g\left(\mathbf{c}^{\mathrm{T}} \tilde{\mathbf{z}}\right)\right\}-(\operatorname{Id}-\tilde{\Sigma}) E\left\{g^{\prime}\left(\mathbf{c}^{\mathrm{T}} \tilde{\mathbf{z}}\right)\right\} \mathbf{c} \\
\mathbf{c}^{*} \leftarrow \mathbf{c}^{*} /\left\|\mathbf{c}^{*}\right\|^{2},
\end{array}
$$

em que $\tilde{\Sigma}=\left(\Sigma_{\mathbf{Z}}-\Sigma\right)^{1 / 2} \Sigma\left(\Sigma_{\mathbf{Z}}-\Sigma\right)^{1 / 2}$

\section{A.1.2 Análise de Componentes Esparsos}

Diferentemente do ICA, a ideia por trás do SCA é geométrica [BZ01, ZP $\left.{ }^{+} 01\right]$. Isso pode ser ilustrado através de uma modificação simples do problema de reconstrução em the cocktail party problem que será denominado aqui por the posh talk. Suponha que, durante a conversa, cada participante decida educadamente falar somente caso nenhum de seus parceiros estejam 
falando. Isso significa que o conjunto de pontos $\{1, \ldots, T\}$ pode ser particionado em $M$ subconjuntos disjuntos $\tau_{m}$, um para cada participante. Consequentemente, de acordo com o modelo (A.1), cada coluna da matriz $\mathbf{Z}$ fica preponderantemente caracterizada por uma única fonte, i.e.

$$
\mathbf{z}_{t}=x_{m, t} \mathbf{a}_{m}+\mathbf{v}_{t}
$$

em que $\mathbf{z}_{t}, \mathbf{a}_{m}$ e $\mathbf{v}_{t}$ são respectivamente colunas das matrizes $\mathbf{Z}, \mathbf{A}$ e $\mathbf{V}$.

A equação (A.9) indica que os conjuntos de vetores $\left\{\mathbf{z}_{t}\right\}_{t \in \tau_{m}}$, para $1 \leq m \leq M$, se localizam aproximadamente ao longo de linhas que passam pela origem e seguem nas direções $\mathbf{a}_{m}$, em outras palavras, o gráfico de dispersão de $\left\{\mathbf{z}_{t}\right\}_{t \in\{1, \ldots, T\}}$ pode ser agrupado em $M$ grupos, um para cada fonte. Portanto, as colunas da matriz A podem ser estimadas por um algoritmo que agrupe $\left\{\mathbf{z}_{t}\right\}_{t \in\{1, \ldots, T\}}$ ao longo de retas no espaço $N$-dimensional e calcule os parâmetros de inclinação de cada reta. Uma vez estimada a matriz de misturas, as fontes podem ser reconstruídas utilizando uma inversa generalizada de $\mathbf{A}$, por exemplo, a pseudoinversa de Moore-Penrose.

Considere o seguinte exemplo. Três fontes que seguem um modelo ARIMA,

$$
y_{t}^{m}=\sin (0.125 t)+0.5 y_{t-1}^{m}+0.5 w_{t-1}^{m}+w_{t-1}^{m},
$$

para $1 \leq m \leq 3$, em que $w_{t}^{m}$ segue uma distribuição normal padronizada, são amostradas de forma que somente uma delas está ativa a cada instante de tempo, ou seja:

$$
\begin{array}{r}
x_{t}^{m}=y_{t}^{m}, \text { se } \mathbf{U}_{t}=m, \\
x_{t}^{m}=0, \text { caso contrário, }
\end{array}
$$

em que $\mathbf{U}_{t}(1,3)$ representa uma distribuição uniforme discreta que pode assumir três valores $\{1,2,3\}$. Suponha também que as fontes são observadas somente por dois sensores de acordo e segundo a matriz de misturas abaixo:

$$
\mathbf{A}=\left[\begin{array}{ccc}
0.5 & -0.5 & 0 \\
0.5 & 0.5 & -0.5
\end{array}\right]
$$




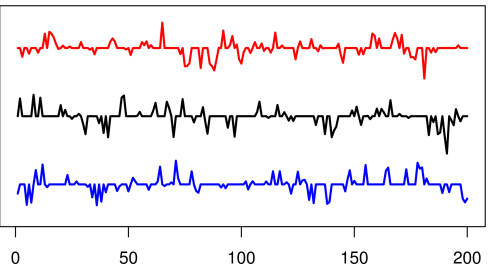

a

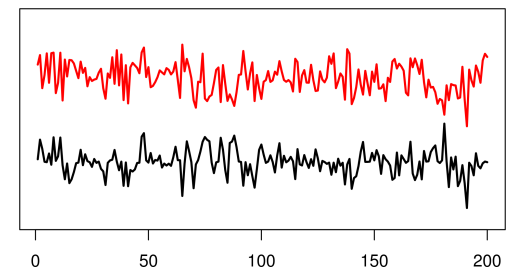

$\mathrm{b}$

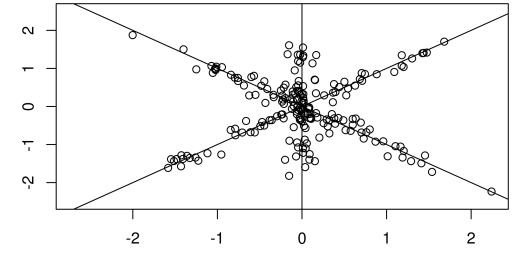

$\mathrm{C}$

Figura A.1: Fontes simuladas de acordo com a equação (A.11) (a). Sinais observados de acordo com o modelo (A.14) (b). Gráfico de dispersão dos sinais observados de acordo com o modelo (A.14) (c).

O modelo final é dado por

$$
z_{t}^{n}=\sum_{n=1}^{2} \sum_{m=1}^{3} a_{n, m} x_{t}^{m}+v_{t}^{n}
$$

para $1 \leq n \leq 2$, em que $v_{t}^{m}$ segue uma distribuição normal com média zero e desvio padrão igual a 0.1. A Figura A.1 mostra os gráficos de $x_{t}^{m}$ (a) e $z_{t}^{n}$ (b) livres de escala para fins ilustrativos. A Figura A.1 (c) mostra claramente o efeito das colunas da matriz A sobre o gráfico de dispersão das variáveis $z_{t}^{1}$ e $z_{t}^{2}$ e como essa informação poderia ser utilizada para estimá-las.

Embora a ideia acima seja simples, assumir que as fontes possuem um suporte temporal disjunto possui pouco efeito prático [CJ10]. Para superar essa limitação, representações esparsas entram em jogo com o objetivo de esparsificar os sinais, tornando seus suportes aproximadamente disjuntos em algum domínio transformado [GTCB07, GL06, LCA03, ZP ${ }^{+}$01]. Transformações esparsas de interesse incluem transformadas WAVELETS e seus derivados (análise de multiresolução, análise de tempo-frequência, pacotes WAVELETS, etc) [Don01, DE03]. Após uma transformação esparsa bem sucedida, a matriz de misturas A e as fontes $\mathbf{X}$ podem ser estimadas como descrito acima.

A Tabela A.2 apresenta uma estrutura genérica para algoritmos de SCA que consiste fundamentalmente em três etapas: construir uma representação esparsa dos dados, estimar a matriz de misturas e reconstruir as fontes. Se as fontes são suficientemente esparsas, a separação cega pode ser realizada diretamente no domínio original dos dados. Caso contrário, é necessário realizar uma transformação que os torne mais esparsos. Dado um dicionário de funções, geralmente, existem infinitas soluções para o problema de representação esparsa utilizando os elementos do dicionário. Entretanto, é possível obter unicidade em decomposições esparsas utilizando algoritmos de programação linear, por exemplo, o método de regulariza- 
Tabela A.2: Algoritmo SCA genérico para fontes esparsas

1. Dado $\tilde{\mathbf{Z}}$ e um dicionário de funções $\left\{\Phi_{i}\right\}_{i}$.

2. Construir uma representação esparsa de $\tilde{\mathbf{Z}}$ utilizando $\left\{\Phi_{i}\right\}_{i}$.

3. Estimar A pelo agrupamento de $\tilde{\mathbf{Z}}$ segundo suas características geométricas.

4. Reconstruir as fontes pela redução da dimensionalidade dos grupos obtidos no passo 3.

ção por grupos. Uma vez que os dados se tornam esparsos, a matriz A pode ser estimada por um algoritmo de agrupamento levando em conta características geométricas das fontes que são posteriormente reconstruídas por métodos de redução de dimensionalidade. 
64 APÊNDICE A 


\section{Apêndice B}

\section{Wavelets}

Este capítulo revisa os conceitos básicos da transformada WAVELETS.

\section{B.1 Tranformada WAVELETS}

WAVELETs é uma coleção de funções $\left\{\psi_{j, k}(t),(j, k) \in \mathbb{Z} \times \mathbb{Z}\right\}$ que constitui uma base ortogonal do espaço de funções $L^{2}$ e, portanto, permite que qualquer função $x(t) \in L^{2}$ tenha uma representação em termos de seus elementos, i.e.,

$$
x(t)=\sum_{k \in \mathbb{Z}} \sum_{j \in \mathbb{Z}} d_{j, k} \psi_{j, k}(t) .
$$

O mapeamento $W: L^{2} \rightarrow \mathbb{R}^{\mathbb{Z} \times \mathbb{Z}}$ dado por

$$
x(t) \rightarrow\left\{d_{j, k}\right\}_{(j, k) \in \mathbb{Z} \times \mathbb{Z},}
$$

é denominado a transformada WAVELETS de $x(t)\left[\mathrm{D}^{+} 92\right]$.

Em muitos problemas de processamento de sinais (compressão de dados, remoção de ruído, caracterização de sinais), WAVELETS são consideradas bases ótimas por aproximar uma grande variedade de classe de funções utilizando poucos coeficientes da base [Mal08]. Por definição, os coeficientes $d_{j, k}$ podem ser obtidos pelo produto interno entre $x(t)$ e $\psi_{j, k}(t)$

$$
d_{j, k}=\left\langle x(t), \psi_{j, k}(t)\right\rangle=\int_{\mathbb{R}} \psi_{j, k}(t) x(t) d t
$$


Entretanto, calcular o produto desta forma requer muito processamento. Felizmente, algoritmos mais rápidos e que demandam menos memória existem devido a estrutura peculiar imposta pela análise de multiresolução [Mal89].

O conjunto $\mathbf{W}_{j}=\left\{\psi_{j, k}(t), k \in \mathbb{Z}\right\}$ define um subespaço de $L^{2}$ que caracteriza os detalhes de $x(t)$. O nível de detalhe descrito por $\mathbf{W}_{j}$ cresce a medida em que $j \rightarrow-\infty$ e $\mathbf{W}_{j+1} \subset \mathbf{W}_{j}$. Tal decomposição é factível graças à análise de multiresolução [Mal89] em que uma cadeia de espaços de aproximação aninhados $\mathbf{V}_{1} \subset \mathbf{V}_{0} \subset \mathbf{V}_{-1} \ldots$ é construída de modo que se $x(t) \in \mathbf{V}_{j}$, então $x(2 t) \in \mathbf{V}_{j+1}$. O espaço $\mathbf{V}_{j}$ é gerado pela dilatação e translação de uma função $\phi(t)$, denominada função de escala, de forma que $\mathbf{V}_{j}$ é o espaço de funções geradas por combinações lineares de $\left\{\phi_{j . k}(t)=\sqrt{2} \phi\left(2^{-j} t-k\right), k \in \mathbb{Z}\right\}$. O espaço $\mathbf{W}_{j+1}$ é o complemento ortogonal de $\mathbf{V}_{j+1}$ em $\mathbf{V}_{j}$, isto é, $\mathbf{V}_{j}=\mathbf{V}_{j+1}+\mathbf{W}_{j+1}$. Pode ser demonstrado que $\mathbf{W}_{j}$ é gerado pela dilatação e translação de uma a função $\psi(t)$ chamada de função WAVELET. Informações mais detalhadas sobre como obter tais funções podem ser obtidas em [D+92, Vid99b, Mal08].

Suponha que $x(t) \in \mathbf{V}_{j}$

$$
x(t)=\sum_{k \in \mathbb{Z}} c_{j, k} \phi_{j, k}(t)
$$

Como $\mathbf{V}_{j}=\mathbf{V}_{j+1}+\mathbf{W}_{j+1}$, a equação (B.4) pode ser reescrita como

$$
x(t)=\sum_{k \in \mathbb{Z}} c_{j+1, k} \phi_{j+1, k}(t)+\sum_{k \in \mathbb{Z}} d_{j+1, k} \psi_{j+1, k}(t) .
$$

Tem-se também que

$$
\frac{1}{2} \phi\left(\frac{t}{2}\right)=\sum_{k \in \mathbb{Z}} h_{k} \phi(t-k)
$$

e

$$
\frac{1}{2} \psi\left(\frac{t}{2}\right)=\sum_{k \in \mathbb{Z}} g_{k} \phi(t-k),
$$

pois $\mathbf{V}_{1} \subset \mathbf{V}_{0}$ e $\mathbf{W}_{1} \subset \mathbf{V}_{0}$.

As equações (B.5), (B.6) e (B.7) permitem obter os coeficientes $c_{j+1, k}$ e $d_{j+1, k}$ de maneira recursiva através do produto interno de (B.5) em relação a $\phi_{j+1, k}(t)$ e $\psi_{j+1, k}(t)$, respectiva- 
mente

$$
\begin{array}{r}
c_{j+1, k}=\left\langle x(t), \phi_{j+1, k}(t)\right\rangle=\sum_{n \in \mathbb{Z}} c_{j, n}\left\langle\phi_{j, n}(t), \phi_{j+1, n}(t)\right\rangle \\
=\sum_{n \in \mathbb{Z}} c_{j, n} \sum_{m \in \mathbb{Z}} h_{m}\left\langle\phi_{j, n}(t-n), \phi_{j, n}(t-m-2 k)\right\rangle=\sum_{n \in \mathbb{Z}} c_{j, n} h_{n-2 k}
\end{array}
$$

e

$$
\begin{array}{r}
d_{j+1, k}=\left\langle x(t), \psi_{j+1, k}(t)\right\rangle=\sum_{n \in \mathbb{Z}} c_{j, n}\left\langle\phi_{j, n}(t), \psi_{j+1, n}(t)\right\rangle \\
=\sum_{n \in \mathbb{Z}} c_{j, n} \sum_{m \in \mathbb{Z}} g_{m}\left\langle\phi_{j, n}(t-n), \phi_{j, n}(t-m-2 k)\right\rangle=\sum_{n \in \mathbb{Z}} c_{j, n} g_{n-2 k} .
\end{array}
$$

Assim, os coeficientes de aproximação no nível $j+1$ são obtidos pela convolução dos coeficientes de aproximação no nível $j$ com o filtro $h_{k}$ invertido no tempo e seguido por uma operação de subamostragem por um fator de dois. Os coeficientes de detalhe no nível $j+1$ são obtidos pela convolução dos coeficientes de aproximação no nível $j$ com o filtro $g_{k}$ invertido no tempo e seguido por uma operação de subamostragem por um factor de dois.

Na prática, considera-se que $c_{0, k} \equiv x(k \Delta t)$, ou seja, $c_{0, k}$ é o resultado de um processo de amostragem de $x(t)$, em que $1 / \Delta t$ é a frequência de amostragem. As equações (B.8) e (B.9) indicam que $c_{0, k}$ é decomposto em componentes de baixa e alta frequência pela sua convolução com os filtros passa baixo $g_{k}$ e passa alto $h_{k}$, respectivamente, antes da operação de subamostragem. Esse processo de decomposição continua iterando sobre os componentes de baixa frequência enquanto os coeficientes de alta frequência são mantidos intactos. Na última iteração, tanto os coeficientes de baixa frequência quanto os de alta frequência são mantidos. 


\section{B.1.1 A Transformada WAVELET de Haar}

A função WAVELET da transformada WAVELET de Haar possui a seguinte forma:

$$
\psi(t)= \begin{cases}1 & \text { se } 0 \leq t \leq 1 / 2 \\ -1 & \text { se } 1 / 2 \leq t \leq 1 \\ 0 & \text { caso contrário. }\end{cases}
$$

Embora a transformada WAVELET de Haar constitui o primeiro exemplo de transformada WAVELETS aplicada ao longo da história, WAVELETS de Haar e, consequentemente, seus filtros $h_{k}$ e $g_{k}$ possuem suporte compacto. De fato, elas possuem o menor suporte dentre todas funções WAVELETS existentes. Isso permite obter representações bem adaptadas para sinais diversos pontos de singularidade utilizando algoritmos com o mínimo de processamento possível.

\section{B.1.2 A Transformada WAVELET Multivariada}

As equações anteriores podem ser estendidas para qualquer dimensão pelo produto tensorial das funções de escala e WAVELET. No caso tridimensional, os coeficientes de aproximação são obtidos pela seguinte função de escala $\phi(x, y, z)=\phi(x) \phi(y) \phi(z)$ e os coeficientes de detalhe são obtidos pelas funções WAVELETS direcionais $\psi_{1}(x, y, z)=\phi(x) \phi(y) \psi(z), \psi_{2}(x, y, z)=$ $\phi(x) \psi(y) \phi(z), \psi_{3}(x, y, z)=\phi(x) \psi(y) \psi(z), \psi_{4}(x, y, z)=\psi(x) \phi(y) \phi(z), \psi_{5}(x, y, z)=\psi(x) \phi(y) \psi(z)$, $\psi_{6}(x, y, z)=\psi(x) \psi(y) \phi(z)$ e $\psi_{7}(x, y, z)=\psi(x) \psi(y) \psi(z)$.

\section{B.1.3 Pacotes WaVElets}

A transformada WAVELETS convencional decompõe os coeficientes de aproximação em componentes de baixa e alta frequência. Na transformada por pacotes WAVELETS a decomposição em componentes de baixa e alta frequência é feita tanto nos coeficientes de detalhe quanto nos coeficientes de aproximação. Consequentemente, o conjunto de todos os coeficientes gerados nesse processo é bastante redundante. De fato, a transformada por pacotes WAVELETS representa uma biblioteca de funções ou um FRAME que pode ser gerado pela 
dilatação e translação de funções obtidas pela seguinte recursão

$$
W_{2 n}(t)=\sqrt{2} \sum_{k \in \mathbb{Z}} h_{k} W_{n}(2 t-k),
$$

$\mathrm{e}$

$$
W_{2 n+1}(t)=\sqrt{2} \sum_{k \in \mathbb{Z}} g_{k} W_{n}(2 t-k) .
$$

em que $W_{0}(t)=\phi$ e $W_{1}(t)=\psi$ [CW92]. 
70 APÊNDICE B 


\section{Apêndice C}

\section{O Algoritmo EM}

Considere novamente o modelo linear $(1.9,1.10)$ cuja representação por espaço de estados é dada pelo seguinte conjunto de equações

$$
\begin{aligned}
& \mathbf{x}_{t}=\mathbf{H} \mathbf{x}_{t-1}+\mathbf{w}_{t} \\
& \mathbf{z}_{t}=\mathbf{A} \mathbf{x}_{t}+\mathbf{v}_{t}
\end{aligned}
$$

em que $\mathbf{z}_{t}=\left[z_{1, t}, \cdots, z_{M, t}\right]^{\mathrm{T}}$ para $t \in\{1,2, \ldots, N\}$ são observações corrompidas por ruído iid com distribuição gaussiana $\mathbf{v}_{t} \sim \mathcal{N}(\mathbf{0}, \mathbf{R})$ e $\mathbf{x}_{t}=\left[x_{1, t}, \cdots, x_{K, t}\right]^{\mathrm{T}}$ é um vetor de estados que segue um modelo autoregressivo de primeira ordem com matriz de transição $\mathbf{H}$ e impulsionados por um processo de inovações também gaussiano $\mathbf{w}_{t} \sim \mathcal{N}(\mathbf{0}, \mathbf{Q})$.

Usando o fato que o processo acima é Markoviano e supondo que o estado inicial também seja Gaussiano $\mathbf{x}_{1} \sim \mathcal{N}\left(\boldsymbol{\mu}_{1}, \boldsymbol{\Sigma}_{1}\right)$, a solução padrão de (C.1,C.2) usa o algoritmo EM para maximizar iterativamente sua função de log-verossimilhança [SS82]

$$
\begin{gathered}
\log L(\boldsymbol{\theta}, \mathbf{x} \mid \mathbf{z}) \quad-\frac{1}{2} \log \left|\boldsymbol{\Sigma}_{1}\right|-\frac{1}{2}\left(\mathbf{x}_{1}-\boldsymbol{\mu}_{1}\right)^{\mathrm{T}} \boldsymbol{\Sigma}_{1}^{-1}\left(\mathbf{x}_{1}-\boldsymbol{\mu}_{1}\right) \\
-\frac{N-1}{2} \log |\mathbf{Q}|-\frac{1}{2} \sum_{t=2}^{N}\left(\mathbf{x}_{t}-\mathbf{H} \mathbf{x}_{t-1}\right)^{\mathrm{T}} \mathbf{Q}^{-1}\left(\mathbf{x}_{t}-\mathbf{H} \mathbf{x}_{t-1}\right) \\
-\frac{N}{2} \log |\mathbf{R}|-\frac{1}{2} \sum_{t=1}^{N}\left(\mathbf{z}_{t}-\mathbf{A} \mathbf{x}_{t}\right)^{\mathrm{T}} \mathbf{R}^{-1}\left(\mathbf{z}_{t}-\mathbf{A} \mathbf{x}_{t}\right),
\end{gathered}
$$

em que $\boldsymbol{\theta}$ representa os parâmetros $\boldsymbol{\mu}_{0}, \boldsymbol{\Sigma}_{0}, \mathbf{A}, \mathbf{R}, \mathbf{H}$ e $\mathbf{Q}, \mathbf{z}=\operatorname{vec}\left(\left[\mathbf{z}_{1} \cdots \mathbf{z}_{N}\right]\right), \mathbf{x}=\operatorname{vec}\left(\left[\mathbf{x}_{1} \cdots \mathbf{x}_{N}\right]\right)$ e vec representa o operador de empilhamento. 
A essência do algoritmo EM é obter a esperança condicional da verossimilhança (C.3) dados os dados e as estimativas dos parâmetros obtidos na $(i-1)$-ésima iteração do M-passo

$$
Q\left(\boldsymbol{\theta}, \hat{\boldsymbol{\theta}}^{(i)}\right)=\mathrm{E}\left[\log L(\boldsymbol{\theta}, \mathbf{x} \mid \mathbf{z}) \mid \mathbf{z}, \hat{\boldsymbol{\theta}}^{(i)}\right]
$$

sendo que (C.4) é a i-ésima iteração do E-passo. A i-ésima iteração do M-passo atualiza os parâmetros maximizando (C.4) em relação a $\boldsymbol{\theta}$. Mais precisamente, o algoritmo EM produz uma sequência de estimativas de estados e parâmetros alternando entre as seguintes etapas (até que um determinado critério de convergência seja satisfeito):

\section{E-passo}

No caso Gaussiano, o E-passo consiste em substituir os estados desconhecidos pelo seu valor esperado dado os dados [SS82]. No contexto de modelos de espaço de estados muitos pesquisadores fizeram amplo uso do suavizador de Rauch-Tung-Striebel (RTS) que é também, sobre a hipótese de gaussianidade, o estimador de máxima verossimilhança dos estados do sistema [RST65].

Denote por $\tilde{\mathbf{x}}_{t \mid s}$ a esperança condicional de $\mathbf{x}_{t}$ dado $\left\{\mathbf{z}_{1}, \mathbf{z}_{2}, \ldots, \mathbf{z}_{s}\right\}$, Considere as seguintes matrizes de covariância

$$
\mathbf{P}_{t \mid s}=\operatorname{cov}\left(\tilde{\mathbf{x}}_{t \mid s}\right)
$$

e

$$
\mathbf{P}_{t, t-1 \mid s}=\operatorname{cov}\left(\tilde{\mathbf{x}}_{t \mid s}, \tilde{\mathbf{x}}_{t-1 \mid s}\right)
$$

Dado A, R, H, Q, $\boldsymbol{\mu}_{1}$ e $\boldsymbol{\Sigma}_{1}$, defina $\tilde{\mathbf{x}}_{1 \mid 0}=\boldsymbol{\mu}_{1}$ e $\mathbf{P}_{1 \mid 0}=\boldsymbol{\Sigma}_{1}$. O suavizador de RTS consiste em três passos:

- Previsão:

$$
\begin{aligned}
\tilde{\mathbf{x}}_{t \mid t-1} & =\quad \mathbf{H} \tilde{\mathbf{x}}_{t-1 \mid t-1} \\
\mathbf{P}_{t \mid t-1} & =\mathbf{H P}_{t-1 \mid t-1} \mathbf{H}^{\mathrm{T}}+\mathbf{Q} .
\end{aligned}
$$




\section{- Filtragem:}

$$
\begin{aligned}
& \mathbf{K}_{t}=\mathbf{P}_{t \mid t-1} \mathbf{A}^{\mathrm{T}}\left(\mathbf{A P}_{t \mid t-1} \mathbf{A}^{\mathrm{T}}+\mathbf{R}\right)^{-1} \\
& \tilde{\mathbf{x}}_{t \mid t}=\quad \tilde{\mathbf{x}}_{t \mid t-1}+\mathbf{K}_{t}\left(\mathbf{z}_{t}-\mathbf{A} \tilde{\mathbf{x}}_{t \mid t-1}\right) \\
& \mathbf{P}_{t \mid t}=\quad \mathbf{P}_{t \mid t-1}-\mathbf{K}_{t} \mathbf{A} \mathbf{P}_{t \mid t-1},
\end{aligned}
$$

em que $\mathbf{K}_{t}$ é o ganho de Kalman.

\section{- Suavização:}

$$
\begin{array}{cc}
\mathbf{M}_{t}= & \mathbf{P}_{t \mid t} \mathbf{H}^{\mathrm{T}} \mathbf{P}_{t+1 \mid t}^{-1} \\
\tilde{\mathbf{x}}_{t \mid N}= & \tilde{\mathbf{x}}_{t \mid t}+\mathbf{M}_{t}\left(\tilde{\mathbf{x}}_{t+1 \mid N}-\mathbf{H} \tilde{\mathbf{x}}_{t \mid t}\right) \\
\mathbf{P}_{t \mid N}= & \mathbf{P}_{t \mid t}+\mathbf{M}_{t}\left(\mathbf{P}_{t+1 \mid N}-\mathbf{P}_{t+1 \mid t}\right) \mathbf{M}_{t}^{\mathrm{T}} .
\end{array}
$$

Nota 1. O cálculo do ganho de Kalman em (C.8) requer a inversão de uma matriz $M \times M$. Quando $K<<M$ e $\mathbf{R}^{-1}$ é diagonal, o ganho de Kalman pode ser posto em uma forma mais conveniente, usando uma igualdade matricial bem conhecida [Jaz70]

$$
\mathbf{P}_{t \mid t-1} \mathbf{A}^{\mathrm{T}}\left(\mathbf{A} \mathbf{P}_{t \mid t-1} \mathbf{A}^{\mathrm{T}}+\mathbf{R}\right)^{-1}=\left(\mathbf{I}+\mathbf{P}_{t \mid t-1} \mathbf{A}^{\mathrm{T}} \mathbf{R}^{-1} \mathbf{A}\right)^{-1} \mathbf{P}_{t \mid t-1} \mathbf{A}^{\mathrm{T}} \mathbf{R}^{-1}
$$

que requer somente a inversão de uma matriz $K \times K$.

Nota 2. O M-passo a seguir utiliza a matriz de covariância calculada pelas seguintes recursões

$$
\mathbf{P}_{t-1, t-2 \mid N}=\mathbf{P}_{t-1 \mid t-1} \mathbf{M}_{t-2}^{\mathrm{T}}+\mathbf{M}_{t-1}\left(\mathbf{P}_{t, t-1 \mid N}-\mathbf{H P}_{t-1 \mid t-1}\right) \mathbf{M}_{t-2}^{\mathrm{T}},
$$

para $t=N, N-1, \ldots, 2 \mathrm{e}$

$$
\mathbf{P}_{N, N-1 \mid N}=\left(\mathbf{I}_{K \times K}-\mathbf{K}_{N} \mathbf{A}\right) \mathbf{H} \mathbf{P}_{N-1 \mid N-1} .
$$

\section{M-passo}

Dado $\tilde{\mathbf{x}}_{t \mid N}, \mathbf{V}_{t \mid N}=\mathbf{P}_{t \mid N}+\tilde{\mathbf{x}}_{t \mid N} \tilde{\mathbf{x}}_{t \mid N}^{\mathrm{T}}$ e $\mathbf{V}_{t, t-1 \mid N}=\mathbf{P}_{t, t-1 \mid N}+\tilde{\mathbf{x}}_{t \mid N} \tilde{\mathbf{x}}_{t-1 \mid N}^{\mathrm{T}}$ obtidos na $i$-ésima iteração do E-passo e $\mathbf{z}_{t}$, os seguintes estimadores de $\mathbf{A}, \mathbf{H}, \mathbf{Q}, \boldsymbol{\mu}_{1}$ e $\boldsymbol{\Sigma}_{1}$ são obtidas pela 
maximização de $Q\left(\boldsymbol{\theta}, \hat{\boldsymbol{\theta}}^{(i)}\right)$.

$$
\begin{gathered}
\mathbf{A}=\left(\sum_{t=1}^{N} \mathbf{z}_{t} \tilde{\mathbf{x}}_{t \mid N}^{\mathrm{T}}\right)\left(\sum_{t=1}^{N} \mathbf{V}_{t \mid N}\right)^{-1}, \\
\mathbf{H}=\left(\sum_{t=2}^{N} \mathbf{V}_{t, t-1 \mid N}\right)\left(\sum_{t=2}^{N} \mathbf{V}_{t-1 \mid N}\right)^{-1}, \\
\mathbf{Q}=(N-1)^{-1}\left(\sum_{t=2}^{N} \mathbf{V}_{t \mid N}-\mathbf{H} \sum_{t=2}^{N} \mathbf{V}_{t, t-1 \mid N}\right), \\
\boldsymbol{\mu}_{1}=\tilde{\mathbf{x}}_{1 \mid N},
\end{gathered}
$$

e

$$
\boldsymbol{\Sigma}_{1}=\mathbf{V}_{1 \mid N}-\tilde{\mathbf{x}}_{1 \mid N} \tilde{\mathbf{x}}_{1 \mid N}^{\mathrm{T}},
$$




\section{Referências Bibliográficas}

[BAHS08] Randy L Buckner, Jessica R Andrews-Hanna e Daniel L Schacter. The brain's default network. Annals of the New York Academy of Sciences, 1124(1):1-38, 2008. 9

[Ban12] Peter A Bandettini. Functional mri: a confluence of fortunate circumstances. NeuroImage, 61(2):A3-A11, 2012. 1, 4

[BDBTS13] LA Baccalá, CSN De Brito, DY Takahashi e K Sameshima. Unified asymptotic theory for all partial directed coherence forms. Philosophical Transactions of the Royal Society of London A: Mathematical, Physical and Engineering Sciences, 371(1997):20120158, 2013. 31

[BDDS05] Christian F Beckmann, Marilena DeLuca, Joseph T Devlin e Stephen M Smith. Investigations into resting-state connectivity using independent component analysis. Philosophical Transactions of the Royal Society of London B: Biological Sciences, 360(1457):1001-1013, 2005. 3, 6, 8, 9, 15, 49

[BJBR09] Matthias Beckmann, Heidi Johansen-Berg e Matthew FS Rushworth. Connectivity-based parcellation of human cingulate cortex and its relation to functional specialization. The Journal of neuroscience, 29(4):1175-1190, 2009. 3

$\left[\mathrm{BJM}^{+} 12\right]$ Francis Bach, Rodolphe Jenatton, Julien Mairal, Guillaume Obozinski et al. Structured sparsity through convex optimization. Statistical Science, 27(4):450-468, 2012. 18

[BMB08] Rasmus M Birn, Kevin Murphy e Peter A Bandettini. The effect of respiration variations on independent component analysis results of resting state functional connectivity. Human brain mapping, 29(7):740-750, 2008. 6

$\left[\mathrm{BMZ}^{+} 10\right]$ Bharat B Biswal, Maarten Mennes, Xi-Nian Zuo, Suril Gohel, Clare Kelly, Steve M Smith, Christian F Beckmann, Jonathan S Adelstein, Randy L Buckner, Stan Colcombe et al. Toward discovery science of human brain function. Proceedings of the National Academy of Sciences, 107(10):4734-4739, 2010. 1, $8,9,15,17,32,45,48$

[BS04] Christian F Beckmann e Stephen M Smith. Probabilistic independent component analysis for functional magnetic resonance imaging. Medical Imaging, IEEE Transactions on, 23(2):137-152, 2004. 49

[BS09] Ed Bullmore e Olaf Sporns. Complex brain networks: graph theoretical analysis of structural and functional systems. Nature Reviews Neuroscience, 10(3):186-198, 2009. 7 
[BU99] Bharat B Biswal e John L Ulmer. Blind source separation of multiple signal sources of fmri data sets using independent component analysis. Journal of computer assisted tomography, 23(2):265-271, 1999. 3, 8, 9, 15, 49

[BV07] Randy L Buckner e Justin L Vincent. Unrest at rest: default activity and spontaneous network correlations. Neuroimage, 37(4):1091-1096, 2007. 7

[BYHH95] Bharat Biswal, F Zerrin Yetkin, Victor M Haughton e James S Hyde. Functional connectivity in the motor cortex of resting human brain using echo-planar mri. Magnetic resonance in medicine, 34(4):537-541, 1995. 5, 7

[BZ01] Pau Bofill e Michael Zibulevsky. Underdetermined blind source separation using sparse representations. Signal processing, 81(11):2353-2362, 2001. 60

[CAC07] Nicolle Correa, Tülay Adalı e Vince D Calhoun. Performance of blind source separation algorithms for fmri analysis using a group ica method. Magnetic resonance imaging, 25(5):684-694, 2007. 15

[CG09] Catie Chang e Gary H Glover. Effects of model-based physiological noise correction on default mode network anti-correlations and correlations. Neuroimage, 47(4):1448-1459, 2009. 6

[CGS $\left.{ }^{+} 11\right]$ Gang Chen, Daniel R Glen, Ziad S Saad, J Paul Hamilton, Moriah E Thomason, Ian H Gotlib e Robert W Cox. Vector autoregression, structural equation modeling, and their synthesis in neuroimaging data analysis. Computers in biology and medicine, 41(12):1142-1155, 2011. 6

$\left[\mathrm{CHA}^{+} 00\right]$ Dietmar Cordes, Victor M Haughton, Konstantinos Arfanakis, Gary J Wendt, Patrick A Turski, Chad H Moritz, Michelle A Quigley e M Elizabeth Meyerand. Mapping functionally related regions of brain with functional connectivity mr imaging. American Journal of Neuroradiology, 21(9):1636-1644, 2000. 5

$\left[\mathrm{CHA}^{+}\right.$01] Dietmar Cordes, Victor M Haughton, Konstantinos Arfanakis, John D Carew, Patrick A Turski, Chad H Moritz, Michelle A Quigley e M Elizabeth Meyerand. Frequencies contributing to functional connectivity in the cerebral cortex in ?resting-state? data. American Journal of Neuroradiology, 22(7):1326-1333, 2001. 6

[CHHM09] R Cameron Craddock, Paul E Holtzheimer, Xiaoping P Hu e Helen S Mayberg. Disease state prediction from resting state functional connectivity. Magnetic resonance in Medicine, 62(6):1619-1628, 2009. 3, 6

[CJ10] Pierre Comon e Christian Jutten. Handbook of Blind Source Separation: Independent component analysis and applications. Academic press, 2010. 57, 58, 62

[CJBN98] Roger L Claypoole Jr, Richard G Baraniuk e Robert D Nowak. Adaptive wavelet transforms via lifting. Em Acoustics, Speech and Signal Processing, 1998. Proceedings of the 1998 IEEE International Conference on, volume 3, páginas 1513-1516. IEEE, 1998. 55

[Com94] Pierre Comon. Independent component analysis, a new concept? Signal Process, 36(10):287-314, 1994. 10 
[Cor09] Jorge Cortés. Distributed kriged kalman filter for spatial estimation. Automatic Control, IEEE Transactions on, 54(12):2816-2827, 2009. 13

[CP07] Patrick L Combettes e Jean-Christophe Pesquet. Proximal thresholding algorithm for minimization over orthonormal bases. SIAM Journal on Optimization, 18(4):1351-1376, 2007. 15

[CPA04] V Calhoun, G Pearlson e Tülay Adali. Independent component analysis applied to fmri data: a generative model for validating results. Journal of VLSI signal processing systems for signal, image and video technology, 37(2-3):281291, 2004. 8

$\left[\mathrm{CPP}^{+} 13\right]$ Vince D Calhoun, Vamsi K Potluru, Ronald Phlypo, Rogers F Silva, Barak A Pearlmutter, Arvind Caprihan, Sergey M Plis e Tülay Adalı. Independent component analysis for brain fmri does indeed select for maximal independence. PloS one, 8(8):e73309, 2013. 17

[CSB10] David M Cole, Stephen M Smith e Christian F Beckmann. Advances and pitfalls in the analysis and interpretation of resting-state fmri data. Frontiers in systems neuroscience, 4:16, 2010. 3

[CW92] Ronald R Coifman e M Victor Wickerhauser. Entropy-based algorithms for best basis selection. Information Theory, IEEE Transactions on, 38(2):713718, 1992. 25, 69

[CW02] Noel Cressie e Christopher K Wikle. Space-time kalman filter. Encyclopedia of environmetrics, 1:10-17, 2002. 11

[CW05] Patrick L Combettes e Valérie R Wajs. Signal recovery by proximal forwardbackward splitting. Multiscale Modeling ES Simulation, 4(4):1168-1200, 2005. 23

[D $\left.{ }^{+} 92\right]$ Ingrid Daubechies et al. Ten lectures on wavelets, volume 61. SIAM, 1992. 65,66

[DDDM03] Ingrid Daubechies, Michel Defrise e Christine De Mol. An iterative thresholding algorithm for linear inverse problems with a sparsity constraint. arXiv preprint math/0307152, 6:20-41, 2003. 23

[DE03] David L Donoho e Michael Elad. Optimally sparse representation in general (nonorthogonal) dictionaries via ?1 minimization. Proceedings of the National Academy of Sciences, 100(5):2197-2202, 2003. 62

[DG09] Jessica S Damoiseaux e Michael D Greicius. Greater than the sum of its parts: a review of studies combining structural connectivity and resting-state functional connectivity. Brain Structure and Function, 213(6):525-533, 2009. $2,3,6$

[DH12] Gopikrishna Deshpande e Xiaoping Hu. Investigating effective brain connectivity from fmri data: past findings and current issues with reference to granger causality analysis. Brain connectivity, 2(5):235-245, 2012. 15 
[DJKP95] David L Donoho, Iain M Johnstone, Gérard Kerkyacharian e Dominique Picard. Wavelet shrinkage: asymptopia? Journal of the Royal Statistical Society. Series B (Methodological), 1:301-369, 1995. 23

[DK07] Michael Dewar e Visakan Kadirkamanathan. A canonical space-time state space model: state and parameter estimation. Signal Processing, IEEE Transactions on, 55(10):4862-4870, 2007. 13

[DLBDS ${ }^{+}$06] M De Luca, CF Beckmann, N De Stefano, PM Matthews e Stephen M Smith. fmri resting state networks define distinct modes of long-distance interactions in the human brain. Neuroimage, 29(4):1359-1367, 2006. 6

[DLR77] Arthur P Dempster, Nan M Laird e Donald B Rubin. Maximum likelihood from incomplete data via the em algorithm. Journal of the royal statistical society. Series B (methodological), 1:1-38, 1977. 13, 15

[Don01] David Leigh Donoho. Sparse components of images and optimal atomic decompositions. Constructive Approximation, 17(3):353-382, 2001. 62

[DRB $\left.{ }^{+} 06\right]$ JS Damoiseaux, SARB Rombouts, F Barkhof, P Scheltens, CJ Stam, Stephen M Smith e CF Beckmann. Consistent resting-state networks across healthy subjects. Proceedings of the national academy of sciences, 103(37):13848-13853, 2006. 1, 6, 8, 9, 15, 16, 32

[DRT ${ }^{+}$09] I Daubechies, E Roussos, S Takerkart, M Benharrosh, C Golden, K D'ardenne, W Richter, JD Cohen e J Haxby. Independent component analysis for brain fmri does not select for independence. Proceedings of the National Academy of Sciences, 106(26):10415-10422, 2009. 17, 48

[DS98] Ingrid Daubechies e Wim Sweldens. Factoring wavelet transforms into lifting steps. Journal of Fourier analysis and applications, 4(3):247-269, 1998. 54

$\left[\mathrm{EFS}^{+}\right.$02] Fabrizio Esposito, Elia Formisano, Erich Seifritz, Rainer Goebel, Renato Morrone, Gioacchino Tedeschi e Francesco Di Salle. Spatial independent component analysis of functional mri time-series: To what extent do results depend on the algorithm used? Human brain mapping, 16(3):146-157, 2002. 8

$\left[\mathrm{FAD}^{+} 11\right]$ DR Freestone, P Aram, Michael Dewar, Kenneth Scerri, David B Grayden e Visakan Kadirkamanathan. A data-driven framework for neural field modeling. NeuroImage, 56(3):1043-1058, 2011. 13

$\left[\mathrm{FAH}^{+}\right.$03] Karl J Friston, JOHN Ashburner, J Heather et al. Statistical parametric mapping. Neuroscience Databases: A Practical Guide, 1:237, 2003. 5

[FFLF93] KJ Friston, CD Frith, PF Liddle e RSJ Frackowiak. Functional connectivity: the principal-component analysis of large (pet) data sets. Journal of cerebral blood flow and metabolism, 13:5-5, 1993. 49

[FG10] Michael D Fox e Michael Greicius. Clinical applications of resting state functional connectivity. Frontiers in systems neuroscience, 4:19, 2010. 1

[FGT12] Peter Filzmoser, Moritz Gschwandtner e Valentin Todorov. Review of sparse methods in regression and classification with application to chemometrics. Journal of Chemometrics, 26(3-4):42-51, 2012. 18 
$\left[\mathrm{FMP}^{+}\right.$02] Peter Fransson, Klaus-Dietmar Merboldt, Karl Magnus Petersson, Martin Ingvar e Jens Frahm. On the effects of spatial filtering?a comparative fmri study of episodic memory encoding at high and low resolution. NeuroImage, 16(4):977984, 2002. 3

[FMS $\left.{ }^{+} 10\right]$ David A Feinberg, Steen Moeller, Stephen M Smith, Edward Auerbach, Sudhir Ramanna, Matthias Gunther, Matt F Glasser, Karla L Miller, Kamil Ugurbil e Essa Yacoub. Multiplexed echo planar imaging for sub-second whole brain fmri and fast diffusion imaging. PloS one, 5(12):e15710, 2010. 31

[FR07] Michael D Fox e Marcus E Raichle. Spontaneous fluctuations in brain activity observed with functional magnetic resonance imaging. Nature Reviews Neuroscience, 8(9):700-711, 2007. 5

[Fra05] Peter Fransson. Spontaneous low-frequency bold signal fluctuations: An fmri investigation of the resting-state default mode of brain function hypothesis. Human brain mapping, 26(1):15-29, 2005. 9

[Fri05] Karl J Friston. Models of brain function in neuroimaging. Annu. Rev. Psychol., 56:57-87, 2005. 14

[Fri11] Karl J Friston. Functional and effective connectivity: a review. Brain connectivity, 1(1):13-36, 2011. 17

$\left[\mathrm{FSV}^{+}\right.$05] Michael D Fox, Abraham Z Snyder, Justin L Vincent, Maurizio Corbetta, David C Van Essen e Marcus E Raichle. The human brain is intrinsically organized into dynamic, anticorrelated functional networks. Proceedings of the National Academy of Sciences of the United States of America, 102(27):9673$9678,2005.5$

[FZB10] Alex Fornito, Andrew Zalesky e Edward T Bullmore. Network scaling effects in graph analytic studies of human resting-state fmri data. Frontiers in systems neuroscience, 4:22, 2010. 3

[GFM ${ }^{+}$07] Michael D Greicius, Benjamin H Flores, Vinod Menon, Gary H Glover, Hugh B Solvason, Heather Kenna, Allan L Reiss e Alan F Schatzberg. Resting-state functional connectivity in major depression: abnormally increased contributions from subgenual cingulate cortex and thalamus. Biological psychiatry, 62(5):429-437, 2007. 1

[GKRM03] Michael D Greicius, Ben Krasnow, Allan L Reiss e Vinod Menon. Functional connectivity in the resting brain: a network analysis of the default mode hypothesis. Proceedings of the National Academy of Sciences, 100(1):253-258, 2003. 6,9

[GL06] Rémi Gribonval e Sylvain Lesage. A survey of sparse component analysis for blind source separation: principles, perspectives, and new challenges. Em ESANN'06 proceedings-14th European Symposium on Artificial Neural Networks, páginas 323-330. d-side publi., 2006. 10, 16, 62

[GR70] Gene H Golub e Christian Reinsch. Singular value decomposition and least squares solutions. Numerische mathematik, 14(5):403-420, 1970. 29 
[Gre08] Michael Greicius. Resting-state functional connectivity in neuropsychiatric disorders. Current opinion in neurology, 21(4):424-430, 2008. 1, 6, 9

[GSMD09] Michael D Greicius, Kaustubh Supekar, Vinod Menon e Robert F Dougherty. Resting-state functional connectivity reflects structural connectivity in the default mode network. Cerebral cortex, 19(1):72-78, 2009. 1, 6

[GSRM04] Michael D Greicius, Gaurav Srivastava, Allan L Reiss e Vinod Menon. Defaultmode network activity distinguishes alzheimer's disease from healthy aging: evidence from functional mri. Proceedings of the National Academy of Sciences of the United States of America, 101(13):4637-4642, 2004. 9

[GSS $\left.{ }^{+} 06\right]$ Sam J Gilbert, Stephanie Spengler, Jon S Simons, J Douglas Steele, Stephen M Lawrie, Christopher D Frith e Paul W Burgess. Functional specialization within rostral prefrontal cortex (area 10): a meta-analysis. Journal of cognitive neuroscience, 18(6):932-948, 2006. 3

[GTCB07] Pando Georgiev, Fabian Theis, Andrzej Cichocki e Hovagim Bakardjian. Sparse component analysis: a new tool for data mining. Em Data mining in biomedicine, páginas 91-116. Springer, 2007. 3, 15, 16, 49, 62

[HKO04] Aapo Hyvärinen, Juha Karhunen e Erkki Oja. Independent component analysis, volume 46. John Wiley \& Sons, 2004. 58, 59

[Hor03] Barry Horwitz. The elusive concept of brain connectivity. Neuroimage, 19(2):466-470, 2003. 2

[HPPP05] Henk JAM Heijmans, Béatrice Pesquet-Popescu e Gemma Piella. Building nonredundant adaptive wavelets by update lifting. Applied and Computational Harmonic Analysis, 18(3):252-281, 2005. 54, 55

[HR02] David J Heeger e David Ress. What does fmri tell us about neuronal activity? Nature Reviews Neuroscience, 3(2):142-151, 2002. 4

[HR11] Barry Horwitz e James B Rowe. Functional biomarkers for neurodegenerative disorders based on the network paradigm. Progress in neurobiology, 95(4):505509, 2011. 6

[HR13] Laura E Hughes e James B Rowe. The impact of neurodegeneration on network connectivity: a study of change detection in frontotemporal dementia. Journal of cognitive neuroscience, 25(5):802-813, 2013. 6

[HS07] Wolfgang Härdle e Léopold Simar. Applied multivariate statistical analysis. Springer Science \& Business Media, 2007. 58

[HSM04] Scott A Huettel, Allen W Song e Gregory McCarthy. Functional magnetic resonance imaging, volume 1. Sinauer Associates Sunderland, 2004. 1, 3, 5

[Hyv99] Aapo Hyvärinen. Fast ica for noisy data using gaussian moments. Em Circuits and Systems, 1999. ISCAS'99. Proceedings of the 1999 IEEE International Symposium on, volume 5, páginas 57-61. IEEE, 1999. 17, 59

$\left[\mathrm{HZ}^{+} 10\right]$ Junzhou Huang, Tong Zhang et al. The benefit of group sparsity. The Annals of Statistics, 38(4):1978-2004, 2010. 18 
[Jaz70] Andrew H Jazwinski. Stochastic Processes and Filtering Theory. Academic Press, 1970. 73

$\left[\mathrm{JMH}^{+} 00\right]$ Tzyy-Ping Jung, Scott Makeig, Colin Humphries, Te-Won Lee, Martin J Mckeown, Vicente Iragui e Terrence J Sejnowski. Removing electroencephalographic artifacts by blind source separation. Psychophysiology, 37(02):163178, 2000. 57

[Joh67] Stephen C Johnson. Hierarchical clustering schemes. Psychometrika, 32(3):241-254, 1967. 26

[KBBPPP11] Mounir Kaaniche, Amel Benazza-Benyahia, Béatrice Pesquet-Popescu e J-C Pesquet. Non-separable lifting scheme with adaptive update step for still and stereo image coding. Signal Processing, 91(12):2767-2782, 2011. 55

[KBC ${ }^{+}$92] Kenneth K Kwong, John W Belliveau, David A Chesler, Inna E Goldberg, Robert M Weisskoff, Brigitte P Poncelet, David N Kennedy, Bernice E Hoppel, Mark S Cohen e Robert Turner. Dynamic magnetic resonance imaging of human brain activity during primary sensory stimulation. Proceedings of the National Academy of Sciences, 89(12):5675-5679, 1992. 5

[KPPBBP12] Mounir Kaaniche, Béatrice Pesquet-Popescu, Amel Benazza-Benyahia e JeanChristophe Pesquet. Adaptive lifting scheme with sparse criteria for image coding. EURASIP Journal on Advances in Signal Processing, 2012(1):1-22, 2012. 54,55

[KRDV ${ }^{+}$12] Thilo Kellermann, Christina Regenbogen, Maarten De Vos, Carolin Mößnang, Andreas Finkelmeyer e Ute Habel. Effective connectivity of the human cerebellum during visual attention. The Journal of Neuroscience, 32(33):1145311460, 2012. 50

[LCA03] Yuanqing Li, Andrzej Cichocki e Shun-Ichi Amari. Sparse component analysis for blind source separation with less sensors than sources. Em ICA2003, páginas 89-94. Citeseer, 2003. 16, 62

[LCG10] Dominic Langlois, Sylvain Chartier e Dominique Gosselin. An introduction to independent component analysis: Infomax and fastica algorithms. Tutorials in Quantitative Methods for Psychology, 6(1):31-38, 2010. 17, 58

$\left[\mathrm{LGN}^{+}\right.$09] Kaiming Li, Lei Guo, Jingxin Nie, Gang Li e Tianming Liu. Review of methods for functional brain connectivity detection using fmri. Computerized Medical Imaging and Graphics, 33(2):131-139, 2009. 2, 7

[LHWY11] Jong-Hwan Lee, Ryuichiro Hashimoto, Cynthia G Wible e Seung-Schik Yoo. Investigation of spectrally coherent resting-state networks using non-negative matrix factorization for functional mri data. International Journal of Imaging Systems and Technology, 21(2):211-222, 2011. 3, 15

[LMS98] MJ Lowe, BJ Mock e JA Sorenson. Functional connectivity in single and multislice echoplanar imaging using resting-state fluctuations. Neuroimage, $7(2): 119-132,1998.5$

[Log08] Nikos K Logothetis. What we can do and what we cannot do with fmri. Nature, 453(7197):869-878, 2008. 4 
[LSS13] Megan H Lee, Christopher D Smyser e Joshua S Shimony. Resting-state fmri: a review of methods and clinical applications. American Journal of Neuroradiology, 34(10):1866-1872, 2013. 1, 6

[LVU07] Gabriele Lohmann, Kirsten G Volz e Markus Ullsperger. Using non-negative matrix factorization for single-trial analysis of fmri data. Neuroimage, 37(4):1148-1160, 2007. 49

[LW04] Nikos K Logothetis e Brian A Wandell. Interpreting the bold signal. Annu. Rev. Physiol., 66:735-769, 2004. 5

[Mal89] Stephane G Mallat. A theory for multiresolution signal decomposition: the wavelet representation. Pattern Analysis and Machine Intelligence, IEEE Transactions on, 11(7):674-693, 1989. 66

[Mal08] Stephane Mallat. A wavelet tour of signal processing: the sparse way. Academic press, 2008. 23, 65, 66

[MBHZSZ12] Fatemeh Mokhtari, Shahab K Bakhtiari, Gholam Ali Hossein-Zadeh e Hamid Soltanian-Zadeh. Discriminating between brain rest and attention states using fmri connectivity graphs and subtree svm. Em SPIE Medical Imaging, páginas 83144C-83144C. International Society for Optics and Photonics, 2012. 3

$\left[\mathrm{MBL}^{+} 10\right]$ Daniel S Margulies, Joachim Böttger, Xiangyu Long, Yating Lv, Clare Kelly, Alexander Schäfer, Dirk Goldhahn, Alexander Abbushi, Michael P Milham, Gabriele Lohmann et al. Resting developments: a review of fmri postprocessing methodologies for spontaneous brain activity. Magnetic Resonance Materials in Physics, Biology and Medicine, 23(5-6):289-307, 2010. 3

[MBVP14] Katja Macher, Andreas Böhringer, Arno Villringer e Burkhard Pleger. Cerebellar-parietal connections underpin phonological storage. The Journal of Neuroscience, 34(14):5029-5037, 2014. 50

[Mey00] Carl D Meyer. Matrix analysis and applied linear algebra. Siam, 2000. 28

[MGB ${ }^{+}$09] Boris Mailhé, Rémi Gribonval, Frédéric Bimbot, Mathieu Lemay, Pierre Vandergheynst e Jean-Marc Vesin. Dictionary learning for the sparse modelling of atrial fibrillation in ecg signals. Em Acoustics, Speech and Signal Processing, 2009. ICASSP 2009. IEEE International Conference on, páginas 465-468. IEEE, 2009. 18

[MGRA98] Kanti V Mardia, Colin Goodall, Edwin J Redfern e Francisco J Alonso. The kriged kalman filter. Test, 7(2):217-282, 1998. 13

[MH92] Stephane Mallat e Wen Liang Hwang. Singularity detection and processing with wavelets. Information Theory, IEEE Transactions on, 38(2):617-643, 1992. 15

[MHB06] Paul M Matthews, Garry D Honey e Edward T Bullmore. Applications of fmri in translational medicine and clinical practice. Nature Reviews Neuroscience, 7(9):732-744, 2006. 4 
[MHS ${ }^{+}$08] Saïd Moussaoui, Hafrun Hauksdottir, Frédéric Schmidt, Christian Jutten, Jocelyn Chanussot, David Brie, Sylvain Douté e Jon Atli Benediktsson. On the decomposition of mars hyperspectral data by ica and bayesian positive source separation. Neurocomputing, 71(10):2194-2208, 2008. 57

[MR99] Luis C Maas e Perry F Renshaw. Post-registration spatial filtering to reduce noise in functional mri data sets. Magnetic resonance imaging, 17(9):13711382, 1999. 3

[MS $\left.{ }^{+} 98\right]$ Martin J McKeown, Terrence J Sejnowski et al. Independent component analysis of fmri data: examining the assumptions. Human brain mapping, 6(5-6):368-372, 1998. 8

[Mur85] Fionn Murtagh. A survey of algorithms for contiguity-constrained clustering and related problems. The computer journal, 28(1):82-88, 1985. 15, 16, 26

$\left[\mathrm{MYP}^{+} 09\right]$ Aviv Mezer, Yossi Yovel, Ofer Pasternak, Tali Gorfine e Yaniv Assaf. Cluster analysis of resting-state fmri time series. Neuroimage, 45(4):1117-1125, 2009. 3

[PF13] Hae-Jeong Park e Karl Friston. Structural and functional brain networks: from connections to cognition. Science, 342(6158):1238411, 2013. 14

[PMJ95] C Puntonet, A Mansour e C Jutten. A geometrical algorithm for blind separation of sources. Em Actes du XV Colloque GRETSI 95, Juan-Les-Pins, 1995. 10

[PPPHP06] Gemma Piella, Béatrice Pesquet-Popescu, Henk JAM Heijmans e Gregoire Pau. Combining seminorms in adaptive lifting schemes and applications to image analysis and compression. Journal of Mathematical Imaging and Vision, 25(2):203-226, 2006. 54, 55

[PS82] Christopher C Paige e Michael A Saunders. Lsqr: An algorithm for sparse linear equations and sparse least squares. ACM Transactions on Mathematical Software (TOMS), 8(1):43-71, 1982. 18

[PWP $\left.{ }^{+} 99\right]$ Russell A Poldrack, Anthony D Wagner, Matthew W Prull, John E Desmond, Gary H Glover e John DE Gabrieli. Functional specialization for semantic and phonological processing in the left inferior prefrontal cortex. Neuroimage, 10(1):15-35, 1999. 3

[RM11] Cristina Rosazza e Ludovico Minati. Resting-state brain networks: literature review and clinical applications. Neurological Sciences, 32(5):773-785, 2011. 1,2

[RMS ${ }^{+}$01] Marcus E Raichle, Ann Mary MacLeod, Abraham Z Snyder, William J Powers, Debra A Gusnard e Gordon L Shulman. A default mode of brain function. Proceedings of the National Academy of Sciences, 98(2):676-682, 2001. 5, 9

[Row10] James B Rowe. Connectivity analysis is essential to understand neurological disorders. Frontiers in systems neuroscience, 4:144, 2010. 6

[RS10] Mikail Rubinov e Olaf Sporns. Complex network measures of brain connectivity: uses and interpretations. Neuroimage, 52(3):1059-1069, 2010. 7 
[RST65] Herbert E Rauch, CT Striebel e F Tung. Maximum likelihood estimates of linear dynamic systems. AIAA journal, 3(8):1445-1450, 1965. 13, 72

[Saa03] Yousef Saad. Iterative methods for sparse linear systems. Siam, 2003. 18

[SCKH04] Olaf Sporns, Dante R Chialvo, Marcus Kaiser e Claus C Hilgetag. Organization, development and function of complex brain networks. Trends in cognitive sciences, 8(9):418-425, 2004. 7

[SDK09] Kenneth Scerri, Michael Dewar e Visakan Kadirkamanathan. Estimation and model selection for an ide-based spatio-temporal model. Signal Processing, IEEE Transactions on, 57(2):482-492, 2009. 13

[SL08] Amir Shmuel e David A Leopold. Neuronal correlates of spontaneous fluctuations in fmri signals in monkey visual cortex: implications for functional connectivity at rest. Human brain mapping, 29(7):751-761, 2008. 6

[Smi04] Stephen M Smith. Overview of fmri analysis. The British Journal of Radiology, 77:S167-S175, 2004. 3

[Smi12] Stephen M Smith. The future of fmri connectivity. Neuroimage, 62(2):12571266, 2012. 17

[SS82] Robert H Shumway e David S Stoffer. An approach to time series smoothing and forecasting using the em algorithm. Journal of time series analysis, 3(4):253-264, 1982. 13, 45, 71, 72

[SS09] Catherine J Stoodley e Jeremy D Schmahmann. Functional topography in the human cerebellum: a meta-analysis of neuroimaging studies. Neuroimage, 44(2):489-501, 2009. 48

[Ste80] James H Steiger. Tests for comparing elements of a correlation matrix. Psychological bulletin, 87(2):245, 1980. 28

[Swe95] Wim Sweldens. Lifting scheme: a new philosophy in biorthogonal wavelet constructions. Em SPIE's 1995 International Symposium on Optical Science, Engineering, and Instrumentation, páginas 68-79. International Society for Optics and Photonics, 1995. 54

[Swe96] Wim Sweldens. The lifting scheme: A custom-design construction of biorthogonal wavelets. Applied and computational harmonic analysis, 3(2):186-200, 1996. 54

[Swe98] Wim Sweldens. The lifting scheme: A construction of second generation wavelets. SIAM Journal on Mathematical Analysis, 29(2):511-546, 1998. 54

[TAGM03] Constandinos N Theophilides, Sean C Ahearn, Sue Grady e Mario Merlino. Identifying west nile virus risk areas: The dynamic continuous-area space-time system. American Journal of Epidemiology, 157(9):843-854, 2003. 13

[TBM79] Howard L Taylor, Stephen C Banks e John F McCoy. Deconvolution with the ? 1 norm. Geophysics, 44(1):39-52, 1979. 18 
[Thi01] Henning Thielemann. Adaptive construction of wavelets for image compression. Tese de Doutorado, Master?s thesis, Martin-Luther-Universität HalleWittenberg, Fachbereich Mathematik/Informatik, 2001. 55

[Tin14] Michael Ting. Sparse image reconstruction. Molecular Imaging in Nano MRI, $1: 7-14,2014.18$

[TJ95] Hoang-Lan Nguyen Thi e Christian Jutten. Blind source separation for convolutive mixtures. Signal processing, 45(2):209-229, 1995. 57

[TSE94] Giulio Tononi, Olaf Sporns e Gerald M Edelman. A measure for brain complexity: relating functional segregation and integration in the nervous system. Proceedings of the National Academy of Sciences, 91(11):5033-5037, 1994. 7

[VAB14] Gilson Vieira, Edson Amaro e Luiz Baccala. Local sparse component analysis for blind source separation: an application to resting state fmri. Em Engineering in Medicine and Biology Society (EMBC), 2014 36th Annual International Conference of the IEEE, páginas 5611-5614. IEEE, 2014. 15, 16, 17, 49

[VAB15] Gilson Vieira, Edson Amaro e Luiz Baccala. Local dimension-reduced dynamical spatio-temporal models for resting state network estimation. Brain Informatics, 4:1-11, 2015. 15, 17, 18

[VASB15] Gilson Vieira, Edson Amaro, Joao Sato e Luiz Baccala. Independent component versus local sparse component analysis in resting state fmri. Em Engineering in Medicine and Biology Society (EMBC), 2015 37th Annual International Conference of the IEEE, páginas 511-514. IEEE, 2015. 15

[vdHMK ${ }^{+}$09] Martijn P van den Heuvel, Rene CW Mandl, Rene S Kahn, Hulshoff Pol e E Hilleke. Functionally linked resting-state networks reflect the underlying structural connectivity architecture of the human brain. Human brain mapping, 30(10):3127-3141, 2009. 9

[vdHMLP08] Martijn van den Heuvel, René Mandl, Judith Luigjes e Hilleke Hulshoff Pol. Microstructural organization of the cingulum tract and the level of default mode functional connectivity. The Journal of neuroscience, 28(43):1084410851, 2008. 9

[VDHP10] Martijn P Van Den Heuvel e Hilleke E Hulshoff Pol. Exploring the brain network: a review on resting-state fmri functional connectivity. European $\mathrm{Neu}$ ropsychopharmacology, 20(8):519-534, 2010. 1, 5, 6, 9

[vdHPH10] Martijn P van den Heuvel, Hulshoff Pol e E Hilleke. Specific somatotopic organization of functional connections of the primary motor network during resting state. Human brain mapping, 31(4):631-644, 2010. 9

[Vid99a] Brani Vidakovic. Statistical modeling by wavelets, volume 503. John Wiley \& Sons, 1999. 26

[Vid99b] Brani Vidakovic. Statistical modeling by wavelets, volume 503. John Wiley \& Sons, 1999. 66 
$\left[\mathrm{VPF}^{+} 07\right]$ JL Vincent, GH Patel, MD Fox, AZ Snyder, JT Baker, DC Van Essen, JM Zempel, LH Snyder, M Corbetta e ME Raichle. Intrinsic functional architecture in the anaesthetized monkey brain. Nature, 447(7140):83-86, 2007. 6

[VS04] Pedro A Valdes-Sosa. Spatio-temporal autoregressive models defined over brain manifolds. Neuroinformatics, 2(2):239-250, 2004. 14

[WC99] Christopher K Wikle e Noel Cressie. A dimension-reduced approach to spacetime kalman filtering. Biometrika, 86(4):815-829, 1999. 11, 13, 18

[WCLE05] Keith J Worsley, Jen-I Chen, Jason Lerch e Alan C Evans. Comparing functional connectivity via thresholding correlations and singular value decomposition. Philosophical Transactions of the Royal Society B: Biological Sciences, 360(1457):913-920, 2005. 3, 15

[WHHL12] L Wang, DF Hermens, IB Hickie e J Lagopoulos. A systematic review of resting-state functional-mri studies in major depression. Journal of affective disorders, 142(1):6-12, 2012. 1

[WJBS04] Mark W Woolrich, Mark Jenkinson, J Michael Brady e Stephen M Smith. Fully bayesian spatio-temporal modeling of fmri data. Medical Imaging, IEEE Transactions on, 23(2):213-231, 2004. 13

[WN11] Kelly P Westlake e Srikantan S Nagarajan. Functional connectivity in relation to motor performance and recovery after stroke. Frontiers in systems neuroscience, 5:8, 2011. 6

[WNF09] Stephen J Wright, Robert D Nowak e Mário AT Figueiredo. Sparse reconstruction by separable approximation. Signal Processing, IEEE Transactions on, 57(7):2479-2493, 2009. 23

[Wu83] CF Jeff Wu. On the convergence properties of the em algorithm. The Annals of statistics, 1:95-103, 1983. 15

[WZC13] Nizhuan Wang, Weiming Zeng e Lei Chen. Sacica: A sparse approximation coefficient-based ica model for functional magnetic resonance imaging data analysis. Journal of neuroscience methods, 216(1):49-61, 2013. 17

[WZH10] Jinhui Wang, Xinian Zuo e Yong He. Graph-based network analysis of restingstate functional mri. Frontiers in systems neuroscience, 4:16, 2010. 3, 50

$\left[\mathrm{XMW}^{+}\right.$09] Jinhu Xiong, Liangsuo Ma, Binquan Wang, Shalini Narayana, Eugene P Duff, Gary F Egan e Peter T Fox. Long-term motor training induced changes in regional cerebral blood flow in both task and resting states. Neuroimage, 45(1):75-82, 2009. 9

$\left[\mathrm{ZP}^{+} 01\right]$ Michael Zibulevsky, Barak Pearlmutter et al. Blind source separation by sparse decomposition in a signal dictionary. Neural computation, 13(4):863-882, 2001. 10, 16, 18, 60, 62 